\title{
1 Genome analyses reveal the hybrid origin of the staple food crop white Guinea yam
}

4 Yu Sugihara ${ }^{1}$, Kwabena Darkwa $^{2}$, Hiroki Yaegashi ${ }^{3}$, Satoshi Natsume ${ }^{3}$, Motoki Shimizu ${ }^{3}$, Akira Abe ${ }^{3}$,

5 Akiko Hirabuchi ${ }^{3}$, Kazue Ito $^{3}$, Kaori Oikawa ${ }^{3}$, Muluneh Tamiru-Oli ${ }^{34}$, Atsushi Ohta ${ }^{1}$, Ryo

6 Matsumoto $^{2}$, Agre Paterne ${ }^{2}$, David De Koeyer ${ }^{25}$, Babil Pachakkil ${ }^{67}$, Shinsuke Yamanaka ${ }^{6}$, Satoru

7 Muranaka $^{6}$, Hiroko Takagi ${ }^{6}$, Ben White $^{8}$, Robert Asiedu ${ }^{2}$, Hideki Innan ${ }^{9}$, Asrat Asfaw ${ }^{2 *}$, Patrick

8 Adebola $^{2^{*}}$, Ryohei Terauchi ${ }^{13 *}$

$10{ }^{1}$ Laboratory of Crop Evolution, Graduate School of Agriculture, Kyoto University, Kyoto, Japan

$11{ }^{2}$ International Institute of Tropical Agriculture (IITA), Ibadan, Nigeria

$12{ }^{3}$ Iwate Biotechnology Research Center, Kitakami, Iwate, Japan

$13{ }^{4}$ Department of Animal, Plant and Soil Sciences, School of Life Sciences, AgriBio Building, La Trobe

14 University, Melbourne, Australia

$15{ }^{5}$ Fredericton Research and Development Centre, Agriculture and Agri-Food Canada, Fredericton, New

16 Brunswick, Canada

$18{ }^{6}$ Japan International Research Center for Agricultural Sciences, Tsukuba, Japan

$19{ }^{7}$ Department of International Agricultural Development, Tokyo University of Agriculture, Tokyo, Japan.

${ }^{8}$ Earlham Institute, Norwich, UK

${ }^{9}$ Laboratory of Population Genetics and Genome Evolution, The Graduate University for Advanced Studies,

* Co-corresponding authors: 
Abstract

White Guinea yam (Dioscorea rotundata) is an important staple tuber crop of West Africa. However, its origin remains unclear. In this study, we re-sequenced 336 accessions of white Guinea yam and compared them with the sequences of the wild Dioscorea species using an improved reference genome sequence of $D$. rotundata. Our results suggest a hybrid origin of white Guinea yam from crosses between the rainforest wild species D. praehensilis and the savannah-adapted D. abyssinica. We identified a higher genomic contribution from $D$. abyssinica in the sex chromosome of Guinea yam and an extensive introgression around the SWEETIE gene. Our findings point to a complex domestication scenario for Guinea yam and highlight the importance of wild species as gene donors for improvement of this crop through molecular breeding.

Introduction

Yams (Dioscorea spp.) are major starchy tuber crops in the tropics. Overall, ten yam species are cultivated around the world, including D. alata in Southeast Asia, D. trifida in South America, and D. rotundata in West and Central Africa (1). D. rotundata also known as white Guinea yam is the most important species in West and Central Africa, an area accounting for $92.5 \%$ of the global yam production in 2018 (http://www.fao.org/statistics). Beyond its nutritional and food values, Guinea yam is also important for the culture of West African people (2). Recently, a whole genome sequence of Guinea yam was reported (3).

Despite the considerable importance of Guinea yam, its origin has been elusive. Two types of Guinea yams are known; white Guinea yam (D. rotundata) and yellow Guinea yam (D. cayenensis). D. cayenensis was proposed to be a triploid species of hybrid origin with D. rotundata and $D$. burkilliana as the maternal and paternal parent, respectively $(4,5)$. It was also suggested that the 
triploid D. rotundata is a hybrid between D. rotundata and D. togoensis (5). However, the origin of diploid D. rotundata, which represents the majority of Guinea yam, has been ambiguous. There are two candidate wild species as the progenitors of diploid D. rotundata; a savannah-adapted wild species $D$. abyssinica and a rainforest-adapted wild species $D$. praehensilis. A recent genome study involving 86 D. rotundata, 47 D. praehensilis and 34 D. abyssinica accessions proposed that diploid D. rotundata was domesticated from D. praehensilis (6). Here, we address this hypothesis using an expanded set of wild and cultivated Dioscorea genomes.

In this study, we generated an improved version of the Guinea yam reference genome, and used it to analyze the genomes of 336 accessions of $D$. rotundata and its wild relatives. Based on these analyses, we attempted to reveal the history of Guinea yam domestication. Our results suggest that diploid $D$. rotundata was most likely derived from homoploid hybridization between $D$. abyssinica and D. praehensilis. By evaluating the genomic contributions of each parental species to $D$. rotundata, we revealed a higher representation of $D$. abyssinica genome in the sex chromosome and a signature of extensive introgression in SWEETIE gene on chromosome 17.

\section{Genetic diversity of Guinea yam}

We obtained DNA samples of 336 accessions of D. rotundata maintained at IITA, Nigeria, representing the genetic diversity of Guinea yam landraces and improved lines of West Africa. These samples were subjected to whole genome resequencing by illumina sequencing platform. The resulting short reads were aligned to the newly assembled reference genome (supplementary text S1 and S2) and SNP information was extracted to use for genetic diversity studies (supplementary text S3). Based on admixture analysis by $\operatorname{sNMF}(7)$, we defined five major clusters (Fig. 1A). When $K$ is 2, cluster 1 was clearly separated from the other accessions. Principal Component Analysis (PCA) also separated cluster 1 from the rest (Fig. 1B). Accessions in cluster 1 had a higher heterozygosity and $\sim 10$ times larger number of unique alleles than those in the remaining four clusters (Fig. S1 and 
Fig. S2). Because flow cytometry analysis confirmed that all 10 accessions analyzed in cluster 1 were triploids (Table S1), we hypothesized that cluster 1 represents triploid D. rotundata that was reported as a hybrid between $D$. rotundata and D. togoensis (5). After removing the cluster 1 accessions, nucleotide diversity of $D$. rotundata was estimated to be $14.83 \times 10^{-4}$ (Table S2), which is approximately 1.5 times larger than that reported previously (6).

\section{Phylogenomic analysis of African yam}

Using the SNP information, we constructed a rooted Neighbor-joining (NJ) tree (8) based on 308 Guinea yam accessions sequenced in the present study excluding cluster 1 triploid accessions, as well as 80 D. rotundata, 29 D. abysinica, 21 Western D. praehensilis, and 18 Cameroonian D. praehensilis as sequenced in the previous study ( 6 ) using two accessions of Asian species D. alata as an outgroup (Fig. 1C). According to this NJ tree, D. rotundata accessions sequenced in this study were genetically close to the $D$. rotundata accessions reported in the previous study (6) (Fig. 1C). However, the $\mathrm{NJ}$ tree showed that $D$. rotundata was more closely related to D. abyssinica than to Western D. praehensilis (Fig. 1C), which is inconsistent with the previous report (6) showing that $D$. rotundata was most closely related to Western $D$. praehensilis.

To elucidate the evolutionary relationships of the three wild Dioscorea species, D. abyssinica (indicated as A), Western D. praehensilis (P). Cameroonian D. praehensilis (C) that are closely related to $D$. rotundata, we adopted the $\partial \mathrm{a} \partial \mathrm{i}$ analysis (9), which allows estimating demographic parameters from an unfolded site frequency spectrum. First, three phylogenetic models, $\{\{A, P\}, C\}$, $\{\{\mathrm{C}, \mathrm{P}\}, \mathrm{A}\},\{\{\mathrm{C}, \mathrm{A}\}, \mathrm{P}\}$ were tested using 17,532 SNPs that were polarized using an outgroup $D$. alata without considering migration among the species. Out of the three models, $\{\{\mathrm{A}, \mathrm{P}\}, \mathrm{C}\}$ had the highest likelihood (Table. S3). This result is not consistent with the previous study $(6)$ where $\{\{\mathrm{P}$, C \}, A \} had the highest likelihood as studied by a different method with fastsimcoal2 (10). To exactly repeat the previous analysis, we tested these three models with fastsimcoal2 (10) on the previous 
reference genome (3), resulting in $\{\{\mathrm{A}, \mathrm{P}\}, \mathrm{C}\}$ with the highest likelihood (Table S4). Taken together, our result is not consistent with the previous report (6). However, it is consistent with the PCA result of the same report, where Cameroonian D. praehensilis is separated from the other African yams in the PC1 (Fig. 2A of (6)). Based on the assumption that $\{\{\mathrm{A}, \mathrm{P}\}, \mathrm{C}\}$ is the true evolutionary relationship among the three wild Dioscorea species, the evolutionary parameters were re-estimated by $\partial \mathrm{a} \partial \mathrm{i}$ allowing symmetric migration among the species (Fig. 1D). Since our result shows that Cameroonian $D$. praehensilis is distantly related to D. rotundata and is unlikely involved in genetic exchange with D. rotundata (Fig. 1C), we hereafter focus on Western D. praehensislis and designate it as $D$. praehensilis for brevity.

\section{Hybrid origin of Guinea yam}

Three hypotheses of the origin of Guinea yam (D. rotundata) can be proposed from the results of NJ tree (Fig. 1C) and $\partial \mathrm{a} \partial \mathrm{i}$ (9) (Fig. 1D). The first is that D. rotundata was derived from D. abyssinica (Hypothesis 1 in Fig. 2A). The second is that D. rotundata was derived from D. praehensilis (Hypothesis 2 in Fig. 2A). However, in Hypotheses 1 and 2, the divergent time of D. rotundata from the wild species may not be sufficient to separate the three lineages and there is incomplete lineage sorting among them. The third hypothesis is that D. rotundata was originated as an admixture between D. abyssinica and D. praehensilis (Hypothesis 3 in Fig. 2A).

Before estimating the evolutionary parameters for the three hypotheses, we studied the allele frequencies of 388 D. rotundata sequences including 80 in the previous study (6) focusing on 144 SNPs that are positioned over the entire genome and are oppositely fixed in the two candidate progenitors (Fig. 2B). If Hypothesis 1 or 2 is correct, allele frequencies in these 144 SNPs should be highly skewed to either of the progenitors. However, the patterns of allele contribution from the two candidate species to $D$. rotundata is almost same. This result suggests that the admixture origin of Guinea yam (Hypothesis 3) is most likely. 
The three hypotheses were tested by $\partial \mathrm{a} \partial \mathrm{i}(9)$ with symmetric migration rates, using $15,461 \mathrm{SNPs}$ polarized by D. alata (Fig. 2A), which showed that Hypothesis 3 had the highest likelihood and the lowest Akaike information criterion (AIC) (Fig. 2A and Table. S3). This result is in support of the admixture hypothesis that $D$. rotundata was derived from crosses between $D$. abyssinica and $D$. praehensilis. The estimated parameters by $\partial \mathrm{a} \partial \mathrm{i}$ indicates that the hybridization between $D$. abyssinica and D. praehensilis was relatively recent in relation to the divergence between the two wild species, and it also indicates that the genomic contribution from D. abyssinica and that from D. praehensilis were approximately $68 \%$ and $32 \%$, respectively. Introgression generally results in highly asymmetric genomic contributions from the parental species, whereas hybridization shows symmetric genomic contributions (11). The observed intermediate genomic contributions support the hybridization rather than the introgression hypothesis.

To evaluate the genetic distances of $D$. rotundata from the two parental species for each chromosome, $F_{S T}(12)$ was calculated (Fig. 2D). We observed varying genetic distances from the two parents across the different chromosomes, while the overall genetic distance of D. rotundata from $D$. abyssinica was smaller than that from D. praehensilis (Fig. 2D). Intriguingly, chromosome 11, to which we previously mapped the candidate locus for sex determination (3), had the shortest genetic distance from $D$. abyssinica and the longest genetic distance from $D$. praehensilis among the all chromosomes, indicating that chromosome 11 of $D$. rotundata is highly skewed to D. abyssinica (Fig. 2D). This observation mirrors the case of the X chromosome in Anopheles gambiae complex (African mosquito) (13).

\section{Evolutionary history of Guinea yam}

To infer the maternal history of Guinea yam, a haplotype network of the whole plastid genome was constructed using all samples used in the NJ tree (Fig. 1C) as well as the triploid accessions in cluster 
162

163

164

165

praehensilis has the largest genetic distance from $D$. rotundata. This result is in line with the phylogenomic trees of African yam (Fig. 1C and Fig. 1D). Strikingly, plastid genomes of diploid and triploid D. rotundata are uniform, and are very similar to that of Nigerian or Beninese D. abyssinica. Plastid genomes of D. praehensilis from Nigeria, Benin and Ghana seem derived from Nigerian or Beninese $D$. abyssinica. These results indicate that $D$. abyssinica is an older lineage than $D$. praehensilis and that the place of origins of D. rotundata and D. praehensilis is probably around Nigeria or Benin. Using whole genome diversity of $D$. rotundata, a recent study ( 6 ) has hypothesized that the origin of $D$. rotundata was around north Benin, and our result supports this. Plastid genomes of some wild species are identical to those of cultivated Guinea yams. Gene flow from cultivated yams to wild yams may account for this observation (14).

The results of nuclear genome admixture (Fig. 2) and plastid haplotype network (Fig. 3A) indicate that the maternal origin of diploid D. rotundata was $D$. abysinica and its paternal origin was $D$. praehensilis (Fig. 3B). Hybridization between D. abyssinica and D. praehensilis has been reported to be rare (15), but such rare hybrids seem to have been domesticated by humans. The triploid $D$. rotundata shared the plastid haplotype with diploid D. rotundata, therefore diploid D. rotundata served as the maternal parent and D. togoensis as the paternal parent. D. cayenensis is reported to have D. rotundata as the maternal parent and D. burkilliana as the paternal parent $(4,5)$. All cultivated Guinea yams are hybrids with $D$. abyssinica plastid genomes.

To understand the change of population sizes, demographic history of African yam was re-inferred by $\partial \mathrm{a} \partial \mathrm{i}(9)$ allowing migration (Fig. 3C and supplementary text S7). The same dataset to Fig. 2C was used for this analysis. By fixing the parameters predicted in Fig. $2 \mathrm{C}$ except for the population sizes, we re-estimated each population size at the start and end points after the emergence of those species assuming an exponential increase/decrease of the population sizes. According to this analysis, after the emergence of the wild progenitors of Guinea yam, the population size of D. abyssinica is decreasing, while that of $D$. praehensilis is increasing (Fig. 3C). This finding may indicate $D$. 
praehensilis population was possibly derived from $D$. abyssinica, which is consistent with the result of the haplotype network (Fig. 3A).

\section{Extensive introgression at the SWEETIE locus}

To explore multiple introgression to $D$. rotundata from the two wild species, the $f_{4}$ statistic (16) was analyzed using the four groups: a) D. rotundata cluster 2 and 5, b) D. rotundata cluster 4 and c) D. abyssinica and d) D. praehensilis (supplementary text S8). $f_{4}$ statistic reveals the representation of two alternative discordant genealogies (Fig. 4A). Basically, $f_{4}$ value is close to zero if the two groups (group a and b) of $D$. rotundata show a concordant genealogy in relation to D. abyssinica and $D$. praehensilis. On the other hand, if the two groups of $D$. rotundata exhibit discordant genealogy and a large genetic distance to each other, $f_{4}$ is diverged from zero. We obtained $f_{4}$ statistic, $f_{4}\left(P_{25}, P_{4}, P_{\mathrm{P}}\right.$, $P_{\mathrm{A}}$ ) for each SNP and applied a sliding window analysis (Fig. 4B). $f_{4}$ value was close to zero across the genome indicating that overall we cannot decide between topology 1 and 2 . However, the genomic regions around the SWEETIE gene showed the lowest $f_{4}\left(P_{25}, P_{4}, P_{\mathrm{P}}, P_{\mathrm{A}}\right)\left[Z\left(f_{4}\right)=-5.66\right]$, with overrepresentation of topology 2 in the SWEETIE gene (DRNTG_01731). To see the genealogical relationships around the SWEETIE gene, Neighbor-Net (17) was constructed around that locus (4.00 Mbp $~ 4.15 \mathrm{Mbp}$ on chromosome 17) (Fig. 4C). Neighbor-Net showed that the locus of cluster 4 was close to that of $D$. praehensilis, while those of cluster 2 and 5 and some other accessions were close to D. abyssinica. This indicates that the SWEETIE gene was introgressed from the wild species more than one time. The SWEETIE gene encodes a membrane protein that is known to be involved in general control of sugar flux (18). In Arabidopsis, the sweetie mutant shows pronounced changes in the accumulation of sugar, starch and ethylene with significant growth and developmental alterations (19). We still do not know the effect of this introgression on the phenotype of Guinea yam, but this locus seems to be a target of selection. 
213 Homoploid hybridization can contribute to increased genetic variation by recombination between

214 distantly related species, and it often allows the hybrid to adapt to unexploited niches (20). In the case 215 of Guinea yam, the savannah-adapted wild species $D$. abyssinica and the rainforest-adapted wild 216 species D. praehensilis have not been suitable for agriculture; however, their hybrid D. rotundata 217 could have been adopted by humans to the man-made environment. Gene combinations from different 218 wild yams might have contributed to the Guinea yam domestication. New alleles from wild yams 219 seems to have been introduced to cultivated Guinea yams like the SWEETIE gene, and it probably conferred plants with beneficial phenotypes for humans. This study highlights the need to consider how to effectively leverage gene pools of wild species from different habitat for rapid breeding of Guinea yam using genomics information.

\section{Acknowledgements}

This study has been carried out under AfricaYam Project funded by Bill and Melinda Gates Foundation (BMGF) as well as EDITS-Yam project funded by JIRCAS, Japan. The authors thank Sophien Kamoun for valuable comments in the preparation of manuscript.

\section{Reference and Notes}

1. J. F. Hancock, "Starch Staples and Sugar" in Plant evolution and the origin of crop species (CABI Publishing, Wallingford, UK, ed. 3, 2012).

2. J. E. Obidiegwu, E. M. Akpabio, The geography of yam cultivation in southern Nigeria: Exploring its social meanings and cultural functions. J. Ethnic. Foods. 4, 28-35 (2017).

3. M. Tamiru, S. Natsume, H. Takagi, B. White, H. Yaegashi, M. Shimizu, K. Yoshida, A. Uemura, K. Oikawa, A. Abe, N. Urasaki, H. Matsumura, P. Babil, S. Yamanaka, R. Matsumoto, S. 
I. Rabbi, M. Tsujimura, T. Terachi, W. Haerty, M. Corpas, S. Kamoun, G. Kahl, H. Takagi, R. Asiedu, R. Terauchi, Genome sequencing of the staple food crop white Guinea yam enables the development of a molecular marker for sex determination. BMC Biol. 15, 86 (2017).

4. R. Terauchi, V. A. Chikaleke, G. Thottappilly, S. K. Hahn, Origin and phylogeny of Guinea yams as revealed by RFLP analysis of chloroplast DNA and nuclear ribosomal DNA. Theor. Appl. Genet. 83, 743-751 (1992).

5. G. Girma, K. E. Hyma, R. Asiedu, S. E. Mitchell, M. Gedil, C. Spillane, Next-generation sequencing based genotyping, cytometry and phenotyping for understanding diversity and evolution of guinea yams. Theor. Appl. Genet. 127, 1783-1794 (2014).

6. N. Scarcelli, P. Cubry, R. Akakpo, A.-C. Thuillet, J. Obidiegwu, M. N. Baco, E. Otoo, B. Sonké, A. Dansi, G. Djedatin, C. Mariac, M. Couderc, S. Causse, K. Alix, H. Chaïr, O.

7. E. Frichot, F. Mathieu, T. Trouillon, G. Bouchard, O. François, Fast and Efficient Estimation of Individual Ancestry Coefficients. Genetics. 196, 973-983 (2014).

8. N. Saitou, M Nei, The neighbor-joining method: a new method for reconstructing phylogenetic trees. Mol. Biol. Evol. 4, 406-425 (1987).

9. R. N. Gutenkunst, R. D. Hernandez, S. H. Williamson, C. D. Bustamante, Inferring the Joint Demographic History of Multiple Populations from Multidimensional SNP Frequency Data. PLoS Genet. 5, e1000695 (2009).

10. L. Excoffier, I. Dupanloup, E. Huerta-Sánchez, V. C. Sousa, M. Foll, Robust demographic Inference from genomic and SNP data. PLoS Genet. 9, e1003905 (2013).

11. R. A. Folk, P. S. Soltis, D. E. Soltis, R. Guralnick, New prospects in the detection and comparative analysis of hybridization in the tree of life. Am. J. Bot. 105, 364-375 (2018).

12. S. Wright, The genetical structure of populations. Ann. Eugen. 15, 323-354 (1951). 
13. M. C. Fontaine, J. B. Pease, A. Steele, R. M. Waterhouse, D. E. Neafsey, I. V. Sharakhov, X. Jiang, A. B. Hall, F. Catteruccia, E. Kakani, S. N. Mitchell, Y.-C. Wu, H. A. Smith, R. R. Love, M. K. Lawniczak, M. A. Slotman, S. J. Emrich, M. W. Hahn, N. J. Besansky. Extensive introgression in a malaria vector species complex revealed by phylogenomics. Science. $\mathbf{3 4 7}$, 1258524 (2015).

14. N. Scarcelli, H. Chaïr, S. Causse, R. Vesta, T. L. Couvreur, Y. Vigouroux, Crop wild relative conservation: Wild yams are not that wild. Biol. Conserv. 210, 325-333 (2017).

15. N. Scarcelli, S. Tostain, Y. Vigouroux, C. Agbangla, O. Daïnou, J.-L. Pham, Farmers' use of wild relative and sexual reproduction in a vegetatively propagated crop. The case of yam in Benin. Mol. Ecol. 15, 2421-2431 (2006).

16. D. Reich, K. Thangaraj, N. Patterson, A. L. Price, L. Singh, Reconstructing Indian population history. Nature. 461, 489-494 (2009).

17. D. H. Huson, D. Bryant, Application of Phylogenetic Networks in Evolutionary Studies. Mol. Biol. Evol. 23, 254-267 (2006).

18. N. Veyres, M. Aono, B. S. Sangwan-Norree, R. S. Sangwan, Has Arabidopsis SWEETIE protein a role in sugar flux and utilization? Plant Signal. Behav. 3, 722-725 (2008).

19. N. Veyres, A. Danon, M. Aono, S. Galliot, Y. B. Karibasappa, A. Diet, F. O. Grandmottet, M. Arabidopsis sweetie mutant is affected in carbohydrate metabolism and defective in the control of growth, development and senescence. Plant J. 55, 665-686 (2008). 
A

C

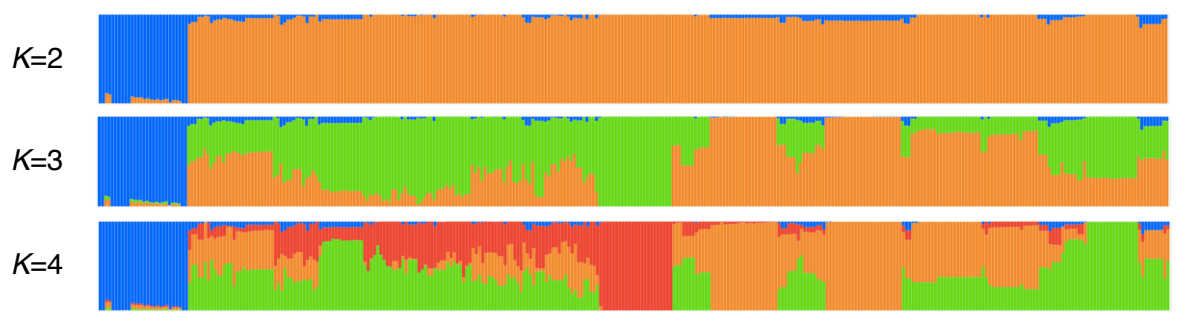

$k=5$

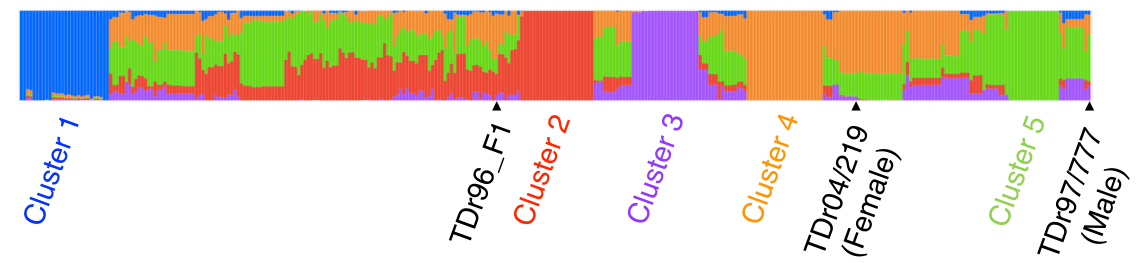

D. rotundata (in this study)

$X$ D. rotundata (in Scarcelli et al. 2019)

$\diamond D$. abyssinica
B

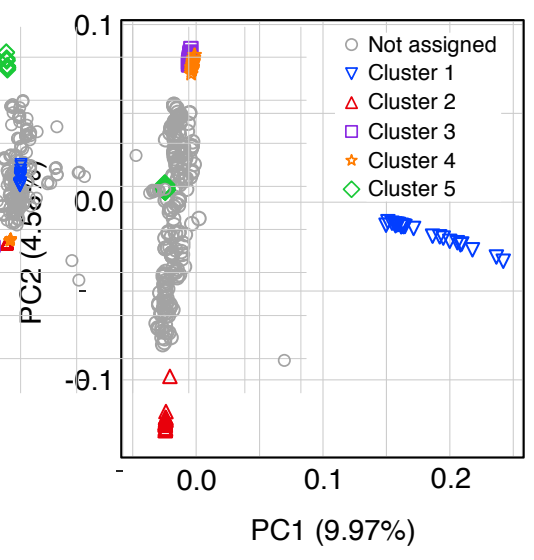

D

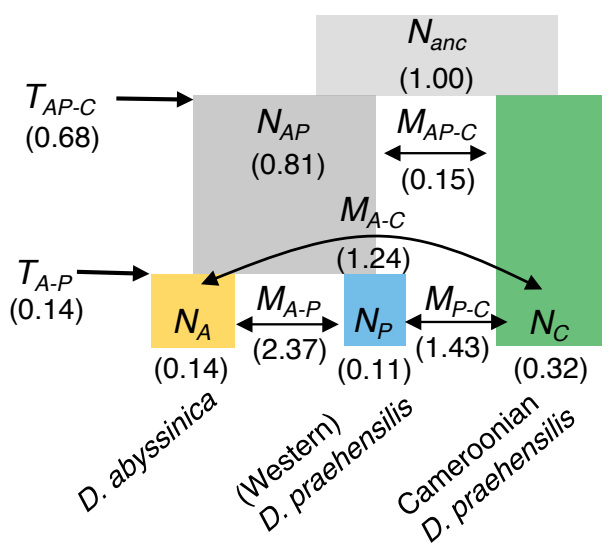

Fig. 1. Genetic diversity and phylogenomics of Guinea yam and its wild relatives. (A) Ancestry proportions of each Guinea yam accession with 6,124,093 SNPs. "TDr96_F1" is the sample used as the reference genome. (B) PCA result of the 336 Guinea yam accessions. (C) Neighbor-joining tree of four African yam lineages reconstructed using $D$. alata as an outgroup based on 463,293 SNPs. The sequences of $D$. rotundata in the previous study (6) were included in the tree as represented by "X". The 308 D. rotundata (excluding 28 accessions in cluster 1 due to the triploid accessions) analyzed in this study are close to those in the previous study (6). (D) Evolutionary relationship of three African wild yam lineages $(D$. abyssinica, Western $D$. praehensilis, Cameroonian D. praehensilis) as inferred by dadi (9) using 17,532 SNPs. $N, M$, and $T$ represent the relative population size from $N_{\text {anc }}$, migration rate, and divergence time, respectively. 
A

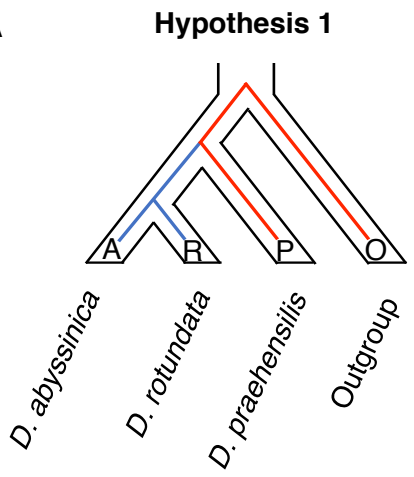

$\mathrm{AIC}=20319$
Hypothesis 2
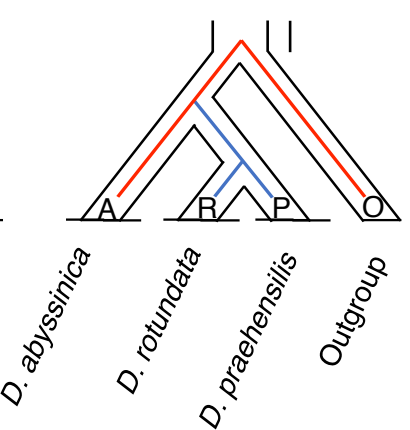

$\mathrm{AIC}=20791$

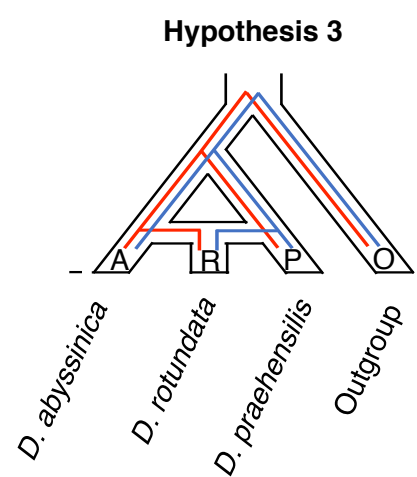

$\mathrm{AIC}=20124$

B

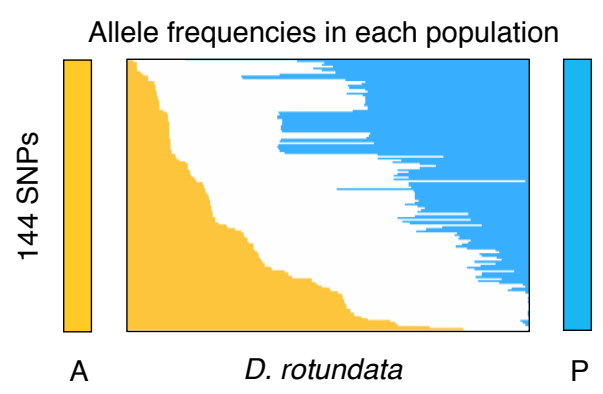

C

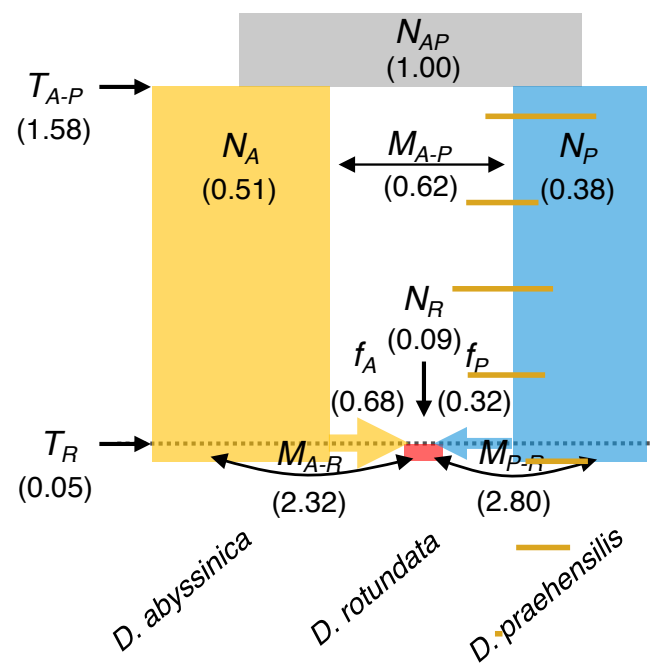

D

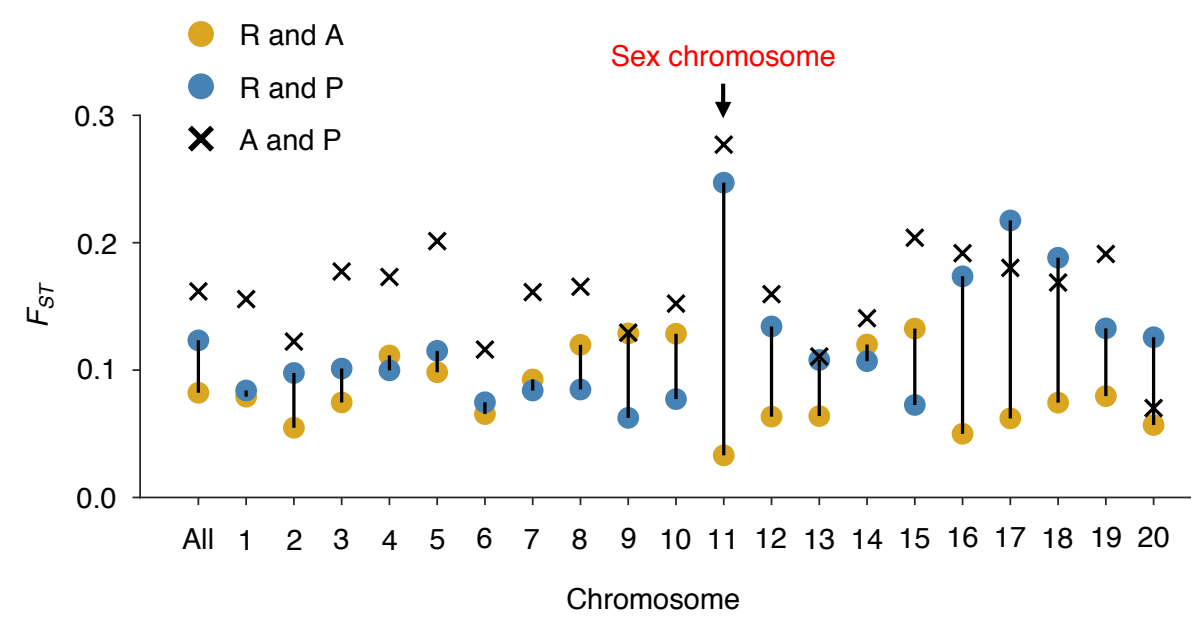

Fig. 2. Evidence for the hybrid origin of Guinea yam. (A) Hypotheses for the domestication of Guinea yam ( $D$. rotundata). Hypothesis 1 assumes that $D$. rotundata was diverged from $D$. abyssinica. Hypothesis 2 assumes that $D$. rotundata was diverged from $D$. praehensilis. Hypothesis 3 assumes that $D$. rotundata was derived from the hybrid between $D$. abyssinica and $D$. praehensilis. D. alata was used as an outgroup. (B) Frequencies of fixed alleles of $D$. abyssinica $(\mathrm{A})$ and $D$. praehensilis $(\mathrm{P})$ among the $388 D$. rotundata sequences including 80 in the previous study (6). (C) Evolutionary parameters related to the hybrid origin of Guinea yam as inferred by dadi (9) using 15,461 SNPs. (D) $F_{S T}$ among the three African yams, D. rotundata (R), D. abyssinica (A) and $D$. praehensilis $(\mathrm{P})$ for each chromosome. Chromosome 11 of $D$. rotundata containing sex locus shows a lower distance to that of $D$. abyssinica. 
A

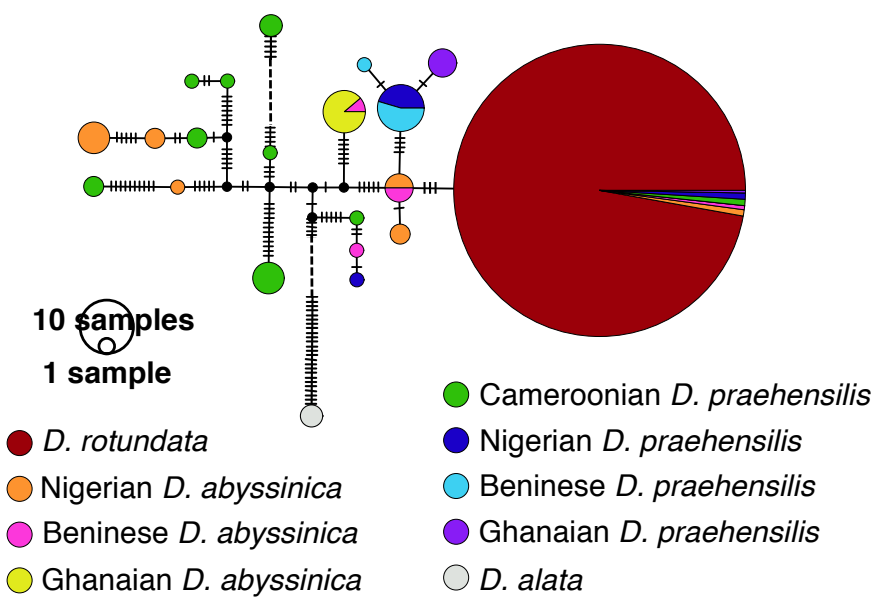

C

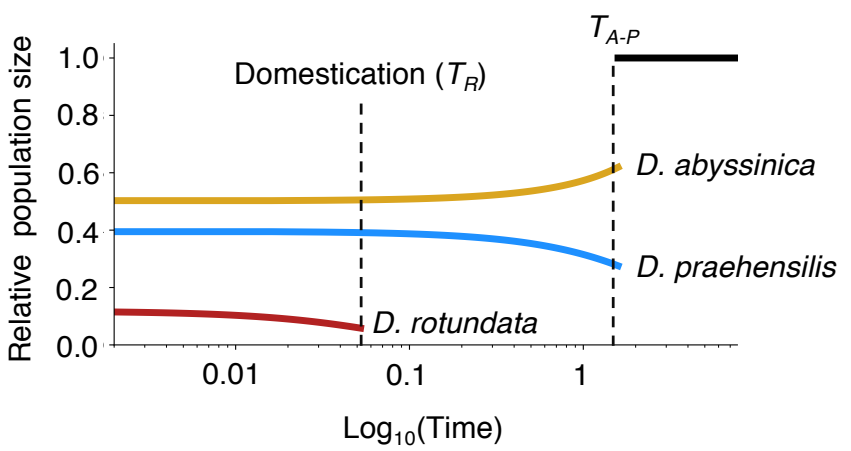

B

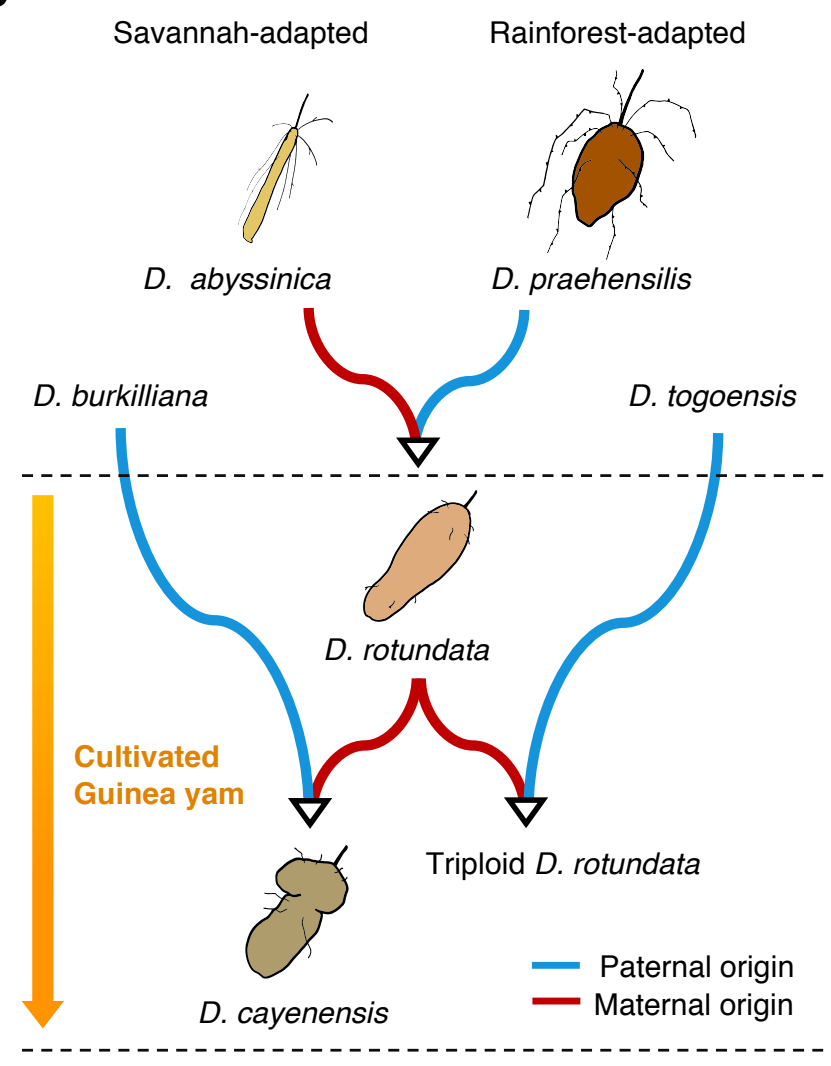

Fig. 3. Evolutionary scenario of African yam origins. (A) Haplotype network of the whole plastid genomes of $416 D$. rotundata (including the triploid accessions), 68 wild relatives, and two $D$. alata used as the outgroup. The number of vertical dashes represent the number of mutations. Western (Nigerian, Beninese, and Ghanaian) $D$. praehensilis and $D$. rotundata seem diverged from Nigerian and Beninese $D$. abyssinica. (B) Domestication process of Guinea yam. The blue line represents the paternal origin, and the red line represents the maternal origin. (C) Changes of population sizes of $D$. rotundata and its wild relatives as inferred by dadi (9). The parameters except for that of population size were identical to those used in Fig. 2C. After the domestication of $D$. rotundata, the population size of $D$. rotundata has been increasing with the migration from the wild progenitors. 
A
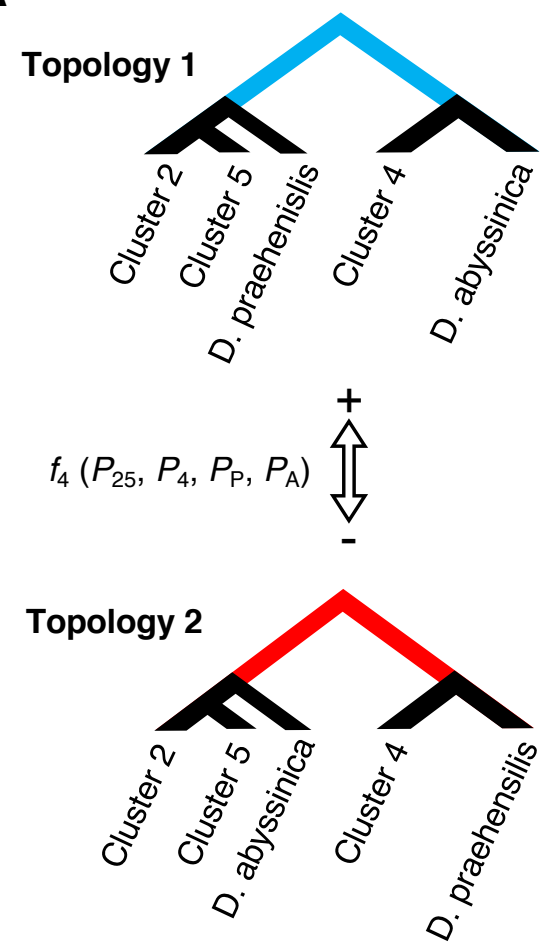

C

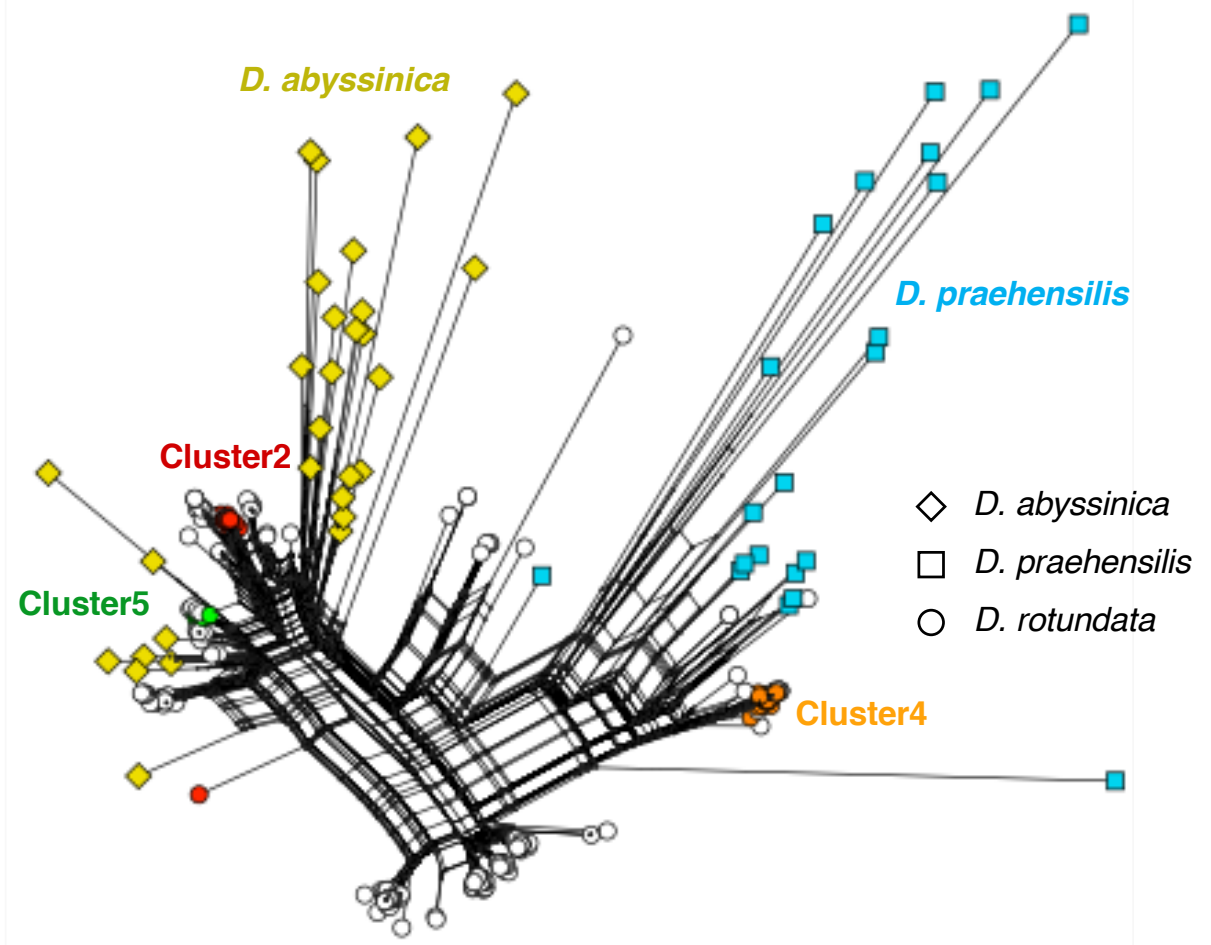

B

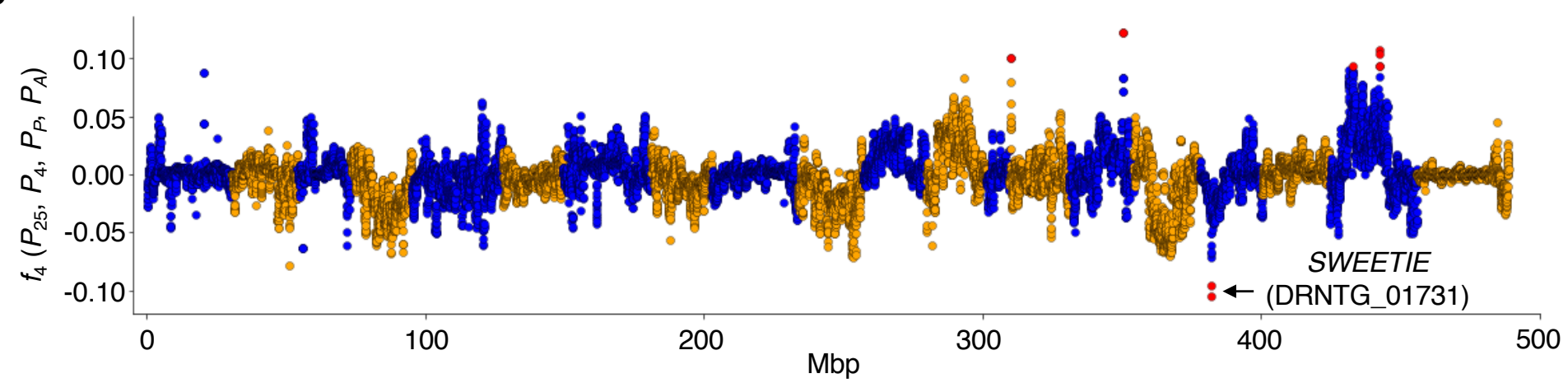

Fig. 4. Signature of extensive introgression around the SWEETIE gene. (A) Topology of $f_{4}\left(P_{25}, P_{4}, P_{P}, P_{A}\right)$ in cluster 2, 4, 5 and wild yams. Positive $f_{4}$ values represent the long internal branch of the upper tree (Topology 1). Negative $f_{4}$ values represent the long internal branch of the bottom tree (Topology 2). (B) $f_{4}$ values across the genome. This was conducted with $250 \mathrm{~Kb}$ window and $25 \mathrm{~Kb}$ step. Red dots indicates outliers of the sliding window which have $\left|Z\left(f_{4}\right)\right|>5$. The locus around the SWEETIE gene shows extraordinarily negative $f_{4}$ values. (C) Neighbor-Net around the SWEETIE gene (4Mbp $\sim 4.15 \mathrm{Mbp}$ on chromosome 17). This was constructed by SplitsTree (17) using 458 variants. 


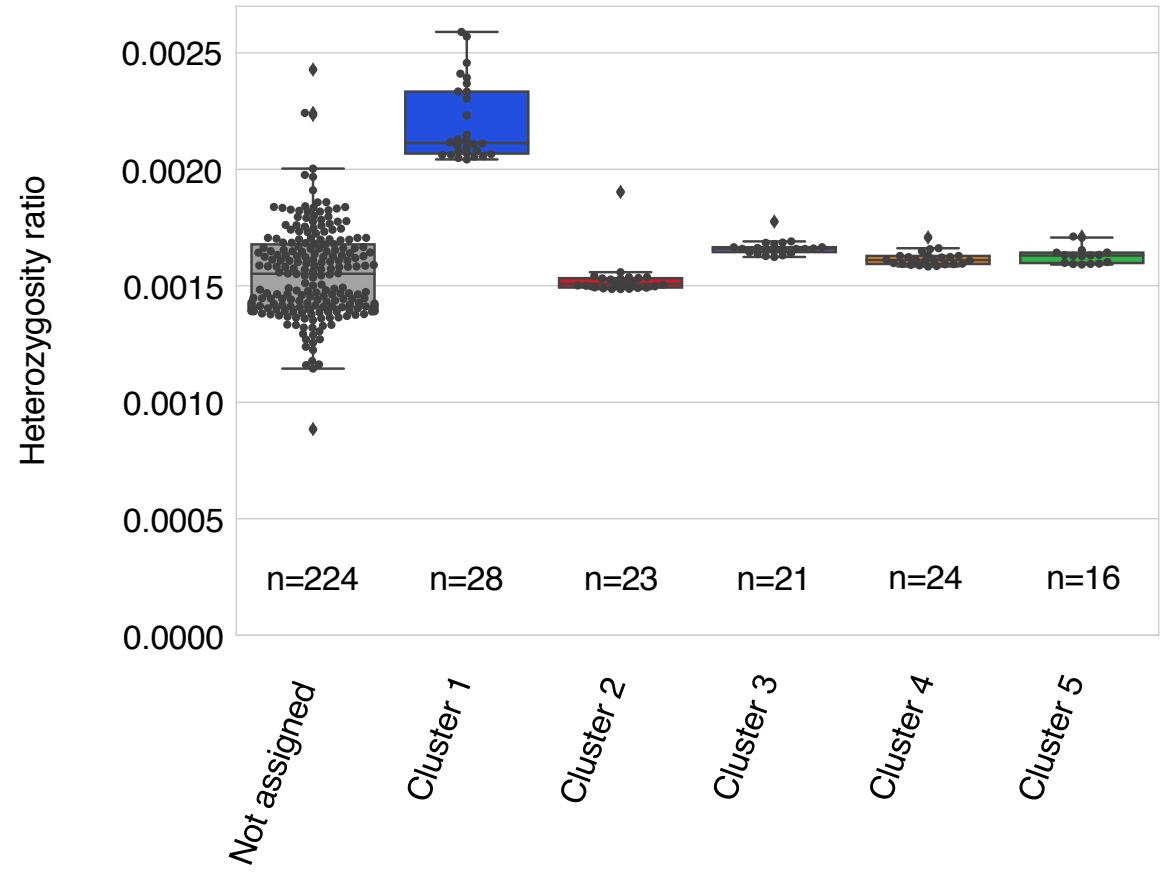

Fig. S1. Heterozygosity ratio in five clusters of $D$. rotundata.

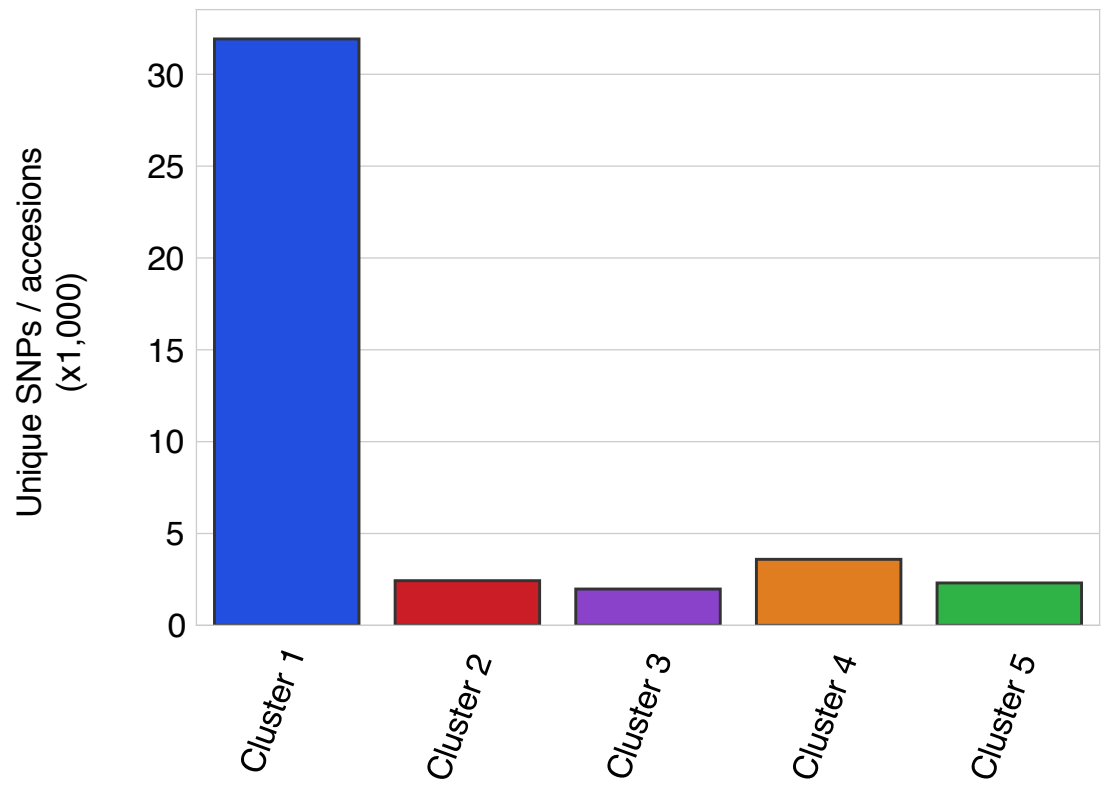

Fig. S2. Number of unique alleles in the five clusters of $D$. rotundata. 
Table S2. Population genetics summary statistic in the $\mathbf{3 0 8}$ yam accessions

After imputation

No. segregating site

$5,229,368$

No. singleton

$1,227,900$

$\theta_{W}$

$14.98 \times 10^{-4}$

$\theta_{\pi}$

$14.83 \times 10^{-4}$

Tajima's $D$

$-0.0305$

Table S3. Likelihood comparison in dadi

\begin{tabular}{|c|c|c|c|c|}
\hline Model & $\log _{10}(L)$ & $\begin{array}{r}\text { No. } \\
\text { parameters }\end{array}$ & AIC & $\begin{array}{r}\text { Illustration of } \\
\text { the model }\end{array}$ \\
\hline $\begin{array}{l}\{\{\mathrm{A}, \mathrm{P}\}, \mathrm{C}\} \\
\text { (without migration) }\end{array}$ & -15289.70 & 6 & 30591.40 & - \\
\hline $\begin{array}{l}\{\{\mathrm{C}, \mathrm{P}\}, \mathrm{A}\} \\
\text { (without migration) }\end{array}$ & -15765.32 & 6 & 31542.64 & - \\
\hline $\begin{array}{l}\{\{\mathrm{C}, \mathrm{A}\}, \mathrm{P}\} \\
\text { (without migration) }\end{array}$ & -15765.15 & 6 & 31542.29 & - \\
\hline $\begin{array}{l}\{\{\mathrm{A}, \mathrm{P}\}, \mathrm{C}\} \\
\text { (with migration) }\end{array}$ & -12739.86 & 10 & 25499.72 & Fig. 1D \\
\hline $\begin{array}{l}\{\{\mathrm{A}, \mathrm{R}\}, \mathrm{P}\} \\
\text { (with migration) }\end{array}$ & -10149.73 & 10 & 20319.47 & - \\
\hline $\begin{array}{l}\{\{\mathrm{P}, \mathrm{R}\}, \mathrm{A}\} \\
\text { (with migration) }\end{array}$ & -10385.46 & 10 & 20790.92 & - \\
\hline $\begin{array}{l}\{\{\mathrm{A}, \mathrm{R}\},\{\mathrm{P}, \mathrm{R}\}\} \\
\text { (with migration) }\end{array}$ & -10052.96 & 9 & 20123.91 & Fig. 2C \\
\hline $\begin{array}{ll}\{\{\mathrm{A}, \mathrm{R}\},\{\mathrm{P}, \mathrm{R}\}\} \\
-\quad \text { With migration } \\
-\quad \text { With population growth } \\
-\quad \text { Fix the parameters except } \\
\quad \text { for population size }\end{array}$ & -10046.73 & 6 & 20105.47 & Fig. 3C \\
\hline
\end{tabular}

C: Cameroonian D. praehensilis

A: D. abyssinica

P: (Western) D. praehensilis

$\mathrm{R}:$ D. rotundata 
Table S4. Likelihood comparison in fastsimcoal2

\begin{tabular}{ll}
\hline Model & $\log _{10}(L)$ \\
\hline$\{A, P\}, C\}$
\end{tabular}

$\{\{\mathrm{A}, \mathrm{P}\}, \mathrm{C}\}$

(without migration) $\quad-172110.065$

$\{\{\mathrm{C}, \mathrm{P}\}, \mathrm{A}\}$

(without migration)

$-174281.072$

$\{\{\mathrm{C}, \mathrm{A}\}, \mathrm{P}\}$

(without migration)

$-173358.592$ 


\section{Materials and Methods}

Table of contents

S1. Reference assembly

S1.1 Whole genome sequencing by Oxford Nanopore Technology

S1.2 Quality control

S1.3 De novo assembly

S1.4 Polishing and removing duplicated contigs

S1.5 Gene prediction and annotation

S2. Generation of pseudo-chromosomes by anchoring contigs onto a linkage map

S2.1 Preparing population for mapping

S2.2 Whole genome re-sequencing

S2.3 Quality control and alignment

S2.4 Identification of parental line-specific heterozygous markers

S2.5 Anchoring and ordering contigs

S3. Genetic diversity analysis

S3.1 Whole genome re-sequencing of Guinea yam accessions

S3.2 Quality control, alignment, and SNP calling

S3.3 Unsupervised clustering analysis

S3.4 Polymorphism and ploidy of nuclear genomes.

S4. Phylogenomic analysis of African yam

S4.1 Data preparation

S4.2 Neighbor-joining tree

S4.3 Inference of the evolutionary history of wild Dioscorea species by $\partial \mathrm{a} \partial \mathrm{i}$

S4.4 Inference of the evolutionary history of wild Dioscorea species by fastsimcoal2

S5. Test of hybrid origin

S5.1 Site frequency spectrum polarized by two candidate progenitors of Guinea yam

S5.2 Inference of the domestication history of Guinea yam by $\partial \mathrm{a} \partial \mathrm{i}$

S5.3 Comparison of $F_{\mathrm{ST}}$ among three African yams in each chromosome

S6. Haplotype network analysis of whole plastid genome

S7. Inference of the change of population size

S8. Exploration of extensive introgression from wild Dioscorea species 


\section{S1. Reference assembly}

\section{S1.1 Whole genome sequencing by Oxford Nanopore Technology}

To generate version 2 of Dioscorea rotundata reference genome sequence, we sequenced an F1 individual plant named "TDr96_F1" by PromethION sequencer (Oxford Nanopore Technologies). "TDr96_F1" was the same individual plant used to obtain version 1 of $D$. rotundata reference genome sequence (1). The DNA of "TDr96_F1" was extracted from fresh leaves following the proposed method (1). The DNA was subjected to size selection and purification with a gel extraction kit (Large Fragment DNA Recovery Kit; ZYMO RESEARCH). The sequencing of purified genome was performed using PromethION at GeneBay, Yokohama, Japan (http://genebay.co.jp).

\section{S1.2 Quality control}

As a first step in our pipeline for genome assembly (Fig. SM1), we removed lambda phage genome from raw reads by NanoLyse v1.1 (2). Then, we filtered out reads with average read quality score of less than 7 and those that are shorter than 1,000 bases in length by Nanofilt v2.2 (2). This was followed by trimming of the first 75 bases to remove low quality bases in all the read that were retained. This generated 3,124,439 reads, corresponding to 20.89 Gbp sequence (Table SM1).

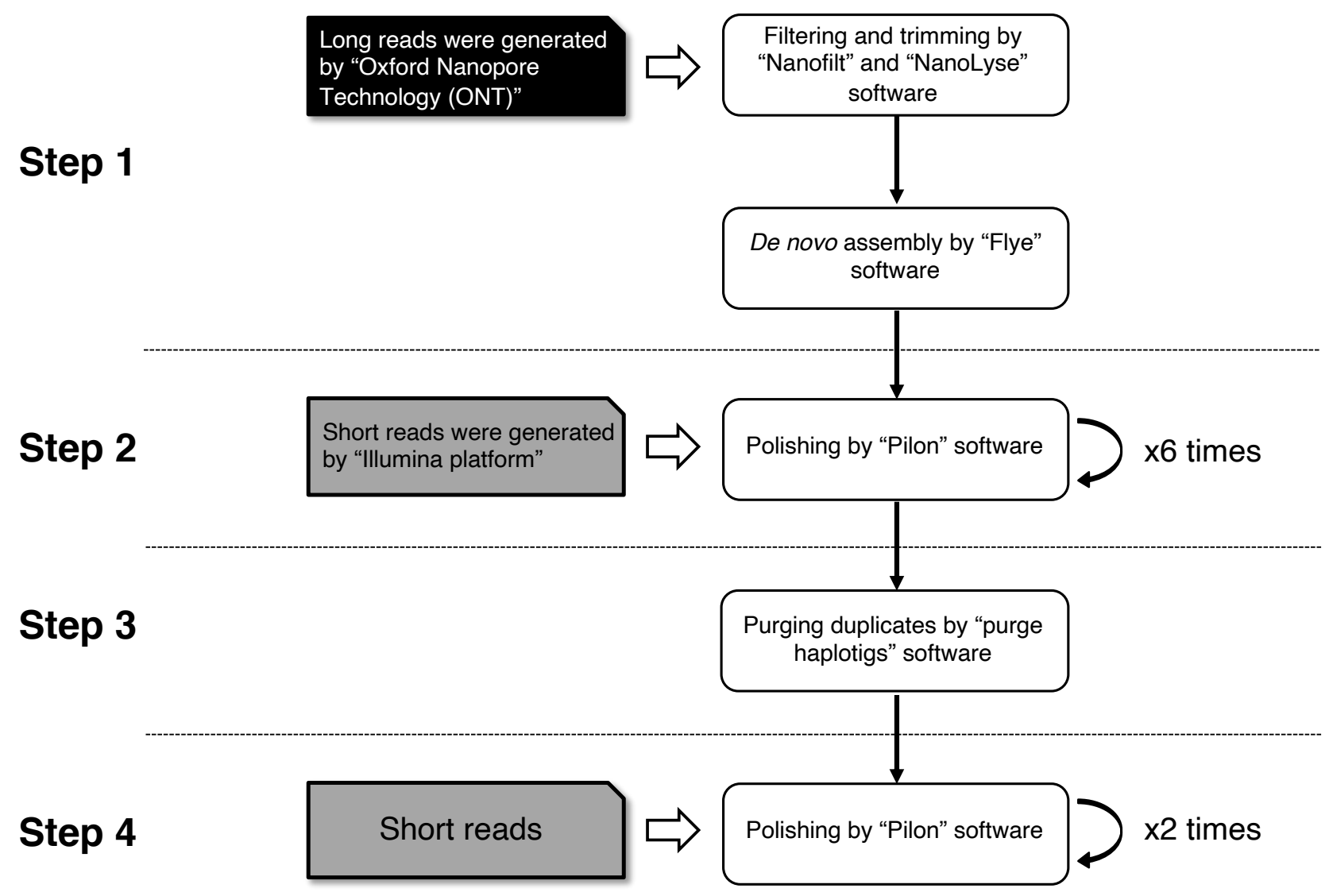

Fig. SM1. Pipeline of genome assembly Ver.2. 
Table SM1. Summary of filtered ONT reads.

\begin{tabular}{lr}
\hline Summary & \\
\hline Number of reads & $3,124,439$ \\
Total base pairs (Gbp) & 20.89 \\
Genome coverage & $36.6 \mathrm{x}$ \\
Average fragment size (Kbp) & 6.7 \\
Longest fragment & 211,597 \\
Shortest fragment & 1,000 \\
Fragment N50 (Kbp) & 8.0 \\
\hline
\end{tabular}

- Raw reads are registered in DRR196916.

- Genome coverage is estimated from the expected genome size of D. rotundata $(570 \mathrm{Mb})$.

\section{S1.3 De novo assembly}

We assembled those filtered long DNA sequence reads by Flye v2.4.2 (3), using $570 \mathrm{Mbp}$ as the estimated genome size of $D$. rotundata (1). This generated 8,721 contigs with N50 of 137,007 basepairs (Step 1 in Table SM2) and a total size of $636.8 \mathrm{Mbp}$, which is larger than the expected $D$. rotundata genome of $570 \mathrm{Mbp}$. To evaluate completeness of the gene set in the assembled contigs, we applied BUSCO analysis (Bench-Marking Universal Single Copy) v3.0.2 (4). For BUSCO analysis, we set "genome" as the assessment mode and used Embryophyta $o d b 9$ as the database and obtained 40.7\% complete BUSCOs (Step 1 in Table SM2).

Table SM2. Summary of reference assembly.

\begin{tabular}{lrrrr}
\hline & Step 1 & Step 2 & Step 3 & Step 4 \\
\hline Total number of contigs & 8,721 & 8,721 & 6,513 & $\mathbf{6 , 5 1 3}$ \\
Total base-pairs (Mbp) & 636.8 & 628.2 & 579.7 & $\mathbf{5 7 9 . 4}$ \\
Average contig size (bp) & 73,008 & 72,029 & 89,004 & $\mathbf{8 8 , 9 6 1}$ \\
Longest contig (bp) & $2,301,335$ & $2,267,833$ & $2,267,833$ & $\mathbf{2 , 2 6 7 , 3 2 6}$ \\
Shortest contig (bp) & 171 & 171 & 171 & $\mathbf{1 7 1}$ \\
N50 (bp) & 137,007 & 134,605 & 152,963 & $\mathbf{1 5 2 , 9 2 9}$ \\
& & & & \\
Complete BUSCOs (\%) & 40.7 & 89.9 & 89.3 & $\mathbf{9 0 . 1}$ \\
Complete and single-copy BUSCOs (\%) & 39.9 & 83.9 & 84.9 & $\mathbf{8 5 . 7}$ \\
Complete and duplicated BUSCOs (\%) & 0.8 & 6.0 & 4.4 & $\mathbf{4 . 4}$ \\
Fragmented BUSCOs (\%) & 8.2 & 3.2 & 3.2 & $\mathbf{3 . 1}$ \\
Missing BUSCOs (\%) & 51.1 & 6.9 & 7.5 & $\mathbf{6 . 8}$ \\
\hline
\end{tabular}

\section{S1.4 Polishing and removing duplicated contigs}

To correct the assembled contigs, we repeatedly polished them with Illumina short reads (Table SM3) using Pilon v1.23 (5) until there is no further change in \% of complete BUSCOs. We first aligned illumina jump reads as single reads to the assembled contigs by bwa mem command in BWA v0.7.17 (6) and sorted the BAM files by SAMtools v1.9 (7). The BAM files were used to run Pilon with the option "--diploid". We then polished the contigs six times. The percentage of complete BUSCOs was 
$89.9 \%$ after the first polishing step (Step 2 in Fig. SM1). To remove duplicated contigs, we used Purge Haplotigs v1.0.2 (8), which can remove duplicated contigs based on depth and number of matching bases (Step 3 in Fig. SM1). In Purge Haplotigs, the percent cutoff of aliment coverage was set to $95 \%$. After that, we polished the contigs again. Finally, the percentage of complete BUSCOs was $90.1 \%$ after the second polishing process (Step 4 in Fig. SM1). Comparing the features in old reference genome with new reference genome, the number of missing ("N") was drastically reduced (Table SM4).

Table SM3. Sequence list used in polishing.

\begin{tabular}{lcrrr}
\hline Name & Sequence Platform & $\begin{array}{r}\text { Total size } \\
(\mathbf{G b})\end{array}$ & $\begin{array}{r}\text { Genome } \\
\text { coverage }\end{array}$ & Accession No. \\
\hline $\begin{array}{l}\text { Fragment (PE) } \\
\text { MP jump reads (as Single) } \\
\text { for 2k }\end{array}$ & Illumina "Miseq" & 16.77 & $29.4 \mathrm{x}$ & DRR027644 \\
for 3k & Illumina "Hiseq 2500" & 6.43 & $11.3 \mathrm{x}$ & DRR027645 \\
for 4k & Illumina "Hiseq 2500" & 7.56 & $13.3 \mathrm{x}$ & DRR027646 \\
for 5k & Illumina "Hiseq 2500" & 6.18 & $10.8 \mathrm{x}$ & DRR027647 \\
for 6k & Illumina "Hiseq 2500" & 7.20 & $12.6 \mathrm{x}$ & DRR027648 \\
for 8k & Illumina "Hiseq 2500" & 7.27 & $12.8 \mathrm{x}$ & DRR027649 \\
\hline
\end{tabular}

- All values are calculated after quality control.

- Genome coverage is estimated from the expected genome size of D. rotundata $(570 \mathrm{Mb})$.

- In terms of jump reads, we only used the reads generated from Illumina "Hiseq 2500".

Table SM4. Comparison of old (1) and the new reference assemblies.

\begin{tabular}{lrr}
\hline Feature & Ver. $\mathbf{1}$ & Ver. 2 \\
\hline Number of scaffolds* & 4,723 & 6,513 \\
Total scaffold* size (Mbp) & 594.23 & 579.41 \\
Longest scaffold* (Mbp) & 13.61 & 2.28 \\
N50 (Mbp) & 2.12 & 0.15 \\
Total 'N' bp & $90,097,902$ & 953 \\
Complete BUSCOs (\%) & 90.7 & 90.1 \\
\hline
\end{tabular}

*In Version 2, the contigs were used instead of scaffolds.

\section{S1.5 Gene prediction and annotation}

For gene prediction, we used 20 RNA-Seq data representing 15 different organs and three different flowering stages in male and female plants (Table SM5). Total RNA was used to construct cDNA libraries using a TruSeq RNA Sample Prep Kit V2 (Illumina) according to the manufacturer's instructions. The extracted RNAs were sequenced by the Illumina platforms NextSeq500 and HiSeq4000. In the quality control step, we filtered the reads and discarded reads shorter than 50 bases and those with average read quality below 20, and trimmed poly A by FaQCs v2.08 (9). Quality trimmed reads were aligned to the newly assembled contigs by HISAT2 v2.1 (10) with options "-no-mixed --no-discordant --dta". Transcript alignments were assembled by StringTie v1.3.6 (11) for each BAM file, separately. Those GFF files were integrated by TACO v0.7.3 (12) with the option "- 
-filter-min-length 150", generating 26,609 gene models within the new assembly (Table SM6). Additionally, CDSs that were predicted using the previous reference genome (1) were aligned to the newly assembled contigs by Spaln2 v2.3.3 (13). Consequently, 8,889 CDSs that didn't have any overlap with the new gene models were added to the new gene models (Table SM6). Gene models shorter than 75 bases were removed, and InterProScan v5.36 (14) was used to predict ORFs (open reading frames) and strand information for each gene model. Finally, we predicted 35,498 genes including 66,561 transcript variants (Table SM6). For gene annotation, the predicted gene models were searched by Pfam protein family database through InterProScan (14) and by blastx command in BLAST+ (15) with option "-evalue 1e-10", using the database of Viridiplantae from UniProt as the target database. The resulting gene models and annotations were uploaded to ENSEMBL (http://plants.ensembl.org/Dioscorea rotundata/Info/Index; for early access http://stagingplants.ensembl.org/Dioscorea rotundata/Info/Index ).

Table SM5. Summary of RNA-seq data for gene prediction.

\begin{tabular}{|c|c|c|c|c|c|}
\hline \multirow[t]{2}{*}{ Sample name } & \multicolumn{2}{|l|}{ Fastq size } & \multirow[b]{2}{*}{$\begin{array}{l}\text { Sequence } \\
\text { platform }\end{array}$} & \multirow[b]{2}{*}{ Comment } & \multirow[b]{2}{*}{ Accession No. } \\
\hline & $\begin{array}{l}\text { Original } \\
\text { (Gbp) }\end{array}$ & $\begin{array}{l}\text { Filtered } \\
\text { (Gbp) }\end{array}$ & & & \\
\hline 01_Flowers-rachis-top & 4.36 & 4.28 & NextSeq500 & Top $2 \mathrm{~cm}$ of inflorescence & DRR063119 \\
\hline 02_Flowers-rachis-lower & 4.96 & 4.87 & NextSeq500 & Lower $2 \mathrm{~cm}$ of inflorescence & DRR063118 \\
\hline 03_Flower-bud & 3.52 & 3.46 & NextSeq500 & Flower bud & DRR063116 \\
\hline 04_Axillary-bud & 4.31 & 4.23 & NextSeq500 & Axillary bud & DRR063115 \\
\hline 05_Leaf & 3.26 & 3.18 & NextSeq500 & Leaf & DRR045127 \\
\hline 06_Petiole & 4.47 & 4.38 & NextSeq500 & Petiole & DRR063121 \\
\hline 07_Pulvinus & 4.66 & 4.58 & NextSeq500 & Pulvinus & DRR063120 \\
\hline 08_Rachis & 4.59 & 4.51 & NextSeq500 & Rachis & DRR063117 \\
\hline 09_Stem & 3.45 & 3.36 & NextSeq500 & Young_stem & DRR045129 \\
\hline 10_Spine & 4.51 & 4.43 & NextSeq500 & Spine & DRR063123 \\
\hline 11_Root & 3.62 & 3.54 & NextSeq500 & Root & DRR063122 \\
\hline 12_Tuber-head & 4.72 & 4.65 & NextSeq500 & Tuber (head) & DRR063126 \\
\hline 13_Tuber-middle & 4.06 & 4.00 & NextSeq500 & Tuber (middle) & DRR063125 \\
\hline 14_Tuber-tail & 4.48 & 4.40 & NextSeq500 & Tuber (tail) & DRR063124 \\
\hline 15_fem_Y917-1 & 4.12 & 4.08 & HiSeq4000 & TDr97_00917 female flower early stage 1 & DRR208398 \\
\hline 16_fem_Y917-2 & 4.27 & 4.23 & HiSeq4000 & TDr97_00917 female flower early stage 2 & DRR208399 \\
\hline 17_fem_Y917-3 & 4.43 & 4.37 & HiSeq4000 & TDr97_00917 female flower early stage 3 & DRR208400 \\
\hline 18_mal_Y777-1 & 4.48 & 4.42 & HiSeq4000 & TDr97_00777 male flower early stage 1 & DRR208401 \\
\hline 19_mal_Y777-2 & 3.43 & 3.40 & HiSeq4000 & TDr97_00777 male flower early stage 2 & DRR208402 \\
\hline 20_mal_Y777-3 & 4.13 & 4.09 & HiSeq4000 & TDr97_00777 male flower early stage 3 & DRR208403 \\
\hline
\end{tabular}


Table SM6. Summary of gene prediction.

\begin{tabular}{crr}
\hline & $\begin{array}{r}\text { Contigs } \\
\mathbf{( 6 , 5 1 3 )}\end{array}$ & $\begin{array}{r}\text { Pseudo Chrom. } \\
(\mathbf{0 1} \mathbf{2 0})\end{array}$ \\
\hline $\begin{array}{c}\text { No. genes } \\
\text { (Total transcript variants) }\end{array}$ & $\begin{array}{r}\text { (65,498 } \\
\text { ORF status }\end{array}$ & $(50,344$ \\
Complete & 22,423 & \\
5' partial & 1,225 & 19,502 \\
3' partial & 10,385 & 1,018 \\
Internal & 559 & 8,594 \\
No ORF & 906 & 465 \\
Predicted software & & 765 \\
TACO (12) & 26,609 & 23,335 \\
Spaln2 (13) & 8,889 & 7,009 \\
\hline
\end{tabular}

\section{S2. Generation of pseudo-chromosomes by anchoring contigs onto a linkage map}

\section{S2.1 Preparing population for mapping}

To develop chromosome-scale "TDr96_F1" genome sequence from the assembled contigs, we generated an F1 progeny containing $15 \overline{6}$ individuals by crossing two $D$. rotundata breeding lines: “TDr04/219", female parent (P1) and "TDr97/777", male parent (P2).

\section{S2.2 Whole genome re-sequencing}

We extracted each DNA sample from the dried leaves of $D$. rotundata following the proposed method (1). Libraries for PE short reads were constructed using an Illumina TruSeq DNA LT Sample Prep Kit (Illumina). The PE library was sequenced on the Illumina Hiseq4000 platform. Each summary of sequence and alignment is described in Table SM7 (attached at the bottom of this file).

\section{S2.3 Quality control and alignment}

We used FaQCs v2.08 (9) to remove unpaired reads and adapters. We then filtered out reads shorter than 75 bases or those whose average read quality score is 20 or lower by prinseq-lite v0.20.4 lite (16). We also trimmed bases whose average read quality score is below 20 from the 5 ' end and the 3 ' end using sliding window (the window size is five bases, and the step size is one base) in prinseqlite (16). Subsequently, we aligned the filtered reads of P1, P2, and F1 progenies to the newly assembled contigs (supplementary text $\mathrm{S} 1$ ) by bwa mem command in BWA (6). After sorting BAM files, we only retained proper paired and uniquely mapped reads by SAMtools (7).

\section{$\underline{\text { S2.4 Identification of parental line-specific heterozygous markers }}$}

\section{SNP-type heterozygous marker}

SNP-based genotypes for P1, P2, and F1 progenies were obtained as VCF file. The VCF file was generated as follows: ( i) SAMtools v1.5 (7) mpileup command with options "-t DP,AD,SP -B -Q 18 -C 50" ( ii) BCFtools v1.5 (17) call command with options "-P $0-v-m-f$ GQ,GP" (iii) BCFtools (17) view command with options "-i 'INFO/MQ $>=40 \&$ INFO/MQ0F $<=0.1 \&$ AVG(GQ) $>=10$ " (iv) 
BCFtools (17) norm command with options "-m+any" (Fig. SM2). We rejected the variants having low read depth $(<10)$ or low genotype quality score $(<10)$ in two parents. Regarding variants having low read depth $(<8)$ or low genotype quality score $(<5)$ in F1 progenies as missing, we only retained the variants having low missing rate $(<0.3)$. After that, only bi-allelic SNPs were selected by the BCFtools (17) view command with options "-m 2 -M 2 -v snps". Referring to the genotypes in the VCF file, heterozygous genotypes called by unbalanced allele frequency (out of 0.4-0.6 in two parents, and out of 0.2-0.8 in F1 progenies) were regarded as missing, and filtering for missing rate $(<0.3)$ was applied again. Finally, binomial test was applied to reject SNPs affected by segregating distortion in F1 progenies. This binomial test assumes that the probability of success rate is 0.5 on two-side hypothesis, and we regarded variants having $p$-value less than 0.2 as segregating distortion.

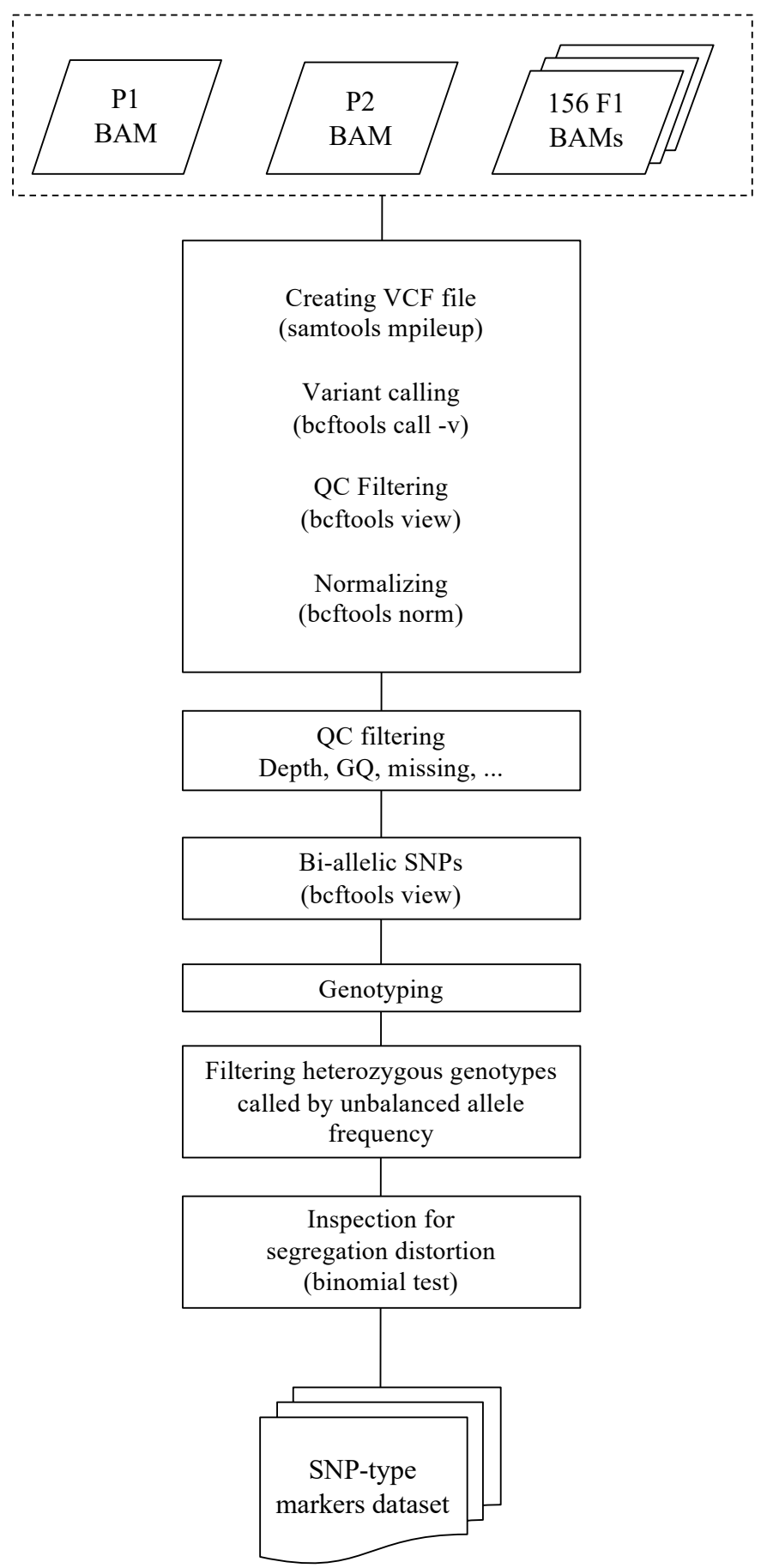

Fig. SM2. Flowchart of SNP-type heterozygous marker selection. 


\section{Presence/absence-type heterozygous marker.}

First, a VCF file was generated to search for positions having contrasting read depth between the two parental plants P1 and P2 through the following commands: (i) SAMtools (7) mpileup command with options "-B -Q 18 -C 50" ( ii) BCFtools (17) call command with option "-A" (iii) BCFtools (17) view command with options " $-\mathrm{i}$ 'MAX(FMT/DP) $>=8 \& \mathrm{MIN}(\mathrm{FMT} / \mathrm{DP})<=0$ ' -g miss -V indels". This means that one of the parents ( $\mathrm{P} 1$ or $\mathrm{P} 2)$ has enough read depth $(>=8)$ and another parent has no reads aligned on that region (A in Fig. SM3). Subsequently, we converted continuous positions in the VCF file to a feature which indicates start and end coordinate information of a region by BEDTools v.2.26 (18) merge command with options "-d 10 -c 1 -o count". After that, we only retained sufficiently wide feature ( $>=50 \mathrm{bp}$ ) in the BED file (the 1st BED). To reject false-positives whereby low depth regions are erroneously regarded as absence regions, we focused on both the boundary regions around each feature and features themselves. For boundary regions, the 2nd BED file including expanded (twice-sized) features of each feature given in the 1st BED was generated by BEDTools (18) slop command with options "-b 0.5 -pct". Using depth value in each feature given in the 1st BED, presence/absence-based genotypes for parental plants P1, P2, and F1 progenies were determined. For verification to reject the false-positive features, we also referred to the depth values in the boundary regions around each feature. Verified features were only accepted as presence/absence markers. The depth values in each feature were calculated by SAMtools (7) bedcov command with option "-Q 0". Also, the depth values in the boundary regions were obtained by subtracting the depth values of the 2nd BED from that of the 1st BED (B in Fig. SM3). For P1 and P2, we regarded genotypes having depth $>=8$ as presence genotype meaning the heterozygosity of presence and absence, while those having depth $<2$ were classified as absence genotypes meaning the homozygosity of absence. For F1 progenies, we classified markers having depth $>0$ and $=0$ as presence and absence markers, respectively. Finally, we applied the same binomial test in SNP-type heterozygous markers as in the presence/absence-type heterozygous markers.

A

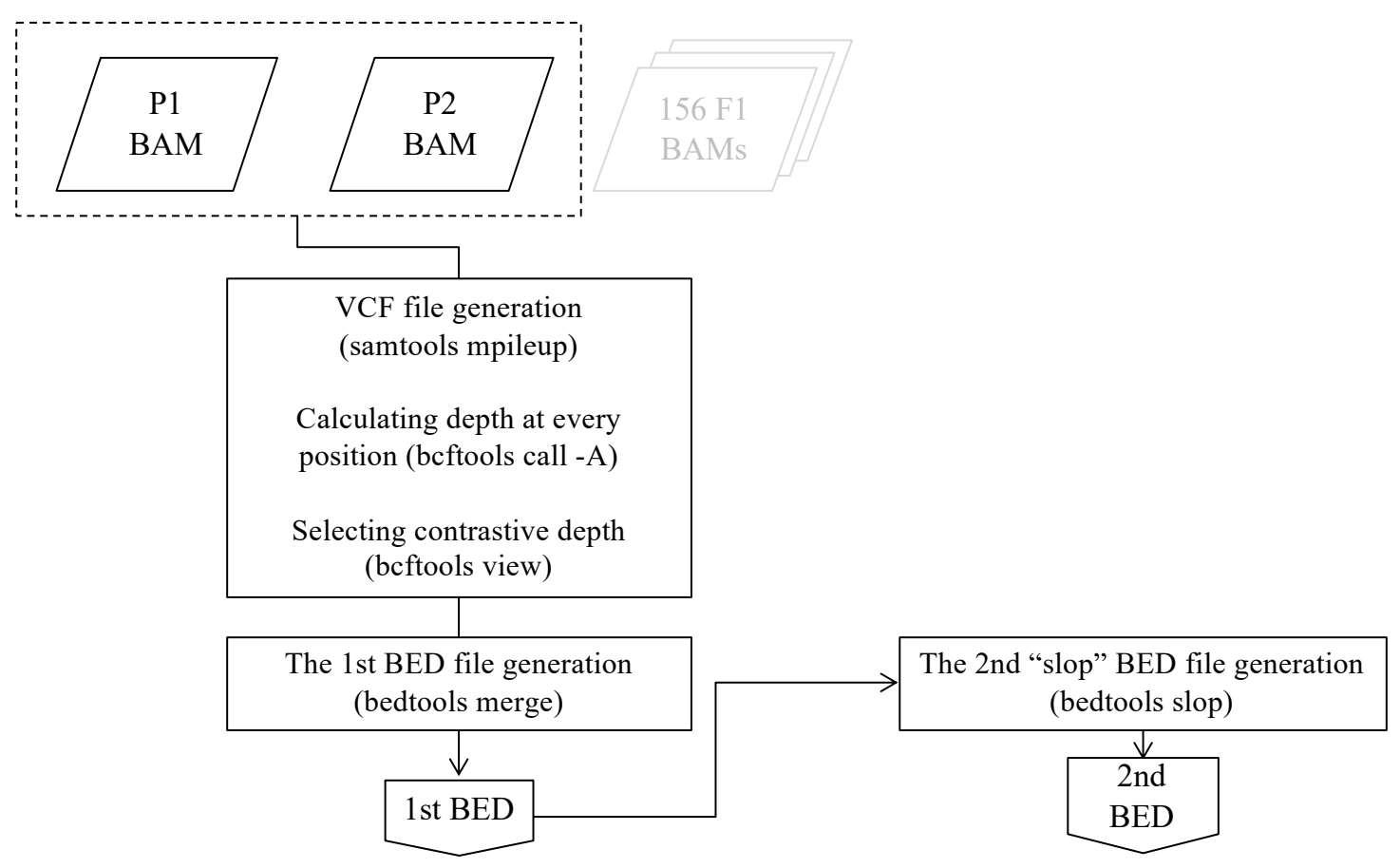

(continued) 


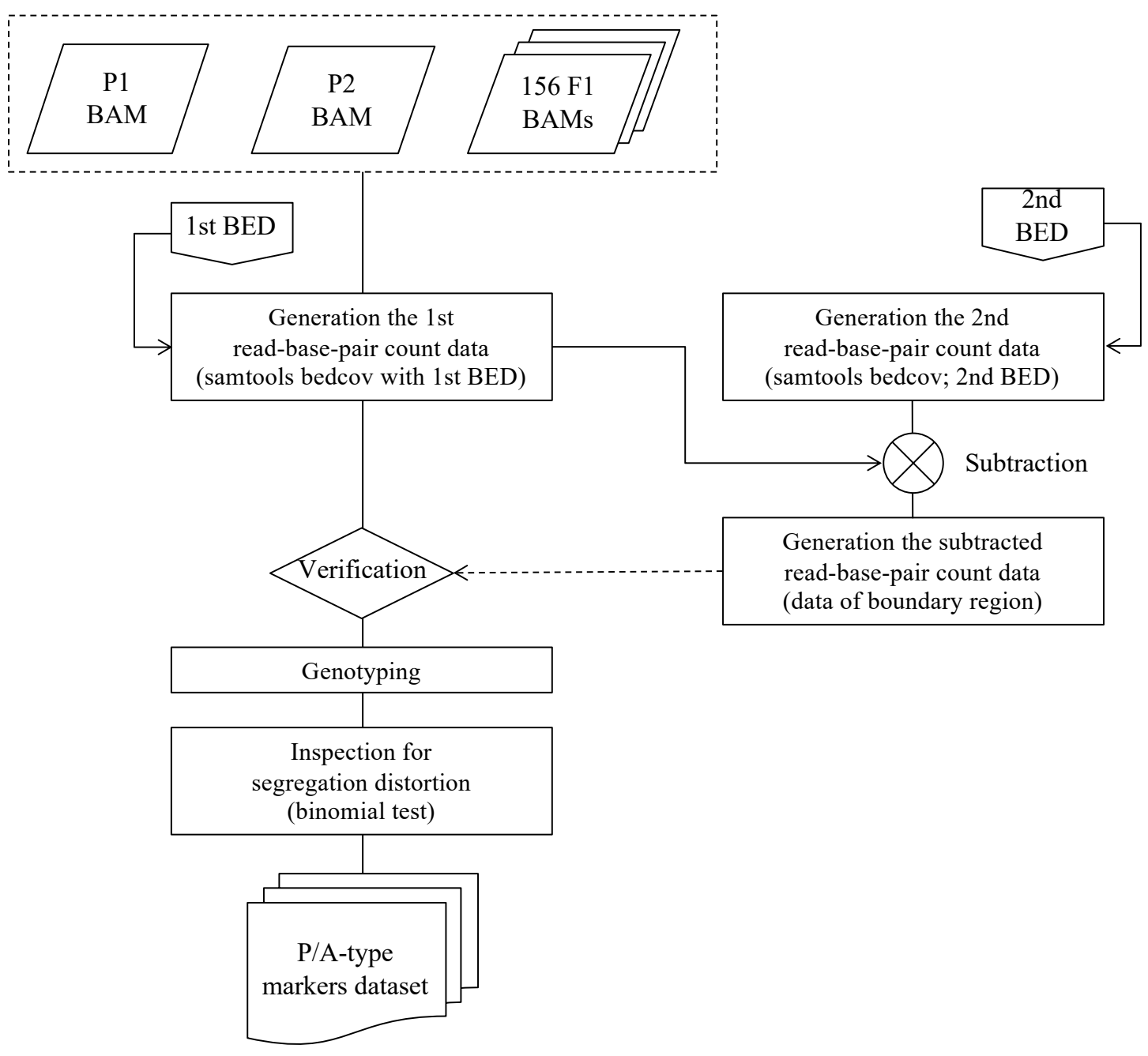

Fig. SM3. Flowchart of presence/absence-type heterozygous marker selection.

Integration of SNP-type and presence/absence-type heterozygous markers. To develop parental line-specific linkage maps, we integrated SNP-type and P/A-type (presence/absence-type) heterozygous makers. Two types of markers, Type- 1 markers and Type- 2 makers, were defined. If a SNP-type marker is heterozygous in P1 but homozygous in $\mathrm{P} 2$ or if a P/A-type maker is present in $\mathrm{P} 1$ and absent in P2, it is classified as Type-1 marker (P1-heterozygous marker set). Conversely, if a SNP-type marker is homozygous and heterozygous in P1 and P2, respectively or if a P/A-type maker is absent in $\mathrm{P} 1$ but present in $\mathrm{P} 2$, it is classified as Type-2 marker (P2-heterozygous marker set).

\section{$\underline{\text { S2.5 Anchoring and ordering contigs }}$}

Pruning and flanking markers by Spearman's correlation coefficients. Distance matrices of Spearman's correlation coefficients $(\rho)$ were calculated for every marker pair in each contig in each marker set (P1-heterozygous marker set and P2-heterozygous marker set). According to the histogram of absolute $\rho$ calculated from each contig, most markers on the same contigs were correlated with each other (Fig. SM4). Therefore, we pruned correlated flanking markers to remove redundant markers (Fig. SM5). Accordingly, we obtained 11,389 markers for linkage mapping (Table SM8). 


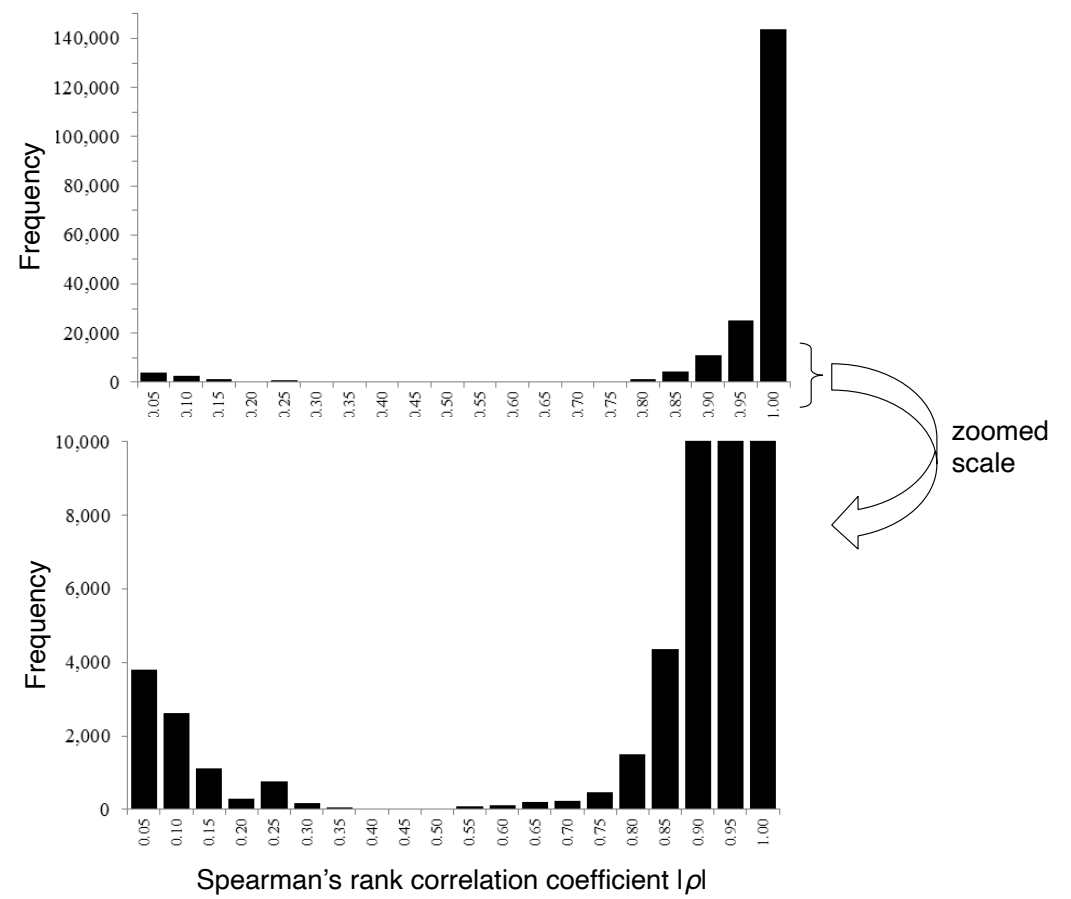

Fig. SM4. Histogram of absolute values of $\rho$ calculated from each contig.

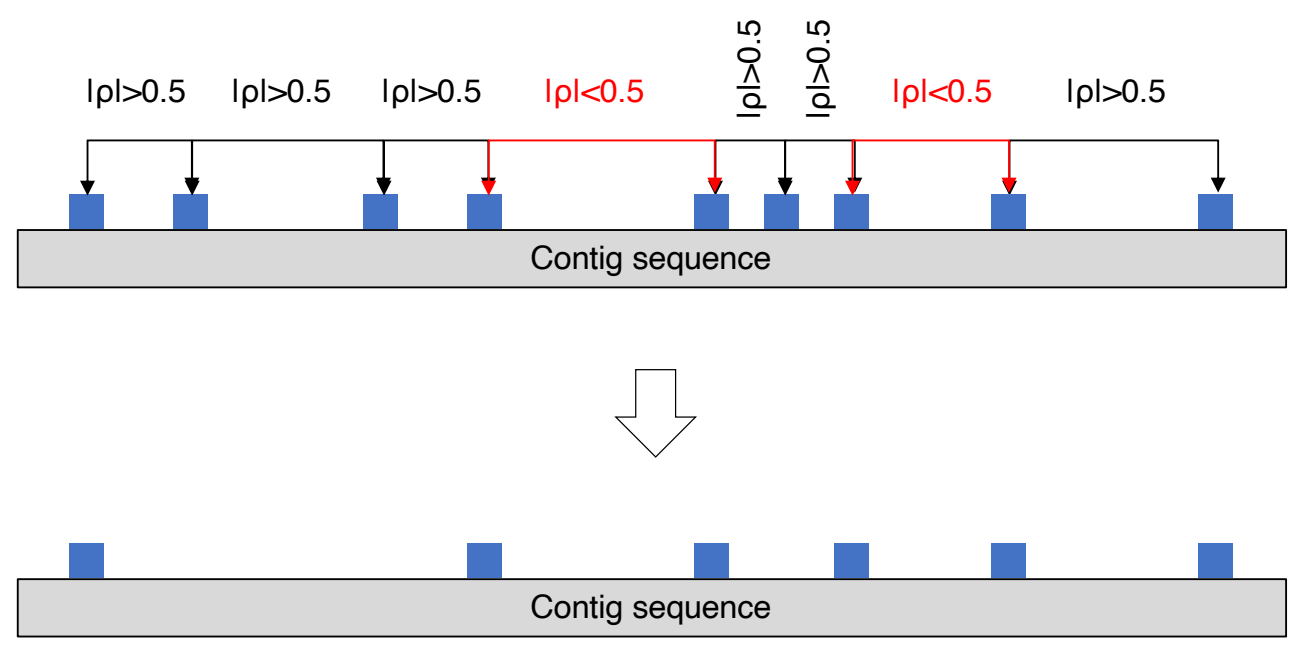

Fig. SM5. A process of pruning correlated flanking markers.

Table SM8. Summary of the anchoring makers.

\begin{tabular}{lrrr}
\hline & Type1 & Type2 & Type1 + Type2 \\
\hline Total anchoring markers to make linkage group & 7,020 & 4,369 & 11,389 \\
- SNP & 4,607 & 3,435 & 8,042 \\
- P/A & 2,413 & 934 & 3,347 \\
& & & \\
Total base pairs of linkage group having markers (Mbp) & 434.7 & 328.4 & 495.2 \\
Total anchored base pairs estimated from genome size (\%) & 75.5 & 56.7 & 85.5 \\
\hline
\end{tabular}


Linkage mapping. The markers obtained from the previous section were converted to the genotypeformatted data. Based on that genotype-formatted data, genetic linkage maps were constructed by MSTmap (19) with following parameter set: "population_type DH; distance_function kosambi; cut_off_p_value 0.000000000001 ; no_map_dist 15.0 ; no_map_size 0 ; missing threshold 25.0; estimation_before_clustering no; detect_bad_data no; objective_function ML" for each marker set. After trimming the orphan linkage groups, we solved the complemented-phased duplex linkage groups caused by coupling-type and repulsion-type markers in pseudo-testcross method. Finally, two parental-specific linkage maps were constructed. These two linkage maps were designated as P1-map (which was constructed using Type-1 marker) and P2-map (which was constructed using Type-2 marker) (Fig SM6 and Fig SM7). The linkage groups were visualized by r/qtl (20). The numbering of linkage groups is the same as the previous reference genome (1).

A

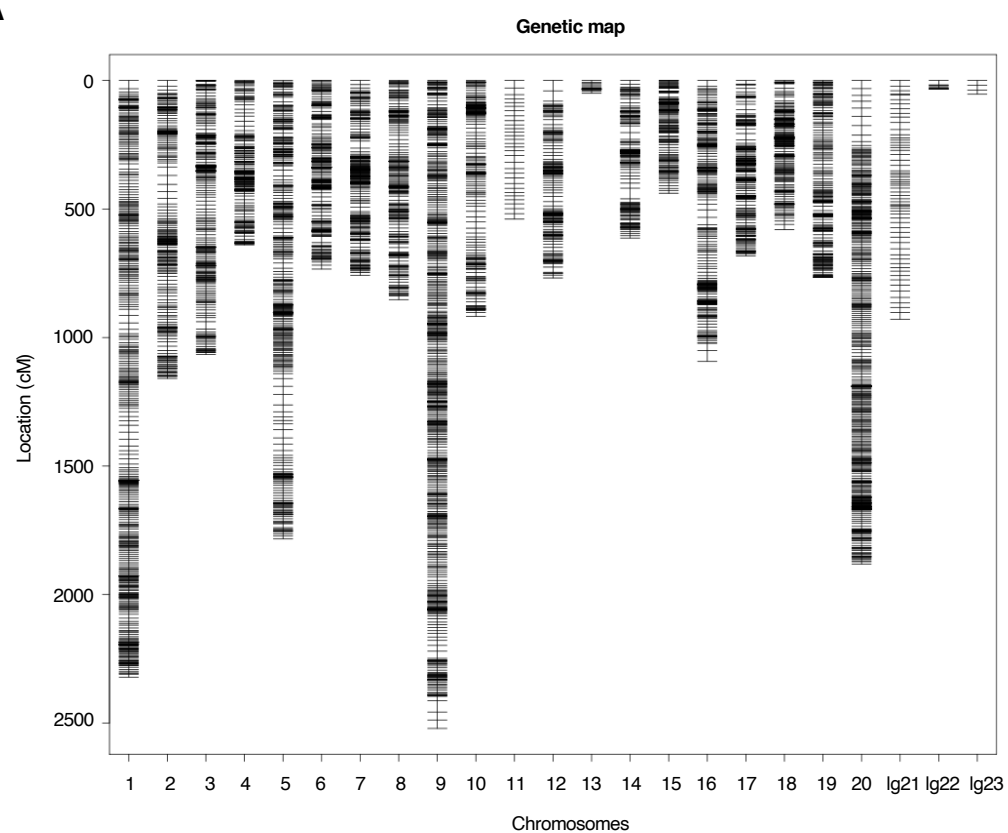

B

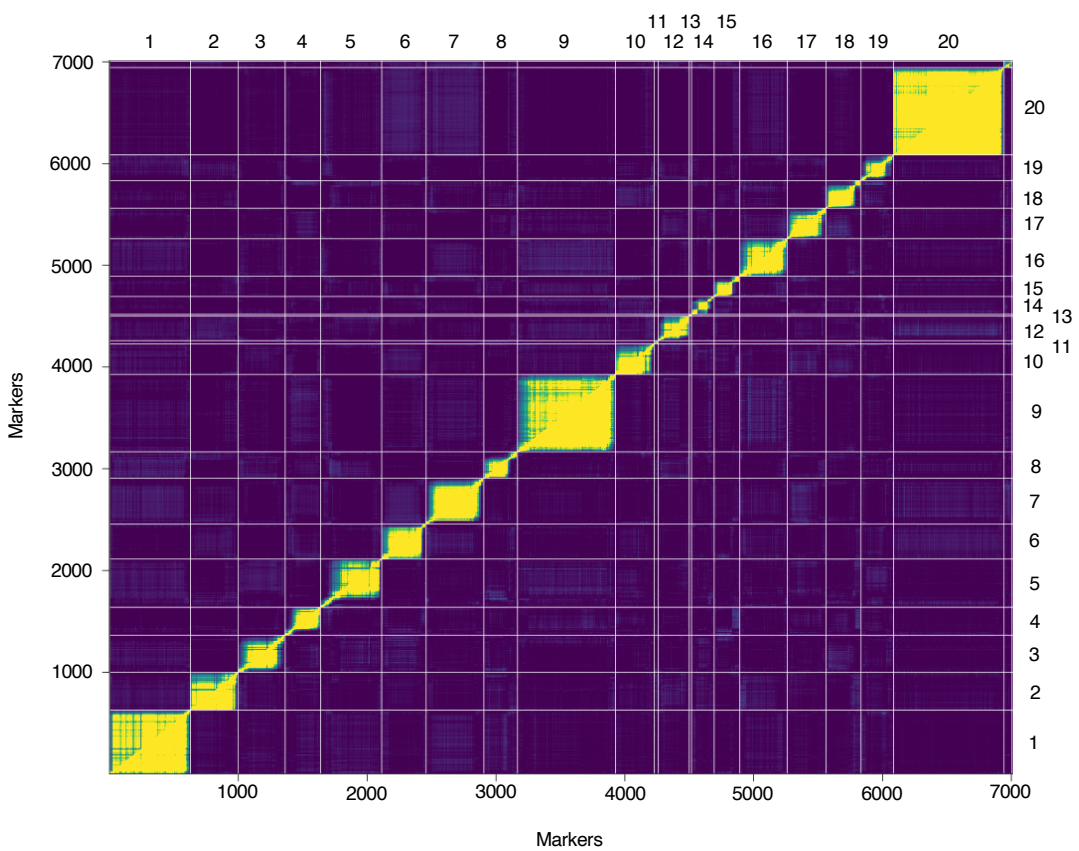

Fig. SM6. P1-map created by P1 heterozygous markers. (A) Contig positions in P1-map. (B) Estimated recombination fractions (upper-left triangle) against LOD score (low-right triangle) plotted by R/qtl (20). 


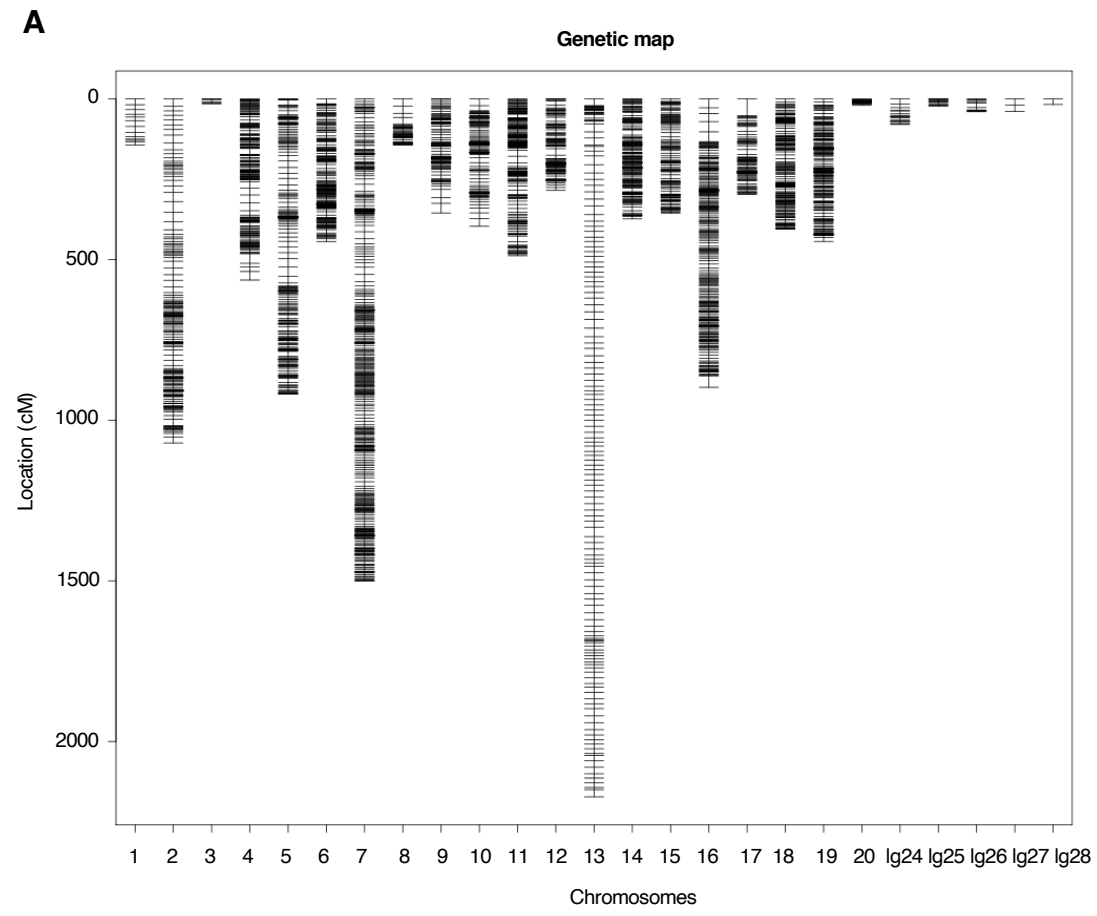

B

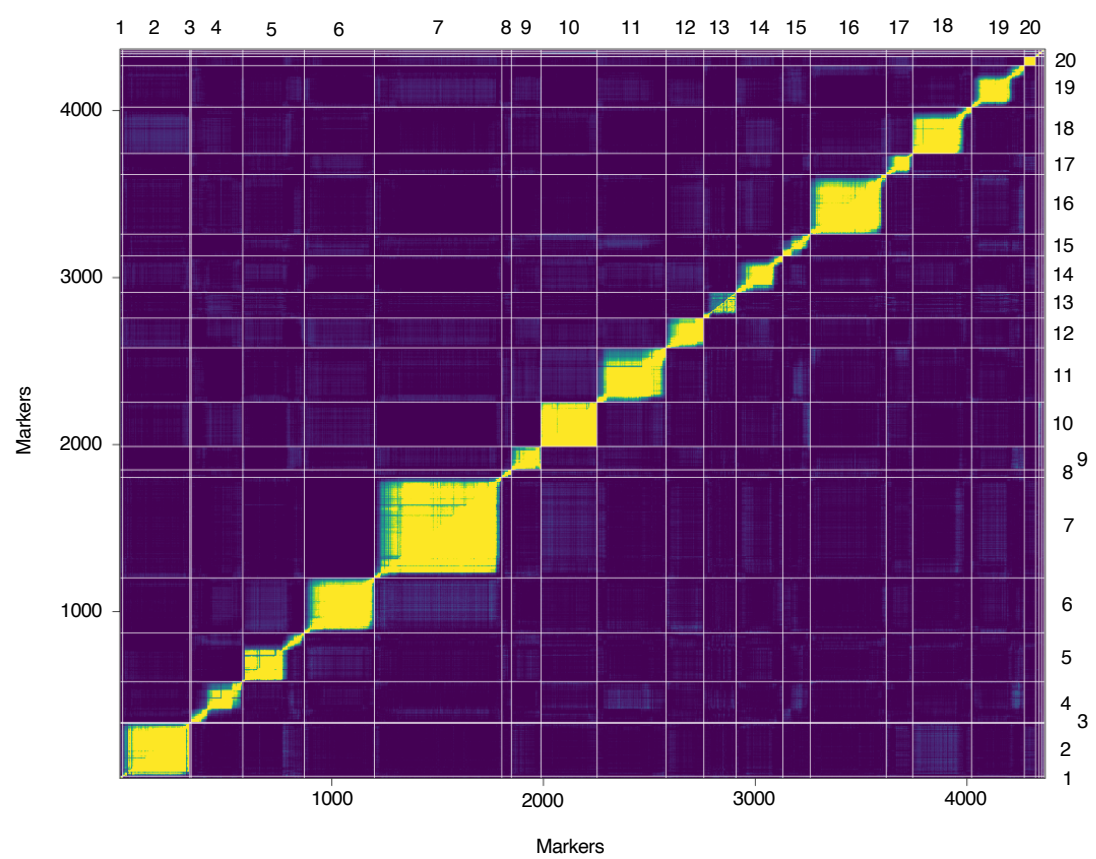

Fig. SM7. P2-map created by $P 2$ heterozygous markers. (A) Contig positions in P2-map. (B) Estimated recombination fractions (upper-left triangle) against LOD score (low-right triangle) plotted by R/qtl (20).

Integration of two parental-specific linkage maps to chromosome-scaled physical genome sequence. Based on a matrix derived from those contigs that are shared between P1- and P2-map, linkage groups (Table SM9), the contigs were anchored and linearly ordered as pseudo-chromosomes. During the anchoring and ordering process, we identified contigs whose markers were allocated to different linkage groups. Such contigs were further divided into sub-contigs to ensure that they were 
not allocated to different pseudo-chromosomes. To divide the contigs at the proper position, we followed a previously proposed method (1). During this procedure, 34 genes including 61 transcript variants were cut and removed. The previously proposed method (1) was followed to finally generate the pseudo physical genome sequence composed of 20 pseudo-chromosomes. To compare the newly generated pseudo-chromosomes with the one we constructed previously (1), we generated a dot plot by D-Genies (21) (Fig. SM8) and counted the anchored base pairs in the new pseudo-chromosomes (Table SM10). The resulting reference genome including unanchored contigs was uploaded to ENSEMBL (http://plants.ensembl.org/Dioscorea rotundata/Info/Index; for early access http://staging-plants.ensembl.org/Dioscorea rotundata/Info/Index ).

Table SM9. A matrix of the number of the shared contigs between P1-map and P2-map. Linkage group (lg) 21-28 don't have the shared contigs.

\begin{tabular}{|c|c|c|c|c|c|c|c|c|c|c|c|c|c|c|c|c|c|c|c|c|c|c|c|c|c|c|c|}
\hline & & P2-ma & & & & & & & & & & & & & & & & & & & & & & & & & \\
\hline & & 1 & 2 & 3 & 4 & 5 & 6 & 7 & 8 & 9 & 10 & 11 & 12 & 13 & 14 & 15 & 16 & 17 & 18 & 19 & 20 & $\lg 24$ & $\lg 25$ & $\lg 26$ & $\lg 27$ & $\lg 28$ & \\
\hline P1-map & 1 & & 2 & & 1 & 0 & 3 & 2 & 0 & 0 & 3 & 2 & 1 & 0 & 1 & 0 & 5 & 0 & 2 & 0 & 1 & 0 & 0 & $\begin{array}{ll}0 & 0\end{array}$ & $0 \quad 0$ & $0 \quad$ & \\
\hline & 2 & & 120 & & 0 & 2 & 2 & 3 & 0 & 1 & 1 & 1 & 0 & 0 & 0 & 0 & 1 & 0 & 1 & 2 & 0 & 0 & 0 & 0 & 0 & 0 & \\
\hline & 3 & 0 & 2 & & 3 & 0 & 3 & 9 & 0 & 1 & 0 & 0 & 0 & 0 & 0 & 0 & 1 & 0 & 1 & 2 & 0 & 0 & 0 & 0 & 2 & & \\
\hline & 4 & 0 & 0 & & 84 & 2 & 0 & 1 & 0 & 0 & 0 & 0 & 0 & 0 & 3 & 0 & 1 & 0 & 0 & 0 & 0 & 0 & 0 & 0 & 0 & 0 & \\
\hline & 5 & 0 & 1 & 0 & & $\begin{array}{ll}3 & 135 \\
\end{array}$ & 2 & 3 & 0 & 1 & 1 & 2 & 2 & 0 & 4 & 1 & 0 & 1 & 1 & 2 & 0 & 0 & 0 & 0 & 0 & 0 & \\
\hline & 6 & 0 & 0 & 0 & & $\begin{array}{ll}0 & 3\end{array}$ & 128 & 2 & 0 & 1 & 1 & 2 & 0 & 0 & 1 & 0 & 2 & 0 & 0 & 2 & 0 & 0 & 0 & 0 & 0 & & \\
\hline & 7 & 0 & 2 & 0 & & 2 & 2 & 199 & 0 & 1 & 1 & 3 & 0 & 0 & 0 & 1 & 1 & 0 & 0 & 3 & 0 & 0 & 0 & 0 & 0 & 0 & \\
\hline & 8 & 0 & 0 & 0 & 1 & 1 & 4 & 1 & 24 & 0 & 0 & 0 & 0 & 0 & 0 & 1 & 4 & 1 & 2 & 1 & 0 & 9 & 0 & 0 & 0 & 0 & \\
\hline & 9 & 0 & 1 & 0 & & 2 & 4 & 4 & 0 & 71 & 4 & 1 & 0 & 0 & 2 & 1 & 5 & 1 & 0 & 1 & 0 & 0 & 0 & 0 & 0 & 0 & \\
\hline & 10 & 0 & 1 & 0 & & 0 & 0 & 1 & 0 & 0 & 93 & 1 & 1 & 0 & 1 & 1 & 0 & 1 & 0 & 0 & 0 & 0 & 6 & 0 & 0 & 0 & \\
\hline & 11 & 0 & 0 & 0 & & 0 & 0 & 1 & 0 & 0 & 0 & 8 & 0 & 0 & 0 & 0 & 0 & 0 & 1 & 0 & 0 & 0 & 0 & 0 & 0 & & \\
\hline & 12 & 0 & 0 & 0 & & 2 & 0 & 1 & 0 & 0 & 2 & 2 & 75 & 1 & 0 & 1 & 2 & 0 & 5 & 0 & 0 & 0 & 0 & 0 & 0 & & \\
\hline & 13 & 0 & 0 & 0 & & 0 & 0 & 1 & 0 & 0 & 0 & 0 & 0 & 5 & 0 & 0 & 0 & 0 & 0 & 0 & 0 & 0 & 0 & 0 & 0 & & \\
\hline & 14 & 0 & 0 & 0 & & 1 & 1 & 1 & 0 & 0 & 2 & 0 & 0 & 1 & 66 & 0 & 0 & 1 & 0 & 1 & 1 & 0 & 0 & 0 & 0 & 0 & 0 \\
\hline & 15 & 0 & 0 & 0 & & 0 & 0 & 0 & 0 & 1 & 0 & 1 & 0 & 0 & 2 & 42 & 2 & 0 & 0 & 1 & 0 & 0 & 0 & 0 & 0 & 0 & 0 \\
\hline & 16 & 0 & 1 & 0 & & 2 & 0 & 2 & 0 & 2 & 0 & 1 & 1 & 0 & 0 & 0 & 126 & 1 & 1 & 0 & 0 & 0 & 0 & 0 & 0 & & \\
\hline & 17 & 0 & 0 & 0 & & 2 & 1 & 1 & 0 & 0 & 0 & 1 & 0 & 1 & 1 & 1 & 2 & 60 & 0 & 0 & 0 & 0 & 0 & 0 & 0 & 0 & 0 \\
\hline & 18 & 0 & 1 & 0 & & 0 & 2 & 1 & 0 & 0 & 1 & 2 & 1 & 0 & 0 & 0 & 0 & 0 & 118 & 0 & 0 & 0 & 0 & 0 & 0 & & 0 \\
\hline & 19 & 0 & 1 & 0 & 0 & 0 & 1 & 2 & 0 & 0 & 0 & 2 & 0 & 4 & 0 & 0 & 0 & 0 & 1 & 100 & 0 & 0 & 0 & 0 & 0 & & 0 \\
\hline & 20 & 1 & 8 & 0 & & 5 & 1 & 4 & 0 & 0 & 5 & 6 & 2 & 3 & 2 & 0 & 4 & 1 & 1 & 0 & 39 & 0 & 0 & 3 & 0 & & 0 \\
\hline & $\lg 21$ & 0 & 0 & 0 & 0 & 0 & 0 & 1 & 0 & 0 & 0 & 0 & 0 & 1 & 0 & 0 & 0 & 0 & 0 & 0 & 0 & 0 & 0 & 0 & 0 & 0 & 0 \\
\hline & $\lg 22$ & 0 & 0 & 0 & & 0 & 0 & 0 & 0 & 0 & 0 & 6 & 0 & 0 & 0 & 0 & 0 & 0 & 0 & 0 & 0 & 0 & 0 & 0 & 0 & 0 & 0 \\
\hline & $\lg 23$ & 0 & 0 & 0 & 0 & 0 & 0 & 0 & 0 & 0 & 0 & 1 & 0 & 0 & 0 & 0 & 0 & 0 & 0 & 0 & 0 & 0 & 0 & 0 & 0 & & \\
\hline
\end{tabular}

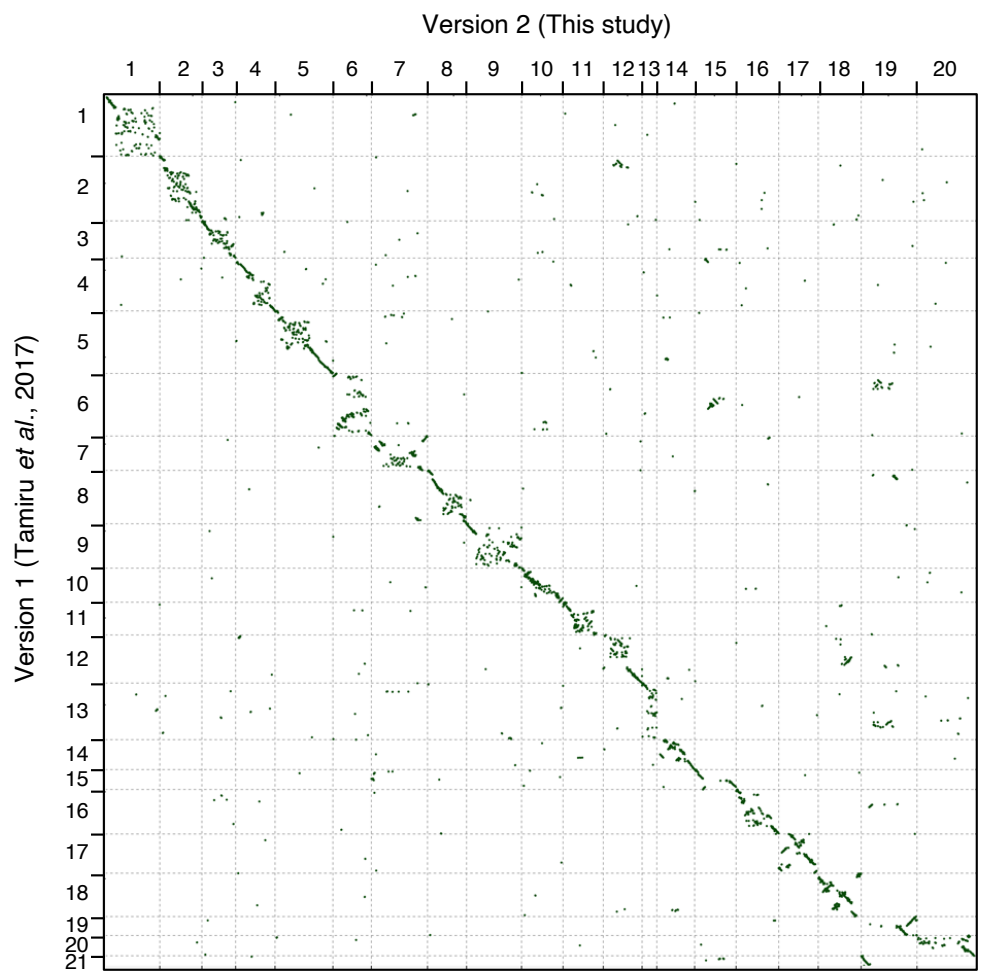

Fig. SM8. Dot plot of the new pseudo-chromosomes (Ver.2) against the previous pseudochromosomes (Ver.1) (1). 
Table SM10. Comparison of old (Ver. 1) (1) and new (Ver. 2) pseudo-chromosomes.

\begin{tabular}{lrr}
\hline Feature & Ver. 1 & Ver. 2 \\
\hline Number of Pseudo Chr. & 21 & 20 \\
Total size of Pseudo Chr. (Mbp) & 456.67 & 491.97 \\
Total not 'N' Mbp & 406.1 & 487.31 \\
Total size of Pseudo Chr. / Total scaffold* (\%) & 76.9 & 84.9 \\
Complete BUSCOs (\%) & $82.8 \%$ & $82.3 \%$ \\
\hline
\end{tabular}

*In version2, the contigs were used instead of scaffolds.

\section{S3. Genetic diversity analysis}

\section{S3.1 Whole genome re-sequencing of Guinea yam accessions}

For genetic diversity analysis, we selected 333 accessions of D. rotundata maintained at IITA, Nigeria, representing the genetic diversity of Guinea yam landraces and improved lines of West Africa. We extracted DNA from the dried leaves of each accession of D. rotundata following the proposed method (1). Libraries for PE short reads were constructed using an Illumina TruSeq DNA LT Sample Prep Kit (Illumina). The PE library was sequenced on the Illumina Nextseq500 or Hiseq4000 platform. Finally, P1 (TDr04/219) and P2 (TDr97/777) parents used to anchor the contigs and the reference individual "TDr96_F1" were added to 333 accessions. Therefore, we totally used 336 accessions for this analysis. Summary of sequences and alignments are given in Table S1.

\section{S3.2 Quality control, alignment, and SNP calling}

We used FaQCs v2.08 (9) and prinseq-lite v0.20.4 lite (16) for quality control. We used the same parameters provided in supplementary text S2.3, but both of paired and unpaired reads were aligned to the new reference genome by bwa mem command in BWA (6) with option "-a". After sorting the BAM files, the VCF file was generated by SAMtools (7) mpileup command with options "-t DP,AD,SP -B -Q 18 -C 50", and variants were called by BCFtools (17) call command with options “-P 0 -v -m -f GQ,GP”. Low quality variants were rejected by BCFtools (17) view command with options "-i 'INFO/MQ $>=40 \&$ INFO/MQ0F $<=0.1 \& \mathrm{AVG}(\mathrm{GQ})>=5$ "'. Regarding variants having low read depth $(<8)$ or low genotype quality score $(<5)$ as missing, we filtered the SNPs having high missing rate $(>=0.3)$ across all samples and only retained bi-allelic SNPs on the pseudochromosomes.

\section{S3.3 Unsupervised clustering analysis}

$6,124,093$ SNPs were retained in 336 Guinea yam accessions through the pipeline of supplementary text S3.2. The VCF file including 336 Guinea yam accessions was converted into GDS file by gdsfmt v1.20 R package implemented in SNPRelate v1.18 (22) R package. After that, we ran SNPRelate (22), without filtering, for PCA (principal component analysis). Moreover, we used sNMF v1.2 (23) for admixture analysis of the 336 Guinea yam accessions. To choose the best value of $K$, we launched sNMF (23) for each value of $K$ from 2 to 20 (Fig. SM9). We couldn't find the best value of $K$ based on cross-entropy criterion, but we defined five cluster for convenience. 


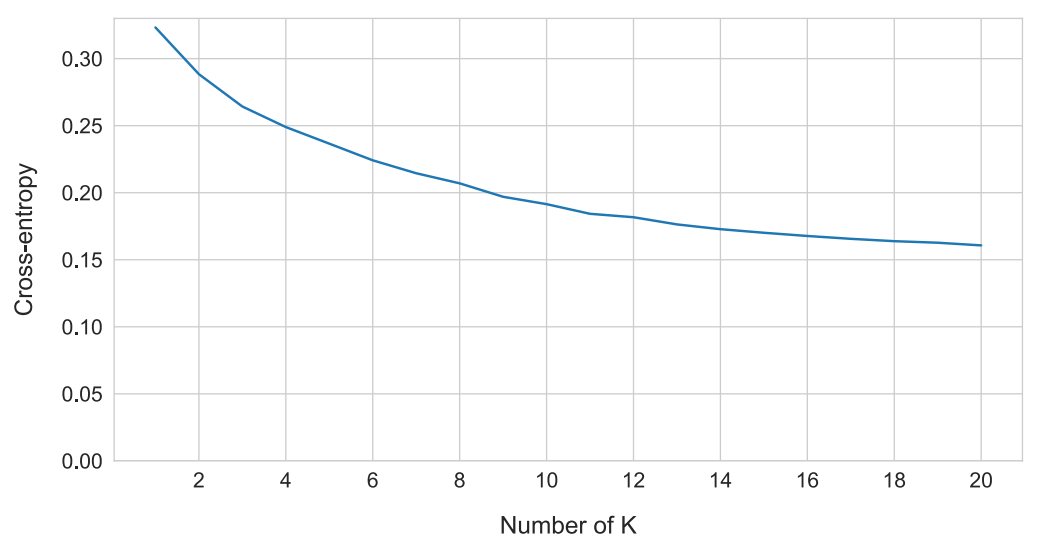

Fig. SM9. Cross-entropy values from $K=1$ to $K=20$ for admixture analysis.

\section{$\underline{\text { S3.4 Polymorphism and ploidy of nuclear genomes }}$}

Heterozygosity ratio and unique alleles. First, we calculated the heterozygosity ratio in each accession (Fig. S1). We defined the heterozygosity ratio as follows:

$$
(\text { Heterozygosity ratio })=\frac{S}{L}
$$

where $S$ is the number of heterozygous SNPs and $L$ is the number of mapped sites in an accession. Second, we counted the unique alleles in each cluster (Fig. S2). An allele is considered unique if it only exits in a cluster even when the allele is a singleton in all accessions.

\section{Flow cytometry.}

Ploidy level was estimated by flow cytometry using a Partec Ploidy Analyzer (Sysmex Partec, Gorlitz, Germany). Fully developed fresh young leaves were sampled and chopped (ca. $5 \mathrm{~mm}$ x 5mm) using a razor blade with $0.4 \mathrm{~mL}$ nuclear extraction buffer (solution A of a high-resolution kit; Sysmex Partec, Gorlitz, Germany). The suspension was filtered through a nylon filter (50- $\mu \mathrm{m}$ mesh), and the extracted nuclei were stained with 4',6-diamino-2-phenylindole solution. After let stand for 5 min at room temperature, the sample was applied for ploidy analyzer at a rate of 5-20 nuclei/s. The DNA index (DI) of each accession was calculated based on the relative amount of DNA in nuclei at the G1 stage compared with that of internal standard. Rice (Oryza sativa L.) was used as an internal standard for calibration of the measurements. Flow cytometry was repeated two or three times with different leaf samples to confirm the DI of each accession. The ploidy levels of each accession were determined by comparing their DI with that of the diploid accession, "TDr1673", for which the chromosome number was confirmed microscopically as $2 n=40$. (Table S1)

Summary statistics in population genetics. After removing the accessions of cluster 1 due to triploid accessions, we imputed missing genotypes by BEAGLE v4.1 (24) with default options. After that, summary statistics in population genetics were calculated (Table S2). Firstly, we counted segregating sites and singletons in 308 Guinea yam accessions. We also estimated Watterson's $\theta\left(\hat{\theta}_{W}\right)(25)$, pairwise nucleotide diversity $\left(\hat{\theta}_{\pi}\right)(26)$, and Tajima's $D(27)$ in the same dataset. We defined $\hat{\theta}_{W}$ as follows:

$$
\hat{\theta}_{W}=\frac{S}{a * \bar{L}}
$$


where $a$ is equal to:

$$
a=\sum_{i=1}^{n-1} \frac{1}{i}
$$

and $\bar{L}$ is number of average mapped sites in a population and $n$ is a number of sequences.

Also, we defined that $\hat{\theta}_{\pi}$ is equal to:

$$
\hat{\theta}_{\pi}=\frac{1}{\bar{L}} \frac{n}{n-1} \frac{\sum_{i<j} k_{i j}}{n(n-1) / 2}
$$

where $\bar{L}$ is the number of average mapped sites in a population, $n$ is the number of sequences, and $k_{i j}$ is number nucleotide differences between the $i$ th and $j$ th sequences.

\section{S4. Phylogenomic analysis of African yam}

\section{S4.1 Data preparation}

For phylogenomic analysis of African yam, we used 308 Guinea yam accessions sequenced in the present study excluding cluster 1 triploid accessions, as well as 80 D. rotundata, 29 D. abyssinica, 21 Western D. praehensilis, and 18 Cameroonian D. praehensilis as sequenced in the previous study (28) using two accessions of Asian species D. alata as an outgroup (Table SM11). In terms of the samples sequenced in the previous study (28), we only used the sequences whose species labels matched a species predicted by the admixture analysis in the previous study (28). Also, we removed the sequences which were labeled as hybrid in the previous study (28). Two sequences of D. alata for outgroup were downloaded from NCBI (Table SM11). Subsequently, read quality control, alignment, and SNP calling of those 458 sequences were conducted through the same pipeline in supplementary text S3.2. Except for the Neighbor-joining (NJ) tree (29) (supplementary text S4.2), we only used the SNPs which have the missing rate less than 0.3 in each targeted species. When the markers are polarized by $D$. alata, the SNPs at the positions where the alleles of $D$. alata were not completely fixed or where either of the sequences of $D$. alata was missing were filtered out.

\section{S4.2 Neighbor-joining tree}

Before constructing NJ tree (29), we only retained SNPs at positions having no missing data across all five species (D. rotundata, D. abyssinica, Western D. praehensilis, Cameroonian D. praehensilis, and D. alata). When we converted the VCF file including the rest SNPs to multi-FASTA file, heterozygous SNPs were converted to IUPAC code to characterize them as ambiguous markers. To construct the NJ tree (29), we ran MEGA X v10.1.8 (30) using the rest 463,293 SNPs. In MEGA X (30), the bootstrap value was set to 100 and the other parameters were set as default. Finally, the resulting file was drawn by GGTREE v2.0.4 (31).

\section{S4.3 Inference of the evolutionary history of wild Dioscorea species by $\partial \mathrm{a} \partial \mathrm{i}$}

To elucidate the evolutionary relationships of the three wild Dioscorea species, D. abyssinica (indicated as A), Western D. praehensilis (P). Cameroonian D. praehensilis (C) that are closely related to $D$. rotundata, we adopted $\partial \mathrm{a} \partial \mathrm{i}$ analysis (32), which allows estimating evolutionary parameters from an unfolded site frequency spectrum. The joint unfolded site frequency spectrum was calculated from the 17,532 polarized SNPs, and it was projected down to 25 chromosomes in each species.

First, three phylogenetic models, $\{\{\mathrm{A}, \mathrm{P}\}, \mathrm{C}\},\{\{\mathrm{C}, \mathrm{P}\}, \mathrm{A}\},\{\{\mathrm{C}, \mathrm{A}\}, \mathrm{P}\}$ were tested without considering migration among the species. The parameter bounds of each population size was ranged from $10^{-3}$ to 100 , and those of each divergence time was ranged from 0 to 3 , which were suggested 
in the manual of $\partial \mathrm{a} \partial \mathrm{i}$ (https://dadi.readthedocs.io/en/latest/). The grid size was set to $(40,50,60)$. The maximum iteration for an inference was set to 20 . Randomly perturbing the initial values by 'perturb_params' function in $\partial \mathrm{a} \partial \mathrm{i}$ (32), the parameters were inferred 100 times. On these conditions, the $\{\{\mathrm{A}, \mathrm{P}\}, \mathrm{C}\}$ had the highest likelihood out of the three models (Table. S3).

Based on the assumption that $\{\{\mathrm{A}, \mathrm{P}\}, \mathrm{C}\}$ is the true evolutionary relationship among the three wild Dioscorea species, the evolutionary parameters were re-estimated by $\partial \mathrm{a} \partial \mathrm{i}$ (32) allowing symmetric migration among the species. Then, the parameter bounds of each symmetric migration rate were ranged from 0 to 20 , which was also suggested in the manual of $\partial \mathrm{a} \partial \mathrm{i}$. The parameters were inferred 100 times by $\partial \mathrm{a} \partial \mathrm{i}(32)$ with the different initial parameters, and the best parameter set was selected based on Akaike information criterion.

\section{S4.4 Inference of the evolutionary history of wild Dioscorea species by fastsimcoal2}

To complement our result and to exactly replicate the previous report (28), fastsimcoal2 (33) used in the previous study $(28)$ was also used to test these three models $(\{\{\mathrm{A}, \mathrm{P}\}, \mathrm{C}\},\{\{\mathrm{C}, \mathrm{P}\}, \mathrm{A}\}$, and $\{\{\mathrm{C}$, A $\}, P\})$. Until the step of SNP calling, we basically followed our own pipeline in supplementary text S3.2 based on the reference genome version 1 including the unanchored contigs (1) to be consistent with the previous study (28). The misclassified samples excluding hybrids were genetically reclassified by the admixture analysis following the previous study (28). The threshold of missing rate across all samples was set to 0.25 which was proposed in the previous study (28). The resulting SNPs through our pipeline were 87,672, which were less than the number of the analyzed SNPs in the coalescent simulation of the previous study (28). Therefore, we skipped the down sampling of the SNPs to 100,000 unlike the previous study (28). In other steps and the parameter bounds for the coalescent simulation by fastsimcoal2 (33), we exactly followed the method proposed in the previous study (28) using the same version of fastsimcoal2 (33).

\section{S5. Test of hybrid origin}

\section{S5.1 Site frequency spectrum polarized by two candidate progenitors of Guinea yam}

We focused on the allele frequencies of 388 D. rotundata sequences including 80 in the previous study (28) at the SNPs positioned over the entire genome and are oppositely fixed in the two candidate progenitors. The SNP set was generated by following supplementary text S4.1. Based on this SNP set, 144 SNPs were oppositely fixed in the two candidate progenitors across all pseudo-chromosomes, and allele frequencies of these 144 SNPs were calculated and plotted.

\section{S5.2 Inference of the domestication history of Guinea yam by $\partial \mathrm{a} \partial \mathrm{i}$}

To infer the domestication history of Guinea yam, $\partial \mathrm{a} \partial \mathrm{i}(32)$ was adopted. Using the 15,461 polarized SNPs generated by following supplementary text $\mathrm{S} 4.1$, three phylogenetic models, $\{\{\mathrm{A}, \mathrm{R}\}, \mathrm{P}\},\{\{\mathrm{P}$, $\mathrm{R}\}, \mathrm{A}\},\{\{\mathrm{A}, \mathrm{R}\},\{\mathrm{P}, \mathrm{R}\}\}$ (hypothesis 1,2 , and 3 in Fig. $2 \mathrm{~A}$, respectively) were tested with considering symmetric migration among the species. The parameter bound for the admixed proportion from $D$. abyssinica was ranged from 0 to 1 . The other parameter bounds were same to supplementary text S4.3. The maximum iteration for an inference was set to 20 . The parameters were inferred 100 times by $\partial \mathrm{a} \partial \mathrm{i}(32)$.

\section{$\underline{\text { S5.3 Comparison of } \boldsymbol{F}_{S T}}$ among three African yams in each chromosome}

$F_{S T}(34)$ among the three species (D. abyssinica, (Western) D. praehensilis, and D. rotundata) was calculated in each chromosome. We estimated the $F_{S T}$ from:

$$
F_{S T}=\frac{H_{T}-H_{S}}{H_{T}}
$$

where $H_{T}$ and $H_{S}$ are the expected heterozygosity in total population and sub-divided population, respectively, and are equal to: 


$$
\begin{gathered}
H_{T}=2 \frac{f_{A 1}+f_{A 2}}{2}\left(1-\frac{f_{A 1}+f_{A 2}}{2}\right) \\
H_{S}=\frac{2 f_{A 1}\left(1-f_{A 1}\right)+2 f_{A 2}\left(1-f_{A 2}\right)}{2}=f_{A 1}\left(1-f_{A 1}\right)+f_{A 2}\left(1-f_{A 2}\right)
\end{gathered}
$$

where $f_{A 1}$ and $f_{A 2}$ are the allele frequencies in each population (34). Finally, the calculated $F_{S T}$ were averaged in each chromosome.

\section{S6. Haplotype network analysis of whole plastid genome}

The sample set used to construct the haplotype network of the whole plastid genome was same to that in NJ tree (supplementary text S4.2). We aligned the 458 whole genome sequences, together with the whole plastid genome of $D$. rotundata $(1)$, to the newly improved reference genome of $D$. rotundata. We basically followed the pipeline described in supplementary text S3.2 for quality control and alignment. Because plastid genome is haploid, "--ploidy" option was set to 1 in BCFtools call command (17) when SNPs were called. Singleton SNPs were removed as unreliable markers. Also, SNPs having more than one low quality genotype $(\mathrm{GQ}<127)$ across the samples were also removed as unreliable markers. We didn't allow any missing. Finally, haplotype network was constructed by median joining network algorithm (35) implemented in PopART (36).

\section{S7. Inference of the change of population size}

To understand the change of population sizes, demographic history of African yams was re-inferred by $\partial \mathrm{a} \partial \mathrm{i}$ (32) allowing migration. By fixing the parameters predicted in supplementary text S5.2 except for the population sizes, we re-estimated each population size at the start and end points after the emergence of those species assuming an exponential increase/decrease of the population sizes. The parameter bounds of population sizes were ranged from $10^{-3}$ to 100 , and the maximum iteration for an inference was set to 20 . The parameters were inferred 100 times by $\partial \mathrm{a} \partial \mathrm{i}(32)$.

\section{S8. Exploration of extensive introgression from Dioscorea species}

To explore the possibility of multiple introgression from both parental wild yams, $f_{4}$ statistic $(37,38)$ was applied to the four clusters of $D$. rotundata excluding cluster 1 triploid accessions. Here, $f_{4}$ statistic needs four populations; $\mathrm{P}_{\mathrm{R} 1}$ is the first cluster of $D$. rotundata; $\mathrm{P}_{\mathrm{R} 2}$ is the second cluster of $D$. rotundata; $\mathrm{P}_{\mathrm{P}}$ is a population of (Western) D. praehensilis; $\mathrm{P}_{\mathrm{A}}$ is a population of D. abyssinica. We estimated $\hat{f}_{4}\left(P_{R 1}, P_{R 2}, P_{P}, P_{A}\right)$ from the following formula using a sliding window analysis with window size of $250 \mathrm{Kbp}$ and step size of $25 \mathrm{Kbp}$ :

$$
\hat{f}_{4}\left(P_{R 1}, P_{R 2}, P_{P}, P_{A}\right)=\left(\hat{p}_{R 1}-\hat{p}_{R 2}\right)\left(\hat{p}_{P}-\hat{p}_{A}\right)
$$

where $\hat{p}_{j}$ is the observed allele frequency in a window in population $\mathrm{P}_{j}$.

In most windows, $\hat{f}_{4}$ is close to zero, which means that the window has a concordant genealogy because the two clusters of $D$. rotundata have a small genetic distance (B in Fig. SM10). However, if these two clusters of $D$. rotundata have a large genetic distance and if one of or both populations have a small genetic distance from a wild Dioscorea species, then $\hat{f}_{4}$ skews from 0 . Therefore, a locus having a skewed $\hat{f}_{4}$ has a discordant genealogy (C or D in Fig. SM10). For $\mathrm{P}_{\mathrm{P}}$ (the population of $D$. praehensilis) and $\mathrm{P}_{\mathrm{A}}$ (the population of $D$. abyssinica), the samples sequenced in the previous study (28) were used (Table SM11), and the dataset was prepared by following supplementary text S4.1. As the first screening, all possible combinations of the clusters of D. rotundata, excluding accessions in cluster 1 , were used for $\mathrm{P}_{\mathrm{R} 1}$ and $\mathrm{P}_{\mathrm{R} 2}$ (Fig. S11). In this analysis, we identified an extensive introgression around the SWEETIE gene (4.00Mbp $\sim 4.15 \mathrm{Mbp}$ on chromosome 17$)$. Because cluster 2 and 5 have the same genealogy pattern around the SWEETIE gene, we merged them into one population $\left(\mathrm{P}_{25}\right)$ and we used this as $\mathrm{P}_{\mathrm{R} 1}$. Because cluster 4 has the opposite genealogy pattern 
to $\mathrm{P}_{25}$ around the SWEETIE gene, we used $\mathrm{P}_{4}$ as $\mathrm{P}_{\mathrm{R} 2}$. As a result, $\hat{f}_{4}\left(P_{25}, P_{4}, P_{P}, P_{A}\right)$ was calculated for the second screening (Fig. 4). If a locus has $\left|Z\left(f_{4}\right)\right|>5$, we regarded it as an outlier (red dots in Fig. 4B). To reveal relationship of the D. rotundata accessions around the SWEETIE gene, Neighbor-Net was constructed by SplitsTree v5.1.4 (39) using 308 D. rotundata accessions excluding the accessions in cluster 1, 29 D. abyssinica, and 21 D. praehensilis. A total 458 variants from $4.00 \mathrm{Mbp}$ to $4.15 \mathrm{Mbp}$ region on chromosome 17 were converted to multi-FASTA. At that time, heterozygous genotypes were converted to IUPAC code.

A Equation for $f_{4}$

$$
2 f_{4}\left(P_{R_{1}}, P_{R_{2}}, P_{P}, P_{A}\right)=\mathbb{E} T_{R_{1} P}+\mathbb{E} T_{R_{2} A} \quad-\quad \mathbb{E} T_{R_{1} A} \quad \text { } \quad \mathbb{E} T_{R_{2} P}
$$

B Concordant genealogy of $f_{4}$
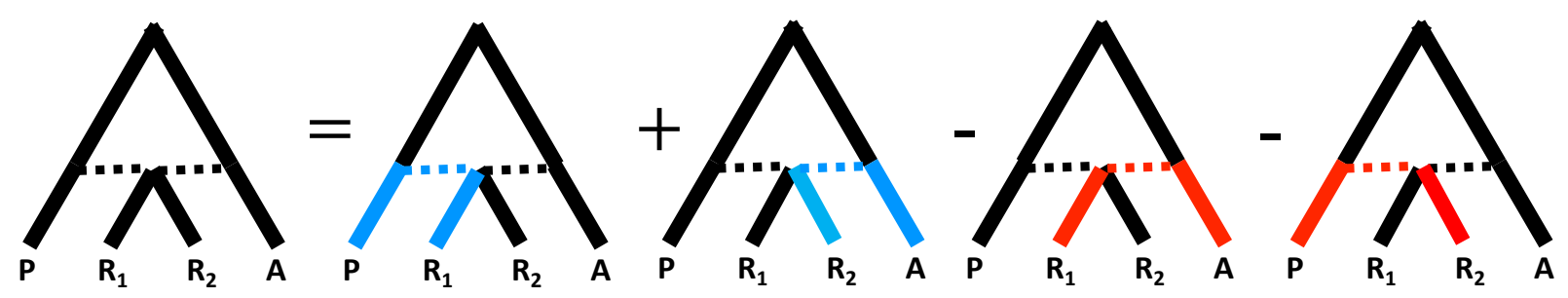

C Discordant genealogy of $f_{4}$ (ABBA)

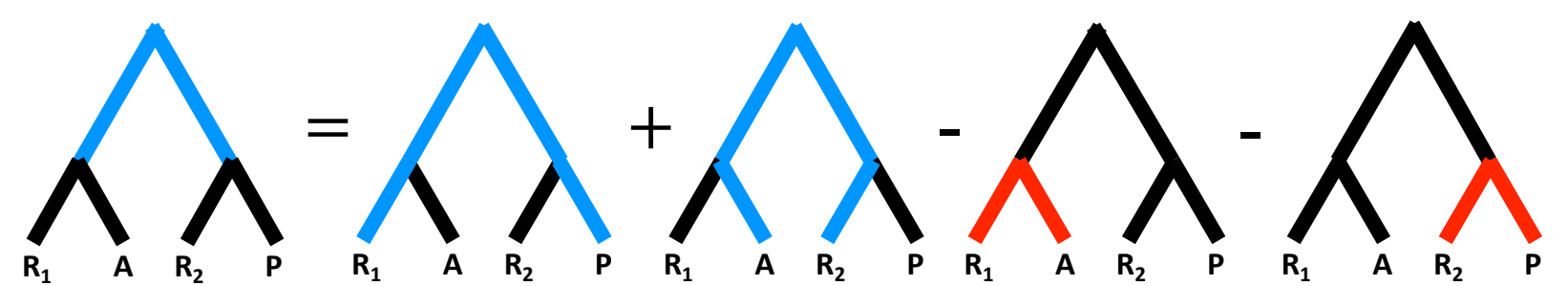

D Discordant genealogy of $f_{4}(\mathrm{BABA})$

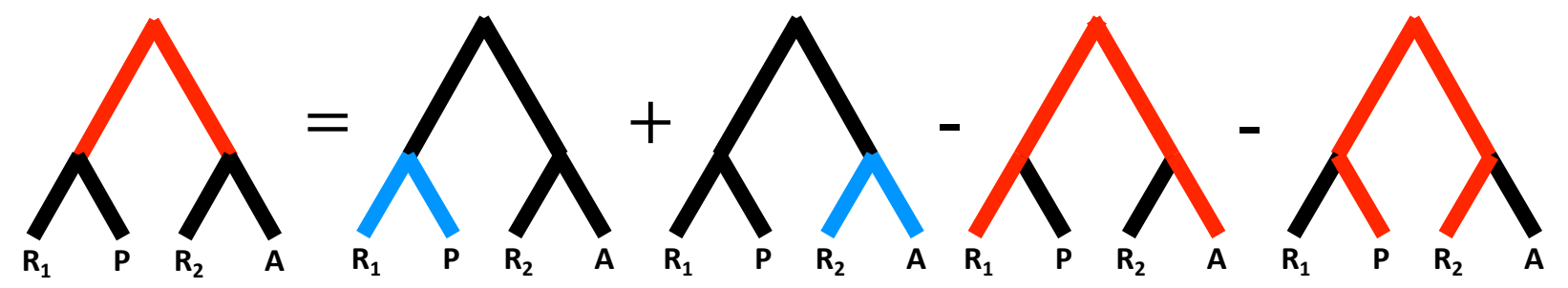

Fig. SM10. Schematic explanation how $f_{4}$ behaves in this study adapted from (38). "A" represents the population of $D$. abyssinica. "P" represents the population of $D$. praehensilis. "R1" represents the first populations of $D$. rotundata. "R2" represents the second populations of $D$. rotundata. 

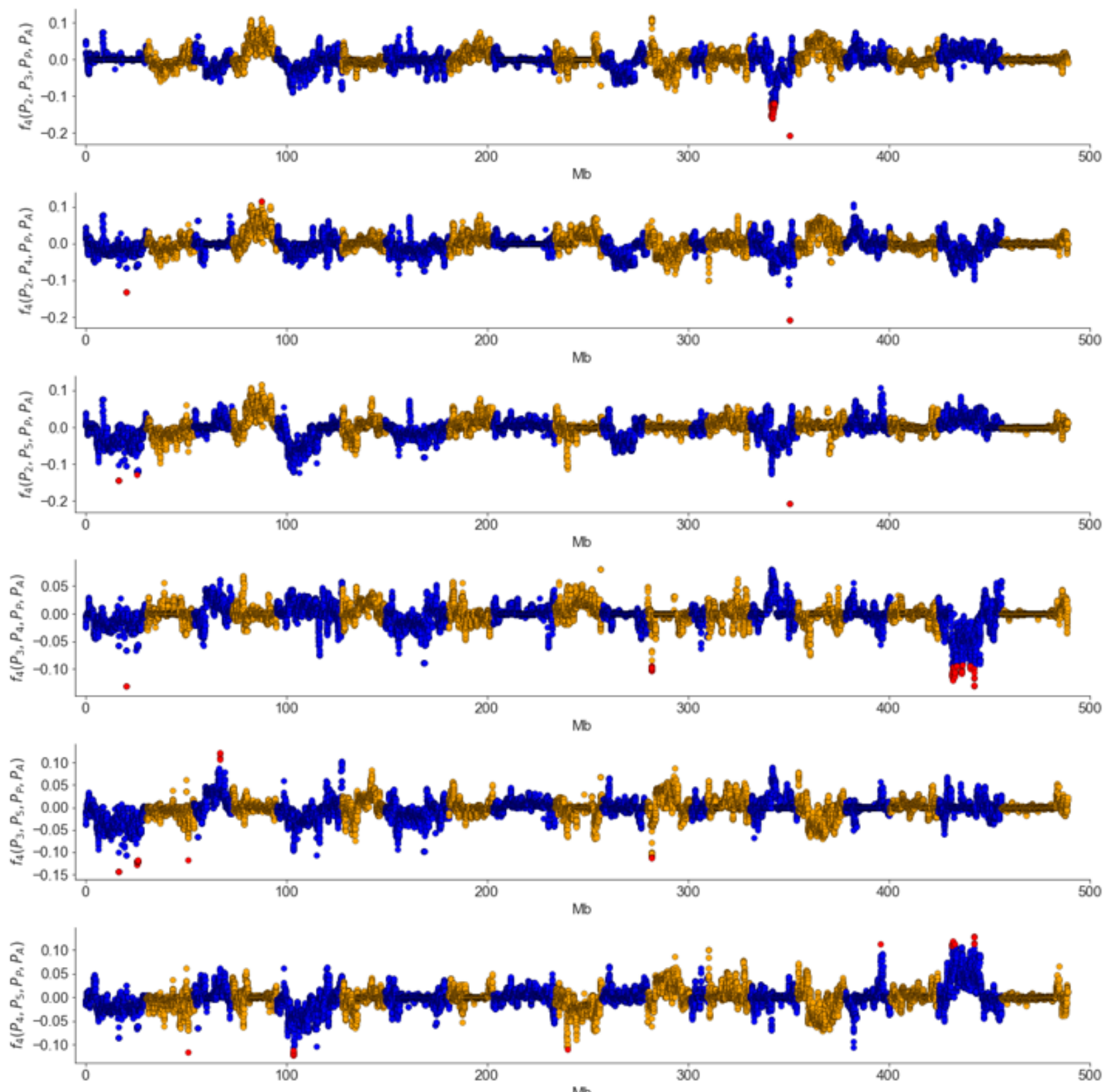

Fig. S11. $f_{4}$ in all possible combinations of clusters excluding cluster1. Population $\mathrm{P} i$ means a population of the cluster $i$.

1. M. Tamiru, S. Natsume, H. Takagi, B. White, H. Yaegashi, M. Shimizu, K. Yoshida, A. Uemura, K. Oikawa, A. Abe, N. Urasaki, H. Matsumura, P. Babil, S. Yamanaka, R. Matsumoto, S. Muranaka, G. Girma, A. Lopez-Montes, M. Gedil, R. Bhattacharjee, M. Abberton, P. L. Kumar, I. Rabbi, M. Tsujimura, T. Terachi, W. Haerty, M. Corpas, S. Kamoun, G. Kahl, H. Takagi, R. Asiedu, R. Terauchi, Genome sequencing of the staple food crop white Guinea yam enables the development of a molecular marker for sex determination. BMC Biol. 15, 86 (2017).

2. W. De Coster, S. D’Hert, D. T. Schultz, M. Cruts, C. Van Broeckhoven, NanoPack: visualizing and processing long-read sequencing data. Bioinformatics. 34, 2666-2669 (2018).

3. M. Kolmogorov, J. Yuan, Y. Lin, P. A. Pevzner, Assembly of long, error-prone reads using repeat graphs. Nat. Biotechnol. 37, 540-546 (2019). 
4. F. A. Simão, R. M. Waterhouse, P. Ioannidis, E. V. Kriventseva, E. M. Zdobnov, BUSCO: assessing genome assembly and annotation completeness with single-copy orthologs. Bioinformatics. 31, 3210-3212 (2015).

5. B. J. Walker, T. Abeel, T. Shea, M. Priest, A. Abouelliel, S. Sakthikumar, C. A. Cuomo, Q. Zeng, J. Wortman, S. K. Young, A. M. Earl, Pilon: An Integrated Tool for Comprehensive Microbial Variant Detection and Genome Assembly Improvement. PLoS ONE. 9, e112963 (2014).

6. H. Li, R. Durbin, Fast and accurate short read alignment with Burrows-Wheeler transform. Bioinformatics. 25, 1754-1760 (2009).

7. H. Li, B. Handsaker, A. Wysoker, T. Fennell, J. Ruan, N. Homer, G. Marth, G. Abecasis, R. Durbin, The Sequence Alignment/Map format and SAMtools. Bioinformatics. 25, 2078-2079 (2009).

8. M. J. Roach, S. A. Schmidt, A. R. Borneman, Purge Haplotigs: allelic contig reassignment for third-gen diploid genome assemblies. BMC Bioinform. 19, 460 (2018).

9. C.-C. Lo, P. S. G. Chain, Rapid evaluation and quality control of next generation sequencing data with FaQCs. BMC Bioinform. 15, 366 (2014).

10. D. Kim, B. Langmead, S. L. Salzberg, HISAT: a fast spliced aligner with low memory requirements. Nat. Methods. 12, 357-360 (2015).

11. M. Pertea, G. M. Pertea, C. M. Antonescu, T.-C. Chang, J. T. Mendell, S. L. Salzberg, StringTie enables improved reconstruction of a transcriptome from RNA-seq reads. Nat. Biotechnol. 33, 290-295 (2015).

12. Y. S. Niknafs, B. Pandian, H. K. Iyer, A. M. Chinnaiyan, M. K. Iyer, TACO produces robust multisample transcriptome assemblies from RNA-seq. Nat. Methods. 14, 68-70 (2017).

13. H. Iwata, O. Gotoh, Benchmarking spliced alignment programs including Spaln2, an extended version of Spaln that incorporates additional species-specific features. Nucleic Acids Res. 40, e161 (2012).

14. P. Jones, D. Binns, H.-Y. Chang, M. Fraser, W. Li, C. McAnulla, H. McWilliam, J. Maslen, A. Mitchell, G. Nuka, S. Pesseat, A. F. Quinn, A. Sangrador-Vegas, M. Scheremetjew, S.-Y. Yong, R. Lopez, S. Hunter, InterProScan 5: genome-scale protein function classification. Bioinformatics. 30, 1236-1240 (2014).

15. C. Camacho, G. Coulouris, V. Avagyan, N. Ma, J. Papadopoulos, K. Bealer, T. L. Madden, BLAST+: architecture and applications. BMC Bioinformatics. 10, 421 (2009).

16. R. Schmieder, R. Edwards, Quality control and preprocessing of metagenomic datasets. Bioinformatics. 27, 863-864 (2011).

17. H. Li, A statistical framework for SNP calling, mutation discovery, association mapping and population genetical parameter estimation from sequencing data. Bioinformatics. 27, 2987-2993 (2011).

18. A. R. Quinlan, I. M. Hall, BEDTools: a flexible suite of utilities for comparing genomic features. Bioinformatics. 26, 841-842 (2010).

19. Y. Wu, P. R. Bhat, T. J. Close, S. Lonardi, Efficient and Accurate Construction of Genetic Linkage Maps from the Minimum Spanning Tree of a Graph. PLoS Genet. 4, e1000212 (2008).

20. K. W. Broman, H. Wu, S. Sen, G. A. Churchill, R/qtl: QTL mapping in experimental crosses. Bioinformatics. 19, 889-890 (2003).

21. F. Cabanettes, C. Klopp, D-GENIES: dot plot large genomes in an interactive, efficient and simple way. PeerJ. 6, e4958 (2018).

22. X. Zheng, D. Levine, J. Shen, S. M. Gogarten, C. Laurie, B. S. Weir, A high-performance computing toolset for relatedness and principal component analysis of SNP data. Bioinformatics. 28, 3326-3328 (2012).

23. E. Frichot, F. Mathieu, T. Trouillon, G. Bouchard, O. François, Fast and Efficient Estimation of Individual Ancestry Coefficients. Genetics. 196, 973-983 (2014). 
24. S. R. Browning, B. L. Browning, Rapid and Accurate Haplotype Phasing and Missing-Data Inference for Whole-Genome Association Studies By Use of Localized Haplotype Clustering. Am. J. Hum. Genet. 81, 1084-1097 (2007).

25. G. A. Watterson, On the number of segregating sites in genetical models without recombination. Theor. Popul. Biol. 7, 256-276 (1975).

26. M. Nei, F. Tajima, DNA polymorphism detectable by restriction endonucleases. Genetics. 97, 145-167 (1981).

27. F. Tajima, Statistical method for testing the neutral mutation hypothesis by DNA polymorphism. Genetics. 123, 585-595 (1989).

28. N. Scarcelli, P. Cubry, R. Akakpo, A. C. Thuillet, J. Obidiegwu, M. N. Baco, E. Otoo, B. Sonké, A. Dansi, G. Djedatin, C. Mariac, M. Couderc, S. Causse, K. Alix, H. Chaïr, O. François, Y. Vigouroux, Yam genomics supports West Africa as a major cradle of crop domestication. Sci. Adv. 5, eaaw1947 (2019).

29. N. Saitou, M Nei, The neighbor-joining method: a new method for reconstructing phylogenetic trees. Mol. Biol. Evol. 4, 406-425 (1987).

30. S. Kumar, G. Stecher, M. Li, C. Knyaz, K. Tamura, MEGA X: Molecular Evolutionary Genetics Analysis across Computing Platforms. Mol. Biol. Evol. 35, 1547-1549 (2018).

31. G. Yu, D. K. Smith, H. Zhu, Y. Guan, T. T. Lam, GGTREE: an R package for visualization and annotation of phylogenetic trees with their covariates and other associated data. Methods Ecol. Evol. 8, 28-36 (2017).

32. R. N. Gutenkunst, R. D. Hernandez, S. H. Williamson, C. D. Bustamante, Inferring the Joint Demographic History of Multiple Populations from Multidimensional SNP Frequency Data. PLoS Genet. 5, e1000695 (2009).

33. L. Excoffier, I. Dupanloup, E. Huerta-Sánchez, V. C. Sousa, M. Foll, Robust demographic Inference from genomic and SNP data. PLoS Genet. 9, e1003905 (2013).

34. S. Wright, The genetical structure of populations. Ann. Eugen. 15, 323-354 (1951).

35. H. J. Bandelt, P. Forster, A. Röhl, Median-joining networks for inferring intraspecific phylogenies. Mol. Biol. Evol. 16, 37-48 (1999).

36. J. W. Leigh, D. Bryant, popart: full-feature software for haplotype network construction. Methods Ecol. Evol. 6, 1110-1116 (2015).

37. D. Reich, K. Thangaraj, N. Patterson, A. L. Price, L. Singh, Reconstructing Indian population history. Nature. 461, 489-494 (2009).

38. B. M. Peter, Admixture, Population Structure, and F-Statistics. Genetics. 202, 1485-1501 (2016).

39. D. H. Huson, D. Bryant, Application of Phylogenetic Networks in Evolutionary Studies. Mol. Biol. Evol. 23, 254-267 (2006). 
Table S1. All sequence information of Guinea yam accessions

\begin{tabular}{|c|c|c|c|c|c|c|c|c|c|c|c|}
\hline \multicolumn{3}{|c|}{ Sample } & \multicolumn{2}{|c|}{ Fastq size } & \multicolumn{4}{|c|}{ Aligned bam information } & \multirow[b]{2}{*}{ Sequence platform } & \multirow[b]{2}{*}{ Cluster } & \multirow[b]{2}{*}{ Accession No. } \\
\hline Name & IITA name & Ploidy level & $\begin{array}{c}\text { Original } \\
\text { (Gbp) }\end{array}$ & $\begin{array}{l}\text { Filtered } \\
\text { (Gbp) }\end{array}$ & $\begin{array}{c}\text { Aligned } \\
\text { (Gbp) }\end{array}$ & $\begin{array}{l}\text { Unmapped } \\
\text { (Gbp) }\end{array}$ & $\begin{array}{c}\text { Coverage } \\
(\%)\end{array}$ & Depth & & & \\
\hline TDr04_219 & TDr04_219 & - & 38.26 & 33.10 & 25.95 & 0.32 & 89.7 & 49.93 & MiSeq, HiSeq4000, GAllx & - & DRR208404,DRR208405,DRR063085 \\
\hline TDr97_777 & TDr97_777 & - & 50.20 & 43.48 & 32.72 & 0.94 & 89.8 & 62.90 & MiSeq,HiSeq4000,NextSeq500,GAllx & - & DRR063127,DRR208406,DRR045130-7,DRR063111 \\
\hline TDr96_F1 & TDr96_F1 & - & 16.77 & 21.34 & 17.66 & 0.04 & 90.3 & 33.74 & Miseq & - & DRR027644 \\
\hline DRS_001 & TDr2946A & 2 & 12.70 & 11.32 & 9.28 & 0.12 & 86.4 & 18.53 & HiSeq4000 & - & DRR208876 \\
\hline DRS_002 & TDr1489A & 2 & 8.09 & 7.02 & 5.27 & 0.05 & 79.0 & 11.51 & HiSeq4000 & - & DRR208761 \\
\hline DRS_003 & TDr2284A & 2 & 15.28 & 13.18 & 9.50 & 0.16 & 87.3 & 18.78 & HiSeq4000,NextSeq500 & - & DRR208762,DRR208884 \\
\hline DRS_004 & TDr1499A & 2 & 13.65 & 11.70 & 8.55 & 0.13 & 86.9 & 16.99 & HiSeq4000,NextSeq500 & cluster2 & DRR208763,DRR208885 \\
\hline DRS_006 & TDr1509A & 2 & 13.47 & 11.10 & 8.13 & 0.16 & 86.6 & 16.19 & HiSeq4000,NextSeq500 & - & DRR208764,DRR208886 \\
\hline DRS_007 & TDr1510A & 2 & 12.30 & 10.19 & 7.81 & 0.14 & 87.2 & 15.46 & HiSeq4000,NextSeq500 & - & DRR208765,DRR208887 \\
\hline DRS_009 & TDr3782A & 2 & 13.23 & 11.21 & 7.62 & 0.14 & 89.0 & 14.77 & HiSeq4000,NextSeq500 & - & DRR208766,DRR208888 \\
\hline DRS_010 & TDr1533A & 2 & 13.31 & 11.18 & 8.32 & 0.17 & 86.6 & 16.58 & HiSeq4000,NextSeq500 & cluster3 & DRR208767,DRR208889 \\
\hline DRS_011 & TDr1543A & 2 & 13.22 & 11.17 & 7.33 & 0.18 & 86.7 & 14.59 & HiSeq4000,NextSeq500 & cluster3 & DRR208768,DRR208890 \\
\hline DRS_012 & TDr1858C & 2 & 14.22 & 12.56 & 9.99 & 0.11 & 85.6 & 20.14 & HiSeq4000 & - & DRR208877 \\
\hline DRS_013 & TDr1576A & 2 & 12.81 & 11.47 & 9.91 & 0.15 & 87.4 & 19.57 & HiSeq4000,NextSeq500 & cluster3 & DRR208769,DRR208891 \\
\hline DRS_014 & TDr1585A & 2 & 14.01 & 11.78 & 7.58 & 0.15 & 87.1 & 15.02 & HiSeq4000,NextSeq500 & - & DRR208770,DRR208892 \\
\hline DRS_015 & TDr1585C & 2 & 15.02 & 12.78 & 8.02 & 0.16 & 87.0 & 15.92 & HiSeq4000,NextSeq500 & cluster2 & DRR208771,DRR208893 \\
\hline DRS_016 & TDr1598A & 3 & 13.80 & 11.74 & 7.54 & 0.43 & 86.6 & 15.02 & HiSeq4000,NextSeq500 & cluster1 & DRR208772,DRR208894 \\
\hline DRS_017 & TDr1622A & 2 & 12.71 & 10.81 & 7.02 & 0.15 & 86.8 & 13.96 & HiSeq4000,NextSeq500 & cluster2 & DRR208773,DRR208895 \\
\hline DRS_018 & TDr1628A & 2 & 8.08 & 7.00 & 5.24 & 0.05 & 77.7 & 11.65 & HiSeq4000 & - & DRR208774 \\
\hline DRS_019 & TDr1631C & 2 & 15.29 & 13.62 & 11.34 & 0.17 & 87.8 & 22.31 & HiSeq4000,NextSeq500 & - & DRR208775,DRR208896 \\
\hline DRS_020 & TDr1649A & 2 & 12.64 & 10.83 & 7.22 & 0.15 & 86.4 & 14.42 & HiSeq4000,NextSeq500 & cluster3 & DRR208776,DRR208897 \\
\hline DRS_021 & TDr1650B & 2 & 12.42 & 10.56 & 8.18 & 0.15 & 87.0 & 16.23 & HiSeq4000,NextSeq500 & cluster2 & DRR208777,DRR208898 \\
\hline DRS_022 & TDr1653A & 2 & 12.68 & 10.86 & 6.92 & 0.13 & 86.5 & 13.80 & HiSeq4000,NextSeq500 & - & DRR208778,DRR208899 \\
\hline DRS_023 & TDr1655A & 2 & 12.24 & 10.36 & 6.42 & 0.16 & 86.0 & 12.89 & HiSeq4000,NextSeq500 & cluster5 & DRR208779,DRR208900 \\
\hline DRS_024 & TDr1663A & 2 & 13.69 & 11.55 & 8.68 & 0.17 & 86.9 & 17.22 & HiSeq4000,NextSeq500 & - & DRR208780,DRR208901 \\
\hline DRS_025 & TDr1686A & 2 & 12.58 & 11.14 & 8.11 & 0.12 & 88.2 & 15.87 & HiSeq4000,NextSeq500 & cluster4 & DRR208781,DRR208902 \\
\hline DRS_026 & TDr1707A & 2 & 13.51 & 11.93 & 9.04 & 0.15 & 88.7 & 17.58 & HiSeq4000,NextSeq500 & - & DRR208782,DRR208903 \\
\hline DRS_027 & TDr1709A & 2 & 16.91 & 15.10 & 12.04 & 0.22 & 86.1 & 24.13 & HiSeq4000 & - & DRR208878 \\
\hline DRS_028 & TDr1711A & 2 & 7.64 & 6.65 & 5.15 & 0.04 & 80.3 & 11.06 & HiSeq4000 & - & DRR208783 \\
\hline DRS_029 & TDr3872A & 2 & 12.61 & 11.23 & 8.95 & 0.15 & 87.1 & 17.73 & HiSeq4000,NextSeq500 & - & DRR208784,DRR208904 \\
\hline DRS_030 & TDr1732A & 2 & 13.95 & 12.38 & 9.83 & 0.16 & 88.2 & 19.23 & HiSeq4000,NextSeq500 & cluster4 & DRR208785,DRR208905 \\
\hline DRS_031 & TDr1735A & 2 & 13.02 & 11.59 & 7.48 & 0.15 & 86.5 & 14.93 & HiSeq4000,NextSeq500 & - & DRR208786,DRR208906 \\
\hline DRS_032 & TDr2029A & 2 & 11.77 & 10.51 & 7.03 & 0.14 & 85.7 & 14.16 & HiSeq4000,NextSeq500 & - & DRR208787,DRR208907 \\
\hline DRS_033 & TDr1760A & 2 & 11.42 & 9.96 & 8.19 & 0.08 & 87.9 & 16.09 & HiSeq4000,NextSeq500 & cluster4 & DRR208788,DRR208908 \\
\hline DRS_034 & TDr1763C & 2 & 16.32 & 14.06 & 11.22 & 0.09 & 87.7 & 22.07 & HiSeq4000,NextSeq500 & cluster4 & DRR208789,DRR208909 \\
\hline DRS_035 & TDr1804A & 2 & 12.63 & 10.98 & 8.56 & 0.10 & 87.7 & 16.85 & HiSeq4000,NextSeq500 & cluster4 & DRR208790,DRR208910 \\
\hline DRS_036 & TDr1775A & 3 & 11.44 & 9.96 & 7.52 & 0.27 & 87.5 & 14.82 & HiSeq4000,NextSeq500 & cluster1 & DRR208791,DRR208911 \\
\hline DRS_037 & TDr1798A & 2 & 8.14 & 7.02 & 5.25 & 0.05 & 79.8 & 11.35 & HiSeq4000 & - & DRR208792 \\
\hline DRS_038 & TDr1805A & 2 & 12.60 & 11.14 & 9.16 & 0.08 & 86.7 & 18.22 & HiSeq4000,NextSeq500 & - & DRR208793,DRR208912 \\
\hline DRS_039 & TDr1807A & 3 & 11.35 & 10.05 & 7.67 & 0.26 & 87.6 & 15.11 & HiSeq4000,NextSeq500 & cluster1 & DRR208794,DRR208913 \\
\hline DRS_040 & TDr1829A & 2 & 11.26 & 9.77 & 7.35 & 0.10 & 85.9 & 14.77 & HiSeq4000,NextSeq500 & cluster3 & DRR208795,DRR208914 \\
\hline DRS_041 & TDr1850A & 2 & 11.35 & 10.03 & 8.20 & 0.09 & 86.5 & 16.36 & HiSeq4000,NextSeq500 & cluster5 & DRR208796,DRR208915 \\
\hline DRS_042 & TDr1899A & 2 & 13.90 & 12.01 & 7.89 & 0.15 & 86.8 & 15.68 & HiSeq4000,NextSeq500 & - & DRR208797,DRR208916 \\
\hline DRS_043 & TDr1922C & 2 & 7.43 & 6.29 & 4.76 & 0.05 & 79.7 & 10.31 & HiSeq4000 & - & DRR208798 \\
\hline DRS_044 & TDr1935A & 2 & 11.67 & 10.14 & 7.65 & 0.14 & 86.7 & 15.23 & HiSeq4000,NextSeq500 & - & DRR208799,DRR208917 \\
\hline DRS_045 & TDr2608A & 2 & 12.53 & 10.86 & 7.99 & 0.18 & 86.7 & 15.91 & HiSeq4000,NextSeq500 & - & DRR208800,DRR208918 \\
\hline DRS_046 & TDr2041B & 2 & 14.19 & 12.52 & 10.16 & 0.16 & 86.6 & 20.26 & HiSeq4000,NextSeq500 & - & DRR208801,DRR208919 \\
\hline
\end{tabular}




\begin{tabular}{|c|c|c|c|c|c|c|}
\hline DRS_047 & TDr2121A & 2 & 11.61 & 10.17 & 7.98 & 0.10 \\
\hline DRS_048 & TDr2155A & 3 & 13.17 & 11.47 & 7.84 & 0.41 \\
\hline DRS_049 & TDr2159A & 2 & 11.28 & 9.93 & 7.79 & 0.20 \\
\hline DRS_050 & TDr2161C & 3 & 12.97 & 11.43 & 7.79 & 0.42 \\
\hline DRS_051 & TDr2167A & 3 & 11.86 & 10.44 & 7.60 & 0.36 \\
\hline DRS_053 & TDr2207A & 2 & 11.41 & 9.81 & 7.75 & 0.08 \\
\hline DRS_054 & TDr2210A & 2 & 10.71 & 9.52 & 7.15 & 0.14 \\
\hline DRS_055 & TDr3311B & 2 & 13.88 & 12.29 & 9.69 & 0.18 \\
\hline DRS_056 & TDr2262C & 2 & 11.63 & 10.36 & 8.70 & 0.19 \\
\hline DRS_057 & TDr2320A & 2 & 8.95 & 7.70 & 6.41 & 0.08 \\
\hline DRS_058 & TDr2484A & 2 & 12.62 & 11.21 & 9.03 & 0.13 \\
\hline DRS_059 & TDr2973A & 2 & 11.15 & 9.46 & 7.31 & 0.10 \\
\hline DRS_060 & TDr2425B & 2 & 10.38 & 8.92 & 7.47 & 0.07 \\
\hline DRS_061 & TDr2427B & 3 & 12.28 & 11.17 & 8.47 & 0.38 \\
\hline DRS_062 & TDr2435A & 2 & 7.61 & 6.72 & 5.79 & 0.05 \\
\hline DRS_063 & TDr2439A & 2 & 10.11 & 9.03 & 7.64 & 0.06 \\
\hline DRS_064 & TDr2453A & 2 & 13.41 & 12.08 & 9.89 & 0.16 \\
\hline DRS_065 & TDr2491A & 2 & 13.74 & 12.46 & 10.23 & 0.15 \\
\hline DRS_066 & TDr3569A & 2 & 15.47 & 14.08 & 10.11 & 0.15 \\
\hline DRS_067 & TDr2533C & 2 & 11.24 & 10.03 & 7.83 & 0.12 \\
\hline DRS_068 & TDr2554A & 3 & 16.37 & 14.91 & 9.73 & 0.57 \\
\hline DRS_069 & TDr2575A & 2 & 8.89 & 8.03 & 7.04 & 0.09 \\
\hline DRS_070 & TDr2636B & 2 & 8.88 & 7.89 & 6.48 & 0.09 \\
\hline DRS_071 & TDr2674A & 2 & 8.56 & 7.72 & 6.81 & 0.07 \\
\hline DRS_072 & TDr2713A & 2 & 13.04 & 11.77 & 9.86 & 0.13 \\
\hline DRS_073 & TDr1684A & 2 & 15.55 & 13.87 & 11.48 & 0.08 \\
\hline DRS_074 & TDr2948A & 2 & 11.67 & 10.50 & 8.98 & 0.07 \\
\hline DRS_075 & TDr2965A & 2 & 12.16 & 10.98 & 9.64 & 0.10 \\
\hline DRS_076 & TDr2968A & 2 & 9.19 & 8.21 & 6.80 & 0.09 \\
\hline DRS_077 & TDr2975A & 2 & 11.13 & 9.98 & 8.47 & 0.08 \\
\hline DRS_078 & TDr4067A & 3 & 12.84 & 11.59 & 8.41 & 0.43 \\
\hline DRS_079 & TDr2577A & 2 & 13.00 & 11.79 & 8.57 & 0.14 \\
\hline DRS_080 & TDr3325A & 2 & 13.81 & 12.10 & 10.14 & 0.19 \\
\hline DRS_081 & TDr3470A & 2 & 9.57 & 8.49 & 6.66 & 0.11 \\
\hline DRS_082 & TDr3436A & 2 & 9.71 & 6.42 & 5.15 & 0.05 \\
\hline DRS_083 & TDr3447B & 2 & 12.45 & 9.46 & 6.69 & 0.11 \\
\hline DRS_084 & TDr3519A & 3 & 16.08 & 14.55 & 9.55 & 0.49 \\
\hline DRS_085 & TDr3527A & 2 & 7.58 & 6.63 & 5.61 & 0.05 \\
\hline DRS_086 & TDr2276A & 2 & 15.07 & 13.13 & 9.37 & 0.16 \\
\hline DRS_087 & TDr3576A & 2 & 17.05 & 13.22 & 10.14 & 0.16 \\
\hline DRS_088 & TDr3624B & 2 & 9.68 & 7.84 & 6.21 & 0.09 \\
\hline DRS_089 & TDr2503A & 2 & 10.12 & 9.07 & 7.50 & 0.09 \\
\hline DRS_090 & TDr3678A & 2 & 15.57 & 13.52 & 9.08 & 0.16 \\
\hline DRS_091 & TDr3719A & 2 & 10.51 & 8.83 & 7.13 & 0.12 \\
\hline DRS_092 & TDr3828B & 2 & 14.56 & 13.01 & 9.32 & 0.14 \\
\hline DRS_093 & TDr3842A & 2 & 16.92 & 14.92 & 12.90 & 0.15 \\
\hline DRS_094 & TDr3863A & 2 & 12.26 & 10.95 & 9.22 & 0.08 \\
\hline DRS_095 & TDr3955C & 2 & 12.58 & 11.25 & 9.49 & 0.13 \\
\hline DRS_096 & TDr2090B & 2 & 11.97 & 10.73 & 8.40 & 0.14 \\
\hline DRS_097 & TDr1772A & 2 & 11.77 & 10.45 & 7.60 & 0.17 \\
\hline DRS 098 & TDr3357A & 2 & 12.18 & 10.91 & 9.10 & 0.14 \\
\hline
\end{tabular}

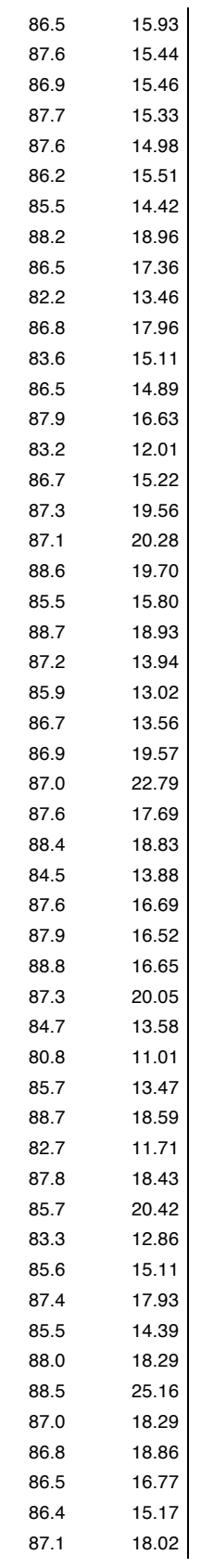

HiSeq4000,NextSeq500

HiSeq4000, NextSeq500

HiSeq4000, NextSeq50

HiSeq4000,NextSeq500

HiSeq4000,NextSeq500$$
\text { HiSeq4000 }
$$

HiSeq4000,NextSeq500

HiSeq4000,NextSeq500

HiSeq4000,NextSeq500

HiSeq4000,NextSeq500

HiSeq4000,NextSeq50

HiSeq4000,NextSeq500$$
\text { Hiseq4000 }
$$

HiSeq4000,NextSeq500

HiSeq4000,NextSeq500

HiSeq4000,NextSeq50

$$
\text { HiSeq4000 }
$$

HiSeq4000,NextSeq50

HiSeq4000,NextSeq500

HiSeq4000,NextSeq500

HiSeq4000,NextSeq500

HiSeq4000,NextSeq500

HiSeq4000,NextSeq500

HiSeq4000,NextSeq500

HiSeq4000,NextSeq500$$
\text { HiSeq4000 }
$$

HiSeq4000, NextSeq500

HiSeq4000, NextSeg500

HiSeq4000,NextSeq500

Hiseq4000,NextSeq500

$$
\text { Hiseq4000 }
$$

HiSeq4000,NextSeq500

HiSeq4000,NextSeq500

HiSeq4000,NextSeq500

$$
\text { Hiseq4000 }
$$

HiSeq4000 NextSeq500

HiSeq4000,NextSeq500

HiSeq4000, NextSeq500 HiSeq4000

4000, NextSeg500

HiSeq4000,NextSeq500

HiSeq4000,NextSeq500

HiSeq4000, NextSeq500

HiSeq4000,NextSeq500

HiSeq4000,NextSeq500

HiSeq4000,NextSeq500

HiSeq4000,NextSeq500 cluster3

cluster

cluster1

cluster2

cluster4

cluster3

cluster1

cluster3

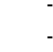

cluster

cluster3

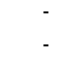

clust

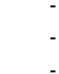

cluster4

cluster

cluster3

cluster1

cluster

cluster 5
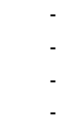

cluster5

cluster2
DRR208802 DRR208920 RR20892 RR208804,DRR208922 DRR208805,DRR208923 DRR208806,DRR208924 DRR208807,DRR20892 DRR208879

DRR208808,DRR208926 RR208809,DRR208927 DRR208928

DRR208810,DRR208929 DRR208930 DRR208931

DRR208811,DRR208932 DRR208812

DRR208813,DRR208933

DRR208814,DRR208934

DRR208815,DRR208935

DRR208816,DRR208936 DRR208880

DRR208817. DRR208937 DRR208938 DRR208939 DRR208940

DRR208818,DRR20894 DRR208819,DRR208942 DRR208820,DRR208943 DRR208944 DRR20888

DRR208821,DRR208946 DRR208822,DRR208947 DRR208823,DRR208948 DRR208882

DRR208824,DRR208949 DRR208825,DRR208950 DRR208826,DRR20895 DRR208827 DRR208953 DRR208954 R208829,DRR208955 DRR208956

DRR208830,DRR208957 DRR208958

DRR208831,DRR208959 DRR208832,DRR208960

DRR208833,DRR208961

DRR208834,DRR208962

DRR208835,DRR208963 


\begin{tabular}{|c|c|c|c|c|c|c|c|c|c|c|c|}
\hline DRS_099 & TDr4017A & 2 & 13.05 & 11.46 & 8.35 & 0.21 & 86.8 & 16.59 & HiSeq4000,NextSeq500 & cluster3 & DRR208836,DRR208964 \\
\hline DRS_100 & TDr3623C & 2 & 13.73 & 11.91 & 8.88 & 0.19 & 87.8 & 17.45 & HiSeq 4000, NextSeq500 & cluster4 & DRR208837,DRR208965 \\
\hline DRS_101 & TDr4100A & 2 & 13.31 & 11.67 & 9.37 & 0.18 & 87.1 & 18.57 & HiSeq 4000, NextSeq500 & cluster5 & DRR208838,DRR208966 \\
\hline DRS_102 & TDr2826A & 2 & 8.63 & 7.53 & 6.33 & 0.05 & 83.7 & 13.06 & HiSeq4000 & - & DRR208839 \\
\hline DRS_103 & TDr4155A & 2 & 10.98 & 9.61 & 7.67 & 0.15 & 87.4 & 15.14 & HiSeq 4000, NextSeq500 & - & DRR208840,DRR208967 \\
\hline DRS_104 & TDr4180A & 2 & 11.13 & 9.80 & 7.41 & 0.17 & 86.1 & 14.87 & HiSeq 4000, NextSeq500 & cluster5 & DRR208841,DRR208968 \\
\hline DRS_106 & TDr2042A & 2 & 11.49 & 10.05 & 7.77 & 0.14 & 86.4 & 15.53 & HiSeq 4000, NextSeq500 & - & DRR208842,DRR208969 \\
\hline DRS_165 & TDr608 & - & 10.92 & 9.99 & 7.98 & 0.04 & 86.1 & 15.99 & HiSeq4000 & - & DRR208843 \\
\hline DRS_169 & TDrFaketsa & - & 13.01 & 11.98 & 9.39 & 0.07 & 85.2 & 19.00 & HiSeq4000 & - & DRR208844 \\
\hline DRS_177 & TDrGbangu & - & 11.57 & 10.54 & 8.17 & 0.05 & 84.2 & 16.74 & HiSeq4000 & - & DRR208845 \\
\hline DRS_208 & TDrog/00362 & - & 9.01 & 8.25 & 6.58 & 0.04 & 83.7 & 13.57 & HiSeq 4000 & - & DRR208846 \\
\hline DRS_211 & TDro9/00799 & - & 10.73 & 9.84 & 7.82 & 0.04 & 85.3 & 15.83 & HiSeq4000 & - & DRR208847 \\
\hline DRS_212 & TDrMeccakusa & - & 8.41 & 7.64 & 6.18 & 0.03 & 84.4 & 12.64 & HiSeq4000 & - & DRR208848 \\
\hline DRS_213 & TDrog/09132 & - & 9.97 & 9.14 & 7.40 & 0.04 & 85.3 & 14.98 & HiSeq4000 & - & DRR208849 \\
\hline DRS_220 & TDrOjuiyawo & - & 7.58 & 6.97 & 5.80 & 0.04 & 85.7 & 11.69 & HiSeq4000 & - & DRR208850 \\
\hline DRS_253 & TDr2119 & - & 9.33 & 8.57 & 7.03 & 0.05 & 84.6 & 14.34 & HiSeq4000 & cluster2 & DRR208851 \\
\hline DRS_259 & TDr2347 & - & 9.94 & 9.10 & 6.98 & 0.05 & 85.1 & 14.16 & HiSeq4000 & cluster4 & DRR208852 \\
\hline DRS_282 & TDrOgoja & - & 12.34 & 11.27 & 8.64 & 0.06 & 85.0 & 17.54 & HiSeq4000 & - & DRR208853 \\
\hline DRS_293 & TDr10/00077 & - & 10.65 & 9.72 & 7.73 & 0.05 & 83.1 & 16.05 & HiSeq4000 & - & DRR208854 \\
\hline DRS_297 & TDrGbongi & - & 9.56 & 8.63 & 6.51 & 0.06 & 84.2 & 13.35 & HiSeq4000 & - & DRR208855 \\
\hline DRS_307 & TDr10/00125 & - & 8.97 & 8.23 & 6.41 & 0.04 & 83.3 & 13.27 & HiSeq4000 & - & DRR208856 \\
\hline DRS_312 & TDrLagos & - & 7.85 & 7.13 & 5.63 & 0.05 & 82.8 & 11.73 & HiSeq4000 & - & DRR208857 \\
\hline DRS_318 & TDrHembakwase & - & 9.25 & 8.48 & 6.86 & 0.05 & 85.6 & 13.84 & HiSeq4000 & - & DRR208858 \\
\hline DRS_320 & TDr89/02157 & - & 11.44 & 10.42 & 8.06 & 0.05 & 85.4 & 16.30 & HiSeq4000 & - & DRR208859 \\
\hline DRS_322 & TDr97/00632 & - & 8.64 & 7.92 & 6.19 & 0.05 & 82.3 & 12.98 & HiSeq4000 & - & DRR208860 \\
\hline DRS_324 & TDroo/02405 & - & 11.07 & 10.00 & 7.70 & 0.05 & 84.4 & 15.74 & HiSeq4000 & - & DRR208861 \\
\hline DRS_325 & TDr10/00013 & - & 10.25 & 9.28 & 7.26 & 0.06 & 84.0 & 14.90 & HiSeq4000 & - & DRR208862 \\
\hline DRS_326 & TDr10/00048 & - & 8.99 & 8.28 & 6.81 & 0.04 & 84.6 & 13.90 & HiSeq4000 & - & DRR208863 \\
\hline DRS_327 & TDr10/00179 & - & 9.13 & 8.29 & 6.55 & 0.05 & 83.5 & 13.53 & HiSeq4000 & - & DRR208864 \\
\hline DRS_328 & TDr10/00344 & - & 10.16 & 9.27 & 7.49 & 0.04 & 84.8 & 15.25 & HiSeq4000 & - & DRR208865 \\
\hline DRS_329 & TDr10/00360 & - & 11.47 & 10.35 & 8.04 & 0.05 & 84.6 & 16.41 & HiSeq4000 & - & DRR208866 \\
\hline DRS_330 & TDr10/00459 & - & 11.39 & 10.42 & 8.27 & 0.05 & 84.3 & 16.93 & HiSeq4000 & - & DRR208867 \\
\hline DRS_331 & TDr10/00021 & - & 10.88 & 9.96 & 8.05 & 0.05 & 85.6 & 16.24 & HiSeq4000 & - & DRR208868 \\
\hline DRS_332 & TDr89/02475 & - & 7.70 & 7.05 & 5.97 & 0.04 & 85.5 & 12.05 & HiSeq4000 & - & DRR208869 \\
\hline DRS_333 & TDr89/02677 & - & 9.64 & 8.89 & 7.41 & 0.05 & 85.9 & 14.89 & HiSeq4000 & - & DRR208870 \\
\hline DRS_334 & TDr96/00629 & - & 9.43 & 8.64 & 6.94 & 0.04 & 86.3 & 13.88 & HiSeq4000 & - & DRR208871 \\
\hline DRS_335 & TDr96/01818 & - & 10.27 & 9.39 & 7.53 & 0.05 & 86.3 & 15.07 & HiSeq4000 & - & DRR208872 \\
\hline DRS_336 & TDr99/02562 & - & 10.56 & 9.66 & 7.89 & 0.05 & 85.9 & 15.84 & HiSeq4000 & - & DRR208873 \\
\hline DRS_337 & TDrAkwuchi & - & 9.43 & 8.65 & 7.11 & 0.04 & 86.0 & 14.28 & HiSeq4000 & - & DRR208874 \\
\hline DRS_338 & TDrDanacha & - & 10.57 & 9.54 & 7.64 & 0.06 & 84.6 & 15.59 & HiSeq4000 & - & DRR208875 \\
\hline TDr_001 & TDr1492 & - & 8.93 & 7.47 & 6.00 & 0.05 & 81.8 & 12.66 & HiSeq4000 & cluster3 & DRR208563 \\
\hline TDr_002 & TDr2262 & - & 6.84 & 5.83 & 4.90 & 0.04 & 82.5 & 10.24 & HiSeq4000 & - & DRR208564 \\
\hline TDr_003 & TDr1533 & - & 7.61 & 6.25 & 5.00 & 0.05 & 78.6 & 10.98 & HiSeq4000 & cluster3 & DRR208565 \\
\hline TDr_004 & TDr1559 & - & 8.65 & 7.51 & 5.75 & 0.07 & 84.1 & 11.81 & HiSeq4000 & cluster4 & DRR208566 \\
\hline TDr_005 & TDr1577 & - & 8.77 & 7.73 & 6.22 & 0.22 & 81.6 & 13.14 & HiSeq4000 & cluster3 & DRR208567 \\
\hline TDr_006 & TDr1598 & - & 9.47 & 8.18 & 5.96 & 0.07 & 82.6 & 12.44 & HiSeq4000 & cluster2 & DRR208568 \\
\hline TDr_007 & TDr1615 & - & 8.48 & 7.14 & 5.27 & 0.16 & 81.3 & 11.17 & HiSeq4000 & cluster1 & DRR208569 \\
\hline TDr_008 & TDr1628 & - & 7.36 & 6.27 & 5.35 & 0.05 & 84.4 & 10.94 & HiSeq4000 & - & DRR208570 \\
\hline TDr_009 & TDr1669 & - & 7.41 & 6.53 & 4.90 & 0.03 & 81.0 & 10.44 & HiSeq4000 & cluster2 & DRR208571 \\
\hline TDr_010 & TDr1707 & - & 9.48 & 8.20 & 6.20 & 0.06 & 84.4 & 12.68 & HiSeq 4000 & - & DRR208572 \\
\hline TDr_011 & TDr1717 & - & 8.85 & 7.98 & 6.13 & 0.05 & 82.2 & 12.88 & HiSeq4000 & - & DRR208573 \\
\hline
\end{tabular}




\begin{tabular}{|c|c|}
\hline TDr_012 & TDr1763 \\
\hline TDr_013 & TDr1769 \\
\hline TDr_014 & TDr1799 \\
\hline TDr_015 & TDr1825 \\
\hline TDr_016 & TDr1876 \\
\hline TDr_017 & TDr1937 \\
\hline TDr_018 & TDr1939 \\
\hline TDr_019 & TDr1949 \\
\hline TDr_020 & TDr2015 \\
\hline TDr_021 & TDr2028 \\
\hline TDr_022 & TDr2038 \\
\hline TDr_023 & TDr2050 \\
\hline TDr_024 & TDr2059 \\
\hline TDr_025 & TDr2090 \\
\hline TDr_026 & TDr2104 \\
\hline TDr_027 & TDr2110 \\
\hline TDr_028 & TDr2211 \\
\hline TDr_029 & TDr2080 \\
\hline TDr_030 & TDr2349 \\
\hline TDr_031 & TDr2363 \\
\hline TDr_032 & TDr2406 \\
\hline TDr_033 & TDr2432 \\
\hline TDr_034 & TDr2439 \\
\hline TDr_035 & TDr2458 \\
\hline TDr_036 & TDr2502 \\
\hline TDr_037 & TDr2581 \\
\hline TDr_038 & TDr2645 \\
\hline TDr_039 & TDr2674 \\
\hline TDr_040 & TDr2681 \\
\hline TDr_041 & TDr2683 \\
\hline TDr_042 & TDr2687 \\
\hline TDr_043 & TDr2701 \\
\hline TDr_044 & TDr2724 \\
\hline TDr_045 & TDr2694 \\
\hline TDr_046 & TDr2770 \\
\hline TDr_047 & TDr2936 \\
\hline TDr_048 & TDr2965 \\
\hline TDr_049 & TDr2973 \\
\hline TDr_050 & TDr3002 \\
\hline TDr_051 & TDr09/00064 \\
\hline TDr_052 & TDro0/00362 \\
\hline TDr_053 & TDr05/00589 \\
\hline TDr_054 & TDr05/00632 \\
\hline TDr_055 & TDro7/00157 \\
\hline TDr_056 & TDro9/01932 \\
\hline TDr_057 & TDr08/00092 \\
\hline TDr_058 & TDro8/00108 \\
\hline TDr_059 & TDr08/00122 \\
\hline TDr_061 & TDr07/00732 \\
\hline TDr_062 & TDr08/00207 \\
\hline TDr_063 & TDr08/00617 \\
\hline
\end{tabular}

\begin{tabular}{rr|rlrr}
8.62 & 7.76 & 6.09 & 0.05 & 82.2 & 12.79 \\
10.00 & 8.55 & 6.64 & 0.23 & 85.9 & 13.34 \\
7.81 & 6.87 & 4.65 & 0.03 & 80.1 & 10.02 \\
8.01 & 6.33 & 5.14 & 0.05 & 82.8 & 10.71 \\
9.56 & 8.32 & 6.48 & 0.21 & 85.7 & 13.06 \\
10.02 & 8.61 & 6.82 & 0.06 & 82.5 & 14.27 \\
9.87 & 8.09 & 6.57 & 0.05 & 83.1 & 13.64 \\
8.22 & 7.20 & 6.01 & 0.05 & 82.3 & 12.60 \\
8.50 & 7.36 & 5.69 & 0.17 & 84.8 & 11.58 \\
9.44 & 8.27 & 6.56 & 0.21 & 86.1 & 13.16 \\
7.88 & 6.63 & 5.50 & 0.03 & 83.7 & 11.35 \\
10.79 & 8.75 & 6.75 & 0.08 & 81.8 & 14.23 \\
10.16 & 8.59 & 6.95 & 0.06 & 85.6 & 14.03 \\
7.64 & 6.44 & 4.68 & 0.05 & 80.0 & 10.10 \\
8.55 & 7.51 & 5.58 & 0.06 & 82.4 & 11.69 \\
9.84 & 8.65 & 6.78 & 0.21 & 85.7 & 13.66 \\
9.65 & 8.28 & 6.96 & 0.05 & 84.9 & 14.16 \\
9.78 & 8.47 & 7.43 & 0.07 & 86.4 & 14.86 \\
9.61 & 8.37 & 7.24 & 0.12 & 87.1 & 14.36 \\
7.70 & 6.26 & 4.78 & 0.04 & 79.6 & 10.37 \\
10.33 & 9.01 & 7.83 & 0.06 & 85.2 & 15.86 \\
6.70 & 5.57 & 4.63 & 0.04 & 81.6 & 9.80 \\
9.57 & 7.96 & 6.11 & 0.06 & 82.3 & 12.80 \\
6.94 & 5.83 & 5.02 & 0.04 & 84.7 & 10.24 \\
6.51 & 5.58 & 4.40 & 0.04 & 80.6 & 9.43 \\
9.62 & 8.34 & 7.10 & 0.10 & 86.3 & 14.20 \\
9.37 & 8.43 & 6.38 & 0.05 & 82.1 & 13.41 \\
7.65 & 6.59 & 5.29 & 0.04 & 82.2 & 11.10 \\
10.16 & 8.00 & 5.62 & 0.18 & 82.6 & 11.74 \\
9.63 & 6.47 & 4.97 & 0.09 & 78.7 & 10.89 \\
14.48 & 12.64 & 11.02 & 0.10 & 85.8 & 22.16 \\
9.63 & 7.79 & 6.41 & 0.06 & 84.9 & 13.03 \\
10.14 & 6.15 & 4.76 & 0.10 & 81.4 & 10.10 \\
8.06 & 7.00 & 6.09 & 0.05 & 84.8 & 12.40 \\
9.33 & 7.46 & 5.42 & 0.07 & 82.0 & 11.40 \\
10.09 & 8.13 & 5.54 & 0.09 & 80.8 & 11.83 \\
10.01 & 8.76 & 7.15 & 0.06 & 82.9 & 14.90 \\
13.14 & 11.33 & 8.88 & 0.28 & 86.9 & 17.64 \\
9.89 & 7.16 & 5.32 & 0.08 & 79.7 & 11.52 \\
8.52 & 5.64 & 4.47 & 0.07 & 78.5 & 9.82 \\
8.13 & 7.27 & 6.03 & 0.05 & 84.4 & 12.32 \\
12.86 & 11.09 & 9.63 & 0.08 & 85.1 & 19.53 \\
7.87 & 6.74 & 5.29 & 0.06 & 80.3 & 11.37 \\
8.49 & 7.10 & 6.10 & 0.05 & 83.8 & 12.57 \\
8.47 & 7.64 & 6.48 & 0.05 & 84.9 & 13.16 \\
8.19 & 6.98 & 5.69 & 0.08 & 81.2 & 12.08 \\
9.46 & 8.61 & 7.02 & 0.06 & 85.2 & 14.21 \\
8.98 & 8.09 & 6.63 & 0.09 & 85.1 & 13.44 \\
10.10 & 9.16 & 7.79 & 0.06 & 85.1 & 15.80 \\
8.83 & 7.27 & 6.13 & 0.07 & 84.7 & 12.49 \\
9.35 & 8.46 & 7.02 & 0.06 & 84.6 & 14.32
\end{tabular}

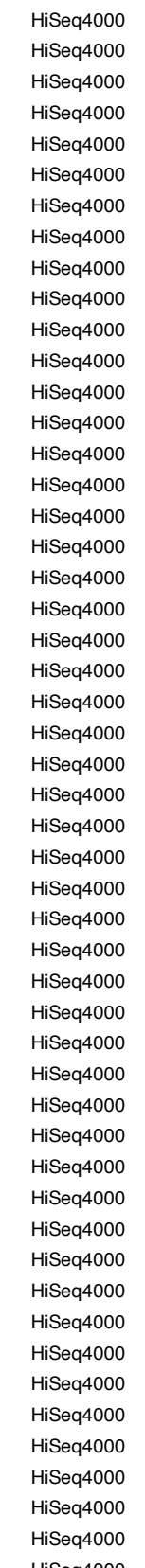

\begin{tabular}{cc} 
& \\
cluster1 & DRR208574 \\
- & DRR208575 \\
cluster4 & DRR208576 \\
cluster1 & DRR208577 \\
cluster3 & DRR208578 \\
cluster2 & DRR208579 \\
cluster2 & DRR208580 \\
cluster1 & DRR208581 \\
cluster1 & DRR208582 \\
- & DRR208583 \\
- & DRR208584 \\
cluster4 & DRR208585 \\
- & DRR208586 \\
cluster4 & DRR208587 \\
cluster1 & DRR208588 \\
- & DRR208589 \\
- & DRR208590 \\
- & DRR208591 \\
cluster2 & DRR208592 \\
cluster2 & DRR208593 \\
cluster2 & DRR208594 \\
- & DRR208595 \\
cluster4 & DRR208596 \\
cluster5 & DRR208597 \\
cluster4 & DRR208598 \\
cluster3 & DRR208599 \\
cluster5 & DRR208600 \\
cluster1 & DRR208601 \\
cluster3 & DRR208602 \\
cluster3 & DRR208603 \\
- & DRR208604 \\
- & DRR208605 \\
cluster2 & DRR208606 \\
cluster4 & DRR208607 \\
- & DRR208608 \\
- & DRR208609 \\
cluster1 & DRR208610 \\
cluster3 & DRR208611 \\
- & DRR208612 \\
- & DRR208613 \\
- & DRR208614 \\
- & DRR208615 \\
- & DRR208616 \\
- & DRR208617 \\
- & DRR208618 \\
- & DRR208619 \\
- & DRR208620 \\
- & DRR208621 \\
- & DRR208622 \\
- & DRR208623 \\
- & DRR208624 \\
\hline & \\
& \\
\hline
\end{tabular}




\begin{tabular}{|c|c|c|c|c|c|c|c|c|c|}
\hline TDr_064 & TDr08/00799 & - & 11.50 & 10.44 & 8.23 & 0.55 & 85.7 & 16.58 & HiSeq4000 \\
\hline TDr_065 & TDr09/00325 & - & 14.08 & 12.80 & 10.42 & 0.09 & 85.9 & 20.96 & HiSeq4000 \\
\hline TDr_066 & TDr96/02433 & - & 14.54 & 13.18 & 10.40 & 0.15 & 85.7 & 20.95 & HiSeq4000 \\
\hline TDr_067 & TDr08/01344 & - & 15.31 & 14.04 & 11.58 & 0.10 & 86.3 & 23.16 & HiSeq4000 \\
\hline TDr_068 & TDr08/01024 & - & 6.51 & 5.79 & 4.89 & 0.04 & 84.5 & 9.99 & HiSeq4000 \\
\hline TDr_069 & TDr09/00023 & - & 7.32 & 6.64 & 5.55 & 0.05 & 83.3 & 11.51 & HiSeq4000 \\
\hline TDr_070 & TDr09/00028 & - & 9.46 & 8.59 & 6.94 & 0.08 & 83.9 & 14.26 & HiSeq4000 \\
\hline TDr_071 & TDro9/00056 & - & 6.89 & 5.97 & 5.12 & 0.07 & 84.2 & 10.49 & HiSeq4000 \\
\hline TDr_072 & TDrog/00070 & - & 8.13 & 7.31 & 6.06 & 0.05 & 84.1 & 12.44 & HiSeq4000 \\
\hline TDr_073 & TDr09/00091 & - & 7.81 & 7.01 & 5.93 & 0.05 & 83.7 & 12.23 & HiSeq4000 \\
\hline TDr_074 & TDro9/00104 & - & 8.88 & 8.12 & 6.80 & 0.05 & 85.6 & 13.71 & HiSeq4000 \\
\hline TDr_075 & TDr09/00108 & - & 8.73 & 7.55 & 6.06 & 0.06 & 83.7 & 12.50 & HiSeq4000 \\
\hline TDr_076 & TDr09/00114 & - & 7.66 & 6.38 & 5.28 & 0.04 & 82.9 & 11.01 & HiSeq4000 \\
\hline TDr_077 & TDrog/00125 & - & 8.29 & 7.12 & 5.83 & 0.05 & 82.8 & 12.16 & HiSeq 4000 \\
\hline TDr_078 & TDrog/00134 & - & 5.53 & 4.51 & 3.74 & 0.03 & 79.3 & 8.14 & HiSeq 4000 \\
\hline TDr_079 & TDrog/00248 & - & 9.28 & 8.19 & 6.26 & 0.04 & 82.5 & 13.09 & HiSeq4000 \\
\hline TDr_080 & TDr09/00350 & - & 8.54 & 7.59 & 6.36 & 0.03 & 83.2 & 13.18 & HiSeq4000 \\
\hline TDr_081 & TDr99/02789 & - & 5.88 & 4.71 & 3.79 & 0.02 & 78.9 & 8.29 & HiSeq 4000 \\
\hline TDr_082 & TDr11/00263.1 & - & 5.02 & 4.25 & 3.73 & 0.04 & 82.3 & 7.81 & HiSeq 4000 \\
\hline TDr_083 & TDr08/00161 & - & 7.23 & 6.40 & 5.24 & 0.06 & 82.7 & 10.93 & HiSeq 4000 \\
\hline TDr_084 & TDr11/00799 & - & 13.32 & 11.78 & 9.92 & 0.07 & 88.2 & 19.40 & HiSeq4000 \\
\hline TDr_085 & TDr11/01041 & - & 8.72 & 7.87 & 6.55 & 0.06 & 86.2 & 13.12 & HiSeq 4000 \\
\hline TDr_086 & TDr12/00474 & - & 8.47 & 7.56 & 5.81 & 0.06 & 83.1 & 12.07 & HiSeq4000 \\
\hline TDr_087 & TDr08/00146 & - & 8.96 & 7.98 & 6.64 & 0.04 & 86.6 & 13.23 & HiSeq4000 \\
\hline TDr_088 & TDrAlumaco & - & 10.90 & 9.16 & 7.27 & 0.08 & 82.2 & 15.26 & HiSeq4000 \\
\hline TDr_089 & TDrHembakoase & - & 6.40 & 5.79 & 5.03 & 0.04 & 84.8 & 10.23 & HiSeq 4000 \\
\hline TDr_090 & TDr89/02665 & - & 11.01 & 9.62 & 8.24 & 0.08 & 86.3 & 16.48 & HiSeq4000 \\
\hline TDr_091 & TDr05/00046 & - & 8.32 & 7.32 & 5.35 & 0.06 & 80.9 & 11.41 & HiSeq 4000 \\
\hline TDr_092 & TDr05/00432 & - & 8.65 & 7.33 & 6.39 & 0.08 & 83.8 & 13.16 & HiSeq 4000 \\
\hline TDr_093 & TDr05/00389 & - & 5.53 & 4.60 & 3.74 & 0.03 & 79.4 & 8.14 & HiSeq 4000 \\
\hline TDr_094 & TDr08/00023 & - & 7.24 & 6.10 & 5.23 & 0.05 & 82.7 & 10.93 & HiSeq 4000 \\
\hline TDr_095 & TDr08/00115 & - & 7.82 & 6.64 & 5.84 & 0.08 & 85.6 & 11.78 & HiSeq4000 \\
\hline TDr_096 & TDr08/00197 & - & 9.05 & 7.81 & 6.66 & 0.04 & 85.4 & 13.45 & HiSeq 4000 \\
\hline TDr_097 & TDr08/00974 & - & 6.68 & 5.61 & 4.93 & 0.04 & 85.2 & 9.99 & HiSeq 4000 \\
\hline TDr_098 & TDr08/00896 & - & 8.32 & 7.54 & 6.41 & 0.04 & 85.1 & 12.99 & HiSeq 4000 \\
\hline TDr_099 & TDr08/00841 & - & 11.21 & 9.83 & 7.43 & 0.09 & 85.0 & 15.09 & HiSeq4000 \\
\hline TDr_100 & TDro836 & - & 7.49 & 6.51 & 4.97 & 0.06 & 79.3 & 10.82 & HiSeq 4000 \\
\hline TDr_101 & TDr1686 & - & 10.98 & 9.61 & 8.03 & 0.18 & 86.9 & 15.96 & HiSeq4000 \\
\hline TDr_102 & TDr3010 & - & 12.57 & 11.02 & 9.10 & 0.20 & 85.5 & 18.36 & HiSeq 4000 \\
\hline TDr_103 & TDr3357 & - & 11.48 & 10.24 & 8.63 & 0.13 & 85.5 & 17.41 & HiSeq4000 \\
\hline TDr_104 & TDr3408 & - & 11.17 & 9.98 & 8.39 & 0.13 & 86.1 & 16.81 & HiSeq 4000 \\
\hline TDr_105 & TDr3430 & - & 9.58 & 8.42 & 7.19 & 0.14 & 86.3 & 14.38 & HiSeq 4000 \\
\hline TDr_106 & TDr3519 & - & 9.88 & 8.82 & 6.71 & 0.29 & 85.9 & 13.48 & HiSeq4000 \\
\hline TDr_107 & TDr3567 & - & 10.00 & 8.74 & 7.24 & 0.21 & 85.4 & 14.63 & HiSeq 4000 \\
\hline TDr_108 & TDr3569 & - & 10.02 & 8.88 & 7.43 & 0.15 & 86.6 & 14.81 & HiSeq 4000 \\
\hline TDr_109 & TDr3579 & - & 8.82 & 7.71 & 5.91 & 0.30 & 85.8 & 11.88 & HiSeq4000 \\
\hline TDr_110 & TDr3592 & - & 8.67 & 7.71 & 5.90 & 0.25 & 85.7 & 11.89 & HiSeq 4000 \\
\hline TDr_111 & TDr3610 & - & 10.20 & 9.02 & 7.46 & 0.17 & 86.6 & 14.86 & HiSeq 4000 \\
\hline TDr_112 & TDr3663 & - & 10.92 & 9.65 & 8.10 & 0.15 & 86.3 & 16.21 & HiSeq4000 \\
\hline TDr_113 & TDr3814 & - & 11.07 & 9.68 & 8.13 & 0.13 & 86.7 & 16.19 & HiSeq 4000 \\
\hline TDr_114 & TDr3881 & - & 11.82 & 10.57 & 9.07 & 0.14 & 86.6 & 18.07 & HiSeq 4000 \\
\hline
\end{tabular}




\begin{tabular}{|c|c|}
\hline TDr_115 & TDr4028 \\
\hline TDr_116 & TDr08/00641 \\
\hline TDr_117 & TDr08/00756 \\
\hline TDr_118 & TDrog/00131 \\
\hline TDr_119 & TDr1569 \\
\hline TDr_120 & TDr2931 \\
\hline TDr_121 & TDr2331.1 \\
\hline TDr_122 & TDr1958 \\
\hline TDr_123 & TDr1905 \\
\hline TDr_124 & TDr1928 \\
\hline TDr_125 & TDr3322 \\
\hline TDr_126 & TDr2048 \\
\hline TDr_127 & TDr2126 \\
\hline TDr_128 & TDr2249 \\
\hline TDr_129 & TDr2297 \\
\hline TDr_130 & TDr2342 \\
\hline TDr_131 & TDr2355 \\
\hline TDr_132 & TDr2564 \\
\hline TDr_133 & TDr2698 \\
\hline TDr_134 & TDr2974 \\
\hline TDr_135 & TDr2975 \\
\hline TDr_136 & TDr3507 \\
\hline TDr_137 & TDr3006 \\
\hline TDr_138 & TDr08/00091 \\
\hline TDr_139 & TDr08/01464 \\
\hline TDr_140 & TDr08/00989 \\
\hline TDr_141 & TDr09/00050 \\
\hline TDr_142 & TDr09/00055 \\
\hline TDr_144 & TDro9/00061 \\
\hline TDr_145 & TDr09/00123 \\
\hline TDr_146 & TDrog/00124 \\
\hline TDr_147 & TDro9/00220 \\
\hline TDr_148 & TDr09/00280.1 \\
\hline TDr_149 & TDro9/00324 \\
\hline TDr_150 & TDr08/01046 \\
\hline TDr_151 & TDrAme \\
\hline TDr_152 & TDrUfenyi \\
\hline TDr_153 & TDr2365 \\
\hline TDr_154 & TDr1956 \\
\hline TDr_155 & TDr2859 \\
\hline TDr_156 & TDr07/000732 \\
\hline TDr_157 & TDro8/00764 \\
\hline TDr_158 & TDro9/00155 \\
\hline TDr_159 & TDr96/01724 \\
\hline TDr_160 & TDr08/01287 \\
\hline TDr_161 & TDr08/01090 \\
\hline TDr_162 & TDr2366 \\
\hline TDr_163 & TDr2467 \\
\hline TDr_164 & TDr3003 \\
\hline TDr_165 & TDr3294 \\
\hline TDr_166 & TDr3338 \\
\hline
\end{tabular}

\begin{tabular}{rr|rlll}
11.29 & 9.98 & 8.31 & 0.15 & 86.9 & 16.51 \\
11.86 & 10.49 & 8.78 & 0.18 & 85.5 & 17.72 \\
9.84 & 8.60 & 7.29 & 0.18 & 85.4 & 14.73 \\
9.12 & 7.95 & 6.77 & 0.12 & 84.9 & 13.76 \\
8.97 & 7.66 & 6.44 & 0.10 & 85.2 & 13.05 \\
9.01 & 7.86 & 6.83 & 0.10 & 85.1 & 13.84 \\
10.29 & 9.05 & 7.64 & 0.11 & 84.7 & 15.57 \\
8.66 & 7.55 & 5.85 & 0.22 & 85.6 & 11.80 \\
11.62 & 10.34 & 8.58 & 0.18 & 85.2 & 17.37 \\
10.93 & 9.73 & 8.29 & 0.11 & 86.1 & 16.61 \\
9.48 & 8.20 & 6.69 & 0.17 & 86.0 & 13.43 \\
9.82 & 8.67 & 7.23 & 0.15 & 85.5 & 14.59 \\
10.08 & 8.85 & 7.33 & 0.14 & 85.8 & 14.75 \\
9.38 & 8.29 & 6.23 & 0.23 & 85.5 & 12.59 \\
9.97 & 8.72 & 6.53 & 0.30 & 85.7 & 13.16 \\
11.08 & 9.61 & 7.73 & 0.16 & 86.2 & 15.48 \\
10.27 & 9.06 & 6.82 & 0.29 & 85.8 & 13.72 \\
8.75 & 7.63 & 6.49 & 0.13 & 85.7 & 13.07 \\
10.55 & 9.12 & 7.30 & 0.16 & 85.7 & 14.72 \\
12.48 & 11.01 & 8.18 & 0.32 & 86.5 & 16.33 \\
9.23 & 8.17 & 6.20 & 0.24 & 85.6 & 12.50 \\
10.29 & 9.05 & 7.67 & 0.11 & 85.3 & 15.52 \\
10.03 & 8.85 & 7.68 & 0.12 & 85.1 & 15.59 \\
7.14 & 6.30 & 5.43 & 0.08 & 83.8 & 11.18 \\
7.29 & 6.42 & 5.59 & 0.06 & 84.4 & 11.44 \\
6.96 & 6.07 & 5.18 & 0.09 & 83.3 & 10.72 \\
7.43 & 6.50 & 5.51 & 0.07 & 84.3 & 11.29 \\
9.41 & 8.24 & 6.96 & 0.12 & 84.1 & 14.28 \\
9.15 & 7.96 & 6.85 & 0.09 & 85.6 & 13.80 \\
8.34 & 7.28 & 6.13 & 0.10 & 83.9 & 12.62 \\
8.76 & 7.72 & 6.59 & 0.12 & 85.0 & 13.39 \\
13.21 & 11.50 & 9.30 & 0.15 & 85.8 & 18.70 \\
8.31 & 7.30 & 6.22 & 0.08 & 84.4 & 12.73 \\
7.26 & 6.27 & 5.39 & 0.09 & 83.0 & 11.20 \\
12.03 & 10.56 & 8.82 & 0.17 & 86.2 & 17.65 \\
14.49 & 12.87 & 10.65 & 0.26 & 85.9 & 21.39 \\
12.72 & 11.18 & 9.11 & 0.33 & 86.6 & 18.15 \\
12.77 & 11.26 & 9.52 & 0.21 & 85.4 & 19.25 \\
10.78 & 9.74 & 8.42 & 0.14 & 86.0 & 16.91 \\
11.25 & 10.13 & 7.87 & 0.26 & 86.3 & 15.73 \\
9.98 & 8.91 & 7.72 & 0.06 & 85.1 & 15.66 \\
10.88 & 9.73 & 8.33 & 0.09 & 85.7 & 16.77 \\
9.60 & 8.63 & 7.45 & 0.08 & 86.5 & 14.86 \\
8.68 & 7.74 & 6.72 & 0.08 & 84.9 & 13.65 \\
8.54 & 7.67 & 6.68 & 0.06 & 85.6 & 13.47 \\
9.38 & 8.41 & 7.29 & 0.05 & 84.9 & 14.82 \\
9.63 & 8.50 & 7.08 & 0.06 & 83.8 & 14.58 \\
9.49 & 8.55 & 7.44 & 0.04 & 85.2 & 15.08 \\
9.10 & 8.14 & 7.08 & 0.09 & 85.2 & 14.35 \\
8.55 & 7.66 & 6.69 & 0.06 & 85.9 & 13.44 \\
11.19 & 10.03 & 8.66 & 0.11 & 85.2 & 17.54
\end{tabular}

HiSeq4000
HiSeq4000
HiSeq4000
HiSeq4000
HiSeq4000
HiSeq4000
HiSeq4000
HiSeq4000
HiSeq4000
HiSeq4000
HiSeq4000
HiSeq4000
HiSeq4000
HiSeq4000
HiSeq4000
HiSeq4000
HiSeq4000
HiSeq4000
HiSeq4000
HiSeq4000
HiSeq4000
HiSeq4000
HiSeq4000
HiSeq4000
HiSeq4000
HiSeq4000
HiSeq4000
HiSeq4000
HiSeq4000
HiSeq4000
HiSeq4000
HiSeq4000
HiSeq4000
HiSeq4000
HiSeq4000
HiSeq4000
HiSeq4000
HiSeq4000
HiSeq4000
HiSeq4000
HiSeq4000
HiSeq4000
HiSeq4000
HiSeq4000
HiSeq4000
HiSeq4000
HiSeq4000
HiSeq4000
HiSeq4000
HiSeq4000
HSSeq4000

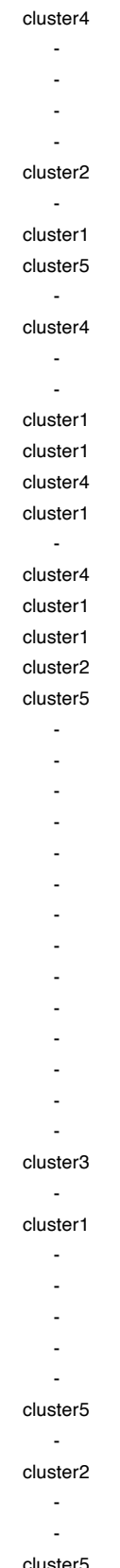




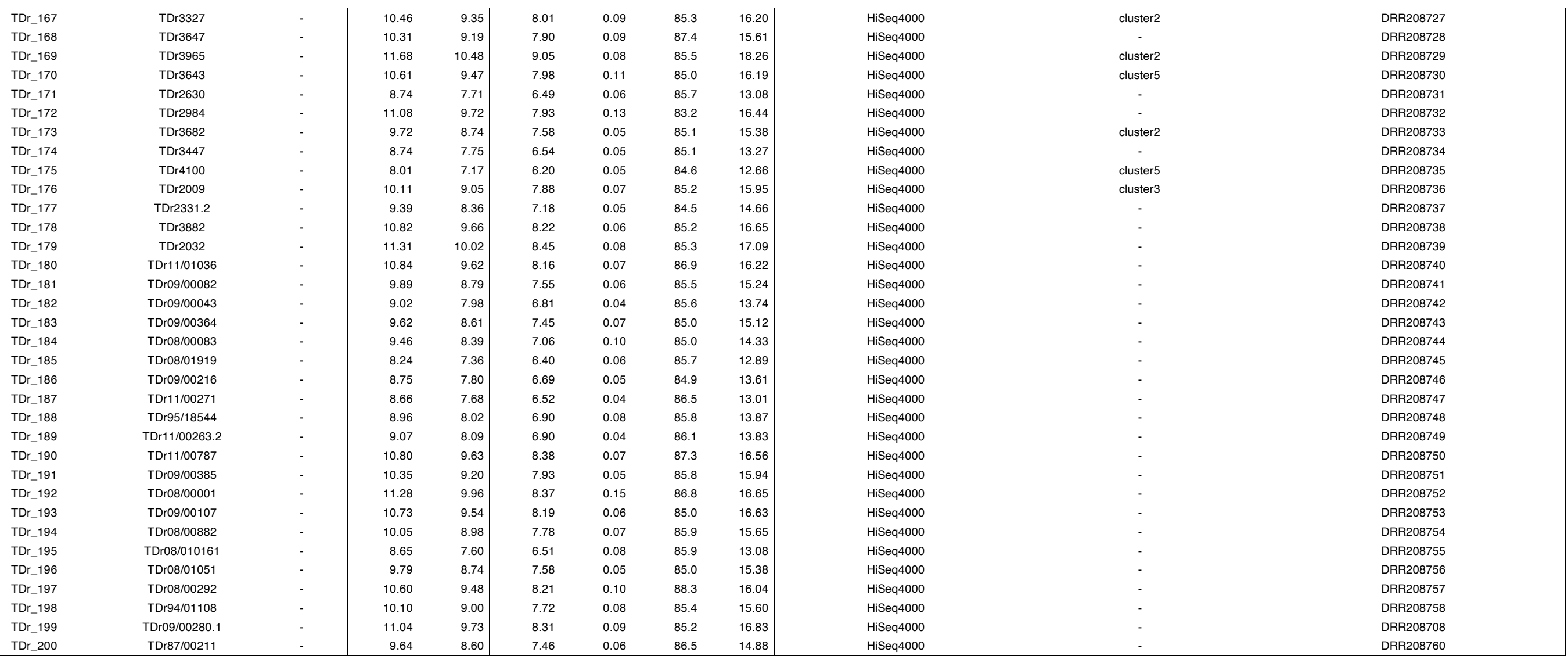


Table SM7. Summary of sequence alignment of mapping population

\begin{tabular}{|c|c|c|c|c|c|c|c|c|c|c|}
\hline \multicolumn{2}{|c|}{ Sample } & \multicolumn{2}{|c|}{ Fastq size } & \multicolumn{4}{|c|}{ Aligned bam information } & \multirow[b]{2}{*}{ Sequence platform } & \multirow[b]{2}{*}{ Comment } & \multirow[b]{2}{*}{ Accession No. } \\
\hline Name & IITA name & $\begin{array}{l}\text { Original } \\
\text { (Gbp) }\end{array}$ & $\begin{array}{l}\text { Filtered } \\
\text { (Gbp) }\end{array}$ & $\begin{array}{l}\text { Aligned } \\
\text { (Gbp) }\end{array}$ & $\begin{array}{c}\text { Unmapped } \\
\text { (Gbp) }\end{array}$ & $\begin{array}{c}\text { Coverage } \\
(\%)\end{array}$ & Depth & & & \\
\hline TDr04_219 & TDr04_219 & 38.26 & 33.10 & 17.15 & 0.32 & 82.8 & 35.73 & MiSeq, HiSeq4000, GAllx & MP2 family Mono parent & DRR208404,DRR208405,DRR063085 \\
\hline TDr97_777 & TDr97_777 & 25.47 & 22.71 & 11.20 & 0.29 & 79.4 & 24.35 & MiSeq,HiSeq4000,NextSeq500,GAllx & MP2 family Male parent & DRR063127,DRR208406,DRR045130-7,DRR063111 \\
\hline MP2_001 & MP2_001 & 8.20 & 7.14 & 4.20 & 1.00 & 76.9 & 9.43 & HiSeq4000 & - & DRR208407 \\
\hline MP2_002 & MP2_002 & 6.42 & 5.61 & 3.45 & 0.64 & 73.2 & 8.13 & HiSeq4000 & - & DRR208408 \\
\hline MP2_003 & MP2_003 & 5.95 & 5.11 & 2.92 & 0.87 & 71.6 & 7.03 & HiSeq4000 & - & DRR208409 \\
\hline MP2_004 & MP2_004 & 7.13 & 6.24 & 3.90 & 0.70 & 74.8 & 8.99 & HiSeq4000 & - & DRR208410 \\
\hline MP2_005 & MP2_005 & 9.75 & 8.49 & 4.59 & 1.56 & 75.2 & 10.53 & HiSeq4000 & - & DRR208411 \\
\hline MP2_006 & MP2_006 & 7.90 & 7.01 & 4.39 & 0.76 & 77.2 & 9.80 & HiSeq4000 & - & DRR208412 \\
\hline MP2_007 & MP2_007 & 7.50 & 6.57 & 4.11 & 0.75 & 75.8 & 9.35 & HiSeq4000 & - & DRR208413 \\
\hline MP2_008 & MP2_008 & 7.52 & 6.60 & 3.93 & 0.81 & 74.3 & 9.13 & HiSeq4000 & - & DRR208414 \\
\hline MP2_009 & MP2_009 & 7.36 & 6.48 & 4.12 & 0.62 & 76.3 & 9.33 & HiSeq4000 & - & DRR208415 \\
\hline MP2_010 & MP2_010 & 6.49 & 5.72 & 3.66 & 0.55 & 75.2 & 8.39 & HiSeq4000 & - & DRR208416 \\
\hline MP2_011 & MP2_011 & 5.98 & 5.28 & 3.41 & 0.49 & 77.1 & 7.63 & HiSeq4000 & - & DRR208417 \\
\hline MP2_012 & MP2_012 & 8.25 & 7.31 & 4.69 & 0.77 & 76.9 & 10.53 & HiSeq4000 & - & DRR208418 \\
\hline MP2_013 & MP2_013 & 9.33 & 8.05 & 4.81 & 1.00 & 76.2 & 10.89 & HiSeq4000 & - & DRR208419 \\
\hline MP2_014 & MP2_014 & 9.84 & 8.65 & 5.56 & 0.81 & 78.0 & 12.32 & HiSeq4000 & - & DRR208420 \\
\hline MP2_015 & MP2_015 & 11.21 & 9.80 & 6.29 & 0.93 & 78.5 & 13.82 & HiSeq4000 & - & DRR208421 \\
\hline MP2_016 & MP2_016 & 12.97 & 11.36 & 6.86 & 1.18 & 78.1 & 15.15 & HiSeq4000 & - & DRR208422 \\
\hline MP2_017 & MP2_017 & 3.89 & 2.96 & 1.48 & 0.36 & 67.0 & 3.83 & HiSeq4000 & - & DRR208423 \\
\hline MP2_018 & MP2_018 & 12.70 & 11.17 & 7.04 & 1.10 & 78.3 & 15.53 & HiSeq4000 & - & DRR208424 \\
\hline MP2_019 & MP2_019 & 5.00 & 4.31 & 2.32 & 0.41 & 74.2 & 5.38 & HiSeq4000 & - & DRR208425 \\
\hline MP2_020 & MP2_020 & 10.13 & 9.04 & 6.04 & 0.78 & 78.1 & 13.34 & HiSeq4000 & - & DRR208426 \\
\hline MP2_023 & MP2_023 & 4.98 & 3.90 & 2.10 & 0.35 & 71.4 & 5.08 & HiSeq4000 & - & DRR208427 \\
\hline MP2_024 & MP2_024 & 10.08 & 8.74 & 5.10 & 1.27 & 75.4 & 11.68 & HiSeq4000 & - & DRR208428 \\
\hline MP2_025 & MP2_025 & 4.80 & 3.53 & 1.91 & 0.38 & 70.2 & 4.70 & HiSeq4000 & - & DRR208429 \\
\hline MP2_026 & MP2_026 & 8.36 & 7.38 & 4.88 & 0.66 & 77.5 & 10.86 & HiSeq4000 & - & DRR208430 \\
\hline MP2_027 & MP2_027 & 5.35 & 3.86 & 2.05 & 0.37 & 71.6 & 4.93 & HiSeq4000 & - & DRR208431 \\
\hline MP2_028 & MP2_028 & 8.11 & 7.08 & 4.45 & 0.72 & 76.4 & 10.05 & HiSeq4000 & - & DRR208432 \\
\hline MP2_029 & MP2_029 & 9.89 & 8.61 & 5.03 & 1.08 & 75.4 & 11.52 & HiSeq4000 & - & DRR208433 \\
\hline MP2_031 & MP2_031 & 10.33 & 9.08 & 6.04 & 0.79 & 78.5 & 13.30 & HiSeq4000 & - & DRR208434 \\
\hline MP2_032 & MP2_032 & 16.56 & 12.57 & 6.45 & 1.21 & 78.9 & 14.12 & HiSeq4000 & - & DRR208435 \\
\hline MP2_033 & MP2_033 & 7.32 & 6.41 & 4.19 & 0.62 & 77.5 & 9.34 & HiSeq4000 & - & DRR208436 \\
\hline MP2_034 & MP2_034 & 8.05 & 6.99 & 4.40 & 0.79 & 75.0 & 10.12 & HiSeq4000 & - & DRR208437 \\
\hline MP2_035 & MP2_035 & 9.06 & 7.95 & 4.96 & 0.83 & 77.3 & 11.07 & HiSeq4000 & - & DRR208438 \\
\hline MP2_037 & MP2_037 & 9.70 & 8.41 & 5.16 & 0.99 & 77.3 & 11.53 & HiSeq4000 & - & DRR208439 \\
\hline MP2_039 & MP2_039 & 7.54 & 6.58 & 4.00 & 0.82 & 75.4 & 9.17 & HiSeq4000 & - & DRR208440 \\
\hline MP2_043 & MP2_043 & 9.15 & 7.93 & 4.24 & 0.71 & 77.3 & 9.46 & HiSeq4000 & - & DRR208441 \\
\hline MP2_044 & MP2_044 & 9.75 & 8.60 & 5.28 & 0.95 & 76.9 & 11.85 & HiSeq4000 & - & DRR208442 \\
\hline MP2_047 & MP2_047 & 8.95 & 7.64 & 4.04 & 0.76 & 77.1 & 9.03 & HiSeq4000 & - & DRR208443 \\
\hline MP2_048 & MP2_048 & 8.27 & 7.24 & 3.94 & 0.69 & 77.4 & 8.80 & HiSeq4000 & - & DRR208444 \\
\hline MP2_050 & MP2_050 & 11.17 & 9.77 & 5.67 & 1.35 & 76.2 & 12.85 & HiSeq4000 & - & DRR208445 \\
\hline MP2_052 & MP2_052 & 9.98 & 8.75 & 5.18 & 1.13 & 75.1 & 11.90 & HiSeq4000 & - & DRR208446 \\
\hline MP2_053 & MP2_053 & 11.85 & 9.88 & 4.74 & 2.21 & 72.0 & 11.37 & HiSeq4000 & - & DRR208447 \\
\hline MP2_054 & MP2_054 & 10.38 & 6.95 & 3.67 & 0.70 & 77.1 & 8.21 & HiSeq4000 & - & DRR208448 \\
\hline MP2_055 & MP2_055 & 12.74 & 10.66 & 5.55 & 1.85 & 74.8 & 12.81 & HiSeq4000 & - & DRR208449 \\
\hline
\end{tabular}




\begin{tabular}{|c|c|c|c|c|c|c|c|c|}
\hline MP2_057 & MP2_057 & 8.68 & 7.41 & 4.06 & 1.24 & 72.2 & 9.72 & HiSeq4000 \\
\hline MP2_058 & MP2_058 & 11.14 & 9.54 & 6.10 & 0.89 & 78.2 & 13.47 & HiSeq4000 \\
\hline MP2_060 & MP2_060 & 8.31 & 7.05 & 3.51 & 0.79 & 76.0 & 7.97 & HiSeq4000 \\
\hline MP2_061 & MP2_061 & 12.07 & 10.38 & 6.88 & 0.95 & 79.0 & 15.04 & HiSeq4000 \\
\hline MP2_063 & MP2_063 & 7.03 & 5.43 & 2.96 & 0.51 & 76.3 & 6.71 & HiSeq4000 \\
\hline MP2_064 & MP2_064 & 11.23 & 9.50 & 5.46 & 1.28 & 76.0 & 12.39 & HiSeq4000 \\
\hline MP2_113 & MP2_113 & 6.79 & 5.71 & 3.29 & 0.79 & 75.0 & 7.57 & HiSeq4000 \\
\hline MP2_114 & MP2_114 & 7.80 & 6.62 & 3.60 & 0.94 & 70.9 & 8.75 & HiSeq4000 \\
\hline MP2_116 & MP2_116 & 7.17 & 6.14 & 3.78 & 0.66 & 75.5 & 8.64 & HiSeq4000 \\
\hline MP2_117 & MP2_117 & 6.52 & 5.53 & 3.38 & 0.55 & 75.9 & 7.69 & HiSeq4000 \\
\hline MP2_121 & MP2_121 & 11.64 & 10.04 & 5.72 & 1.45 & 76.1 & 12.96 & HiSeq4000 \\
\hline MP2_122 & MP2_122 & 9.07 & 7.65 & 4.33 & 1.15 & 75.5 & 9.89 & HiSeq4000 \\
\hline MP2_125 & MP2_125 & 9.25 & 8.04 & 4.87 & 0.86 & 77.7 & 10.82 & HiSeq4000 \\
\hline MP2_126 & MP2_126 & 8.65 & 7.46 & 4.36 & 1.00 & 76.1 & 9.89 & HiSeq4000 \\
\hline MP2_127 & MP2_127 & 11.45 & 9.94 & 6.22 & 0.99 & 78.0 & 13.76 & HiSeq4000 \\
\hline MP2_128 & MP2_128 & 10.17 & 8.91 & 5.41 & 1.01 & 77.1 & 12.11 & HiSeq4000 \\
\hline MP2_129 & MP2_129 & 11.75 & 10.05 & 5.97 & 1.32 & 77.4 & 13.30 & HiSeq4000 \\
\hline MP2_130 & MP2_130 & 9.04 & 7.78 & 4.94 & 0.75 & 76.8 & 11.10 & HiSeq4000 \\
\hline MP2_131 & MP2_131 & 10.02 & 8.69 & 5.59 & 0.85 & 78.2 & 12.34 & HiSeq4000 \\
\hline MP2_132 & MP2_132 & 9.93 & 8.56 & 5.23 & 0.99 & 77.2 & 11.69 & HiSeq4000 \\
\hline MP2_133 & MP2_133 & 7.97 & 6.87 & 4.29 & 0.71 & 77.0 & 9.63 & HiSeq4000 \\
\hline MP2_136 & MP2_136 & 9.56 & 8.20 & 4.48 & 1.48 & 76.2 & 10.14 & HiSeq4000 \\
\hline MP2_137 & MP2_137 & 10.99 & 9.51 & 5.70 & 1.15 & 76.5 & 12.86 & HiSeq4000 \\
\hline MP2_138 & MP2_138 & 8.51 & 7.42 & 4.61 & 0.76 & 77.3 & 10.28 & HiSeq4000 \\
\hline MP2_139 & MP2_139 & 9.41 & 8.27 & 5.12 & 0.83 & 75.9 & 11.65 & HiSeq4000 \\
\hline MP2_140 & MP2_140 & 8.91 & 7.74 & 4.74 & 0.90 & 76.9 & 10.65 & HiSeq4000 \\
\hline MP2_141 & MP2_141 & 9.22 & 7.61 & 4.05 & 1.22 & 72.2 & 9.69 & HiSeq4000 \\
\hline MP2_142 & MP2_142 & 10.72 & 9.12 & 4.11 & 2.49 & 73.3 & 9.67 & HiSeq4000 \\
\hline MP2_143 & MP2_143 & 7.99 & 6.94 & 4.03 & 0.91 & 75.3 & 9.24 & HiSeq4000 \\
\hline MP2_144 & MP2_144 & 9.30 & 8.14 & 5.31 & 0.79 & 77.5 & 11.83 & HiSeq4000 \\
\hline MP2_145 & MP2_145 & 10.35 & 8.99 & 5.13 & 1.17 & 76.5 & 11.56 & HiSeq4000 \\
\hline MP2_146 & MP2_146 & 10.87 & 9.44 & 5.39 & 1.41 & 77.1 & 12.07 & HiSeq4000 \\
\hline MP2_147 & MP2_147 & 9.96 & 8.80 & 5.79 & 0.76 & 78.4 & 12.75 & HiSeq4000 \\
\hline MP2_149 & MP2_149 & 9.80 & 8.64 & 5.74 & 0.78 & 78.0 & 12.71 & HiSeq4000 \\
\hline MP2_150 & MP2_150 & 7.47 & 6.31 & 3.17 & 1.22 & 71.5 & 7.65 & HiSeq4000 \\
\hline MP2_151 & MP2_151 & 8.96 & 7.85 & 4.80 & 0.90 & 78.0 & 10.63 & HiSeq4000 \\
\hline MP2_152 & MP2_152 & 12.30 & 10.66 & 6.41 & 1.29 & 78.8 & 14.02 & HiSeq4000 \\
\hline MP2_154 & MP2_154 & 9.78 & 8.41 & 4.56 & 1.42 & 75.8 & 10.38 & HiSeq4000 \\
\hline MP2_155 & MP2_155 & 10.40 & 9.01 & 5.31 & 1.23 & 77.5 & 11.82 & HiSeq4000 \\
\hline MP2_156 & MP2_156 & 8.67 & 7.49 & 4.32 & 1.00 & 76.2 & 9.79 & HiSeq4000 \\
\hline MP2_157 & MP2_157 & 7.64 & 6.64 & 4.00 & 0.84 & 76.0 & 9.08 & HiSeq4000 \\
\hline MP2_158 & MP2_158 & 8.84 & 7.67 & 4.85 & 0.79 & 77.8 & 10.77 & HiSeq4000 \\
\hline MP2_159 & MP2_159 & 9.82 & 8.47 & 4.97 & 1.16 & 77.2 & 11.10 & HiSeq4000 \\
\hline MP2_160 & MP2_160 & 8.43 & 7.33 & 4.57 & 0.73 & 77.2 & 10.23 & HiSeq4000 \\
\hline MP2_161 & MP2_161 & 8.93 & 7.71 & 4.46 & 1.10 & 77.1 & 9.99 & HiSeq4000 \\
\hline MP2_162 & MP2_162 & 12.11 & 10.46 & 5.71 & 1.62 & 77.4 & 12.73 & HiSeq4000 \\
\hline MP2_166 & MP2_166 & 12.03 & 10.49 & 6.27 & 1.21 & 76.7 & 14.09 & HiSeq4000 \\
\hline MP2_167 & MP2_167 & 9.67 & 8.39 & 4.63 & 1.31 & 74.7 & 10.70 & HiSeq4000 \\
\hline MP2_168 & MP2_168 & 15.43 & 13.47 & 8.68 & 1.28 & 79.0 & 18.96 & HiSeq4000 \\
\hline
\end{tabular}




\begin{tabular}{|c|c|c|c|c|c|c|c|c|}
\hline MP2_169 & MP2_169 & 12.87 & 11.15 & 6.58 & 1.40 & 77.7 & 14.62 & HiSeq4000 \\
\hline MP2_170 & MP2_170 & 13.20 & 11.31 & 6.24 & 1.83 & 77.3 & 13.94 & HiSeq4000 \\
\hline MP2_172 & MP2_172 & 11.50 & 9.60 & 5.68 & 1.08 & 75.6 & 12.97 & HiSeq4000 \\
\hline MP2_173 & MP2_173 & 10.20 & 8.86 & 4.90 & 1.31 & 74.9 & 11.28 & HiSeq4000 \\
\hline MP2_174 & MP2_174 & 10.70 & 9.28 & 5.37 & 1.26 & 77.7 & 11.95 & HiSeq4000 \\
\hline MP2_175 & MP2_175 & 13.09 & 11.51 & 7.00 & 1.21 & 77.4 & 15.60 & HiSeq4000 \\
\hline MP2_177 & MP2_177 & 6.33 & 5.38 & 2.88 & 1.00 & 71.7 & 6.93 & HiSeq4000 \\
\hline MP2_178 & MP2_178 & 5.89 & 5.10 & 3.00 & 0.66 & 73.2 & 7.07 & HiSeq4000 \\
\hline MP2_179 & MP2_179 & 4.55 & 3.89 & 2.47 & 0.42 & 73.5 & 5.79 & HiSeq4000 \\
\hline MP2_180 & MP2_180 & 7.09 & 6.10 & 3.54 & 0.86 & 74.8 & 8.17 & HiSeq4000 \\
\hline MP2_181 & MP2_181 & 6.41 & 5.45 & 3.05 & 0.91 & 72.6 & 7.26 & HiSeq4000 \\
\hline MP2_182 & MP2_182 & 8.34 & 7.16 & 4.72 & 0.71 & 78.2 & 10.42 & HiSeq4000 \\
\hline MP2_183 & MP2_183 & 8.89 & 7.74 & 5.12 & 0.74 & 77.0 & 11.47 & HiSeq4000 \\
\hline MP2_185 & MP2_185 & 6.46 & 5.49 & 3.06 & 0.97 & 72.4 & 7.30 & HiSeq4000 \\
\hline MP2_186 & MP2_186 & 6.37 & 5.37 & 3.39 & 0.59 & 76.0 & 7.70 & HiSeq4000 \\
\hline MP2_187 & MP2_187 & 5.86 & 4.97 & 2.86 & 0.72 & 72.4 & 6.83 & HiSeq4000 \\
\hline MP2_188 & MP2_188 & 8.36 & 7.11 & 4.48 & 0.83 & 76.4 & 10.12 & HiSeq4000 \\
\hline MP2_189 & MP2_189 & 6.63 & 5.69 & 3.34 & 0.75 & 73.9 & 7.80 & HiSeq4000 \\
\hline MP2_190 & MP2_190 & 6.41 & 5.35 & 3.44 & 0.58 & 77.4 & 7.67 & HiSeq4000 \\
\hline MP2_191 & MP2_191 & 7.46 & 6.22 & 3.76 & 0.85 & 74.9 & 8.67 & HiSeq4000 \\
\hline MP2_192 & MP2_192 & 6.76 & 5.71 & 3.54 & 0.64 & 74.8 & 8.16 & HiSeq4000 \\
\hline MP2_193 & MP2_193 & 9.63 & 8.56 & 5.41 & 0.86 & 77.5 & 12.06 & HiSeq4000 \\
\hline MP2_196 & MP2_196 & 11.11 & 9.85 & 6.23 & 0.96 & 78.2 & 13.76 & HiSeq4000 \\
\hline MP2_197 & MP2_197 & 7.35 & 6.22 & 3.96 & 0.66 & 76.6 & 8.92 & HiSeq4000 \\
\hline MP2_198 & MP2_198 & 8.72 & 7.48 & 4.86 & 0.74 & 78.2 & 10.74 & HiSeq4000 \\
\hline MP2_199 & MP2_199 & 6.66 & 5.90 & 3.58 & 0.69 & 74.8 & 8.25 & HiSeq4000 \\
\hline MP2_200 & MP2_200 & 7.00 & 6.22 & 3.99 & 0.61 & 75.8 & 9.08 & HiSeq4000 \\
\hline MP2_201 & MP2_201 & 8.36 & 7.17 & 4.39 & 0.86 & 75.4 & 10.06 & HiSeq4000 \\
\hline MP2_202 & MP2_202 & 9.03 & 7.71 & 3.83 & 1.87 & 74.4 & 8.88 & HiSeq4000 \\
\hline MP2_203 & MP2_203 & 7.58 & 6.73 & 4.06 & 0.76 & 76.8 & 9.12 & HiSeq4000 \\
\hline MP2_204 & MP2_204 & 10.55 & 9.21 & 5.02 & 1.48 & 77.2 & 11.22 & HiSeq4000 \\
\hline MP2_205 & MP2_205 & 11.71 & 10.10 & 6.18 & 1.22 & 77.5 & 13.76 & HiSeq4000 \\
\hline MP2_206 & MP2_206 & 8.72 & 7.29 & 3.94 & 1.43 & 74.1 & 9.16 & HiSeq4000 \\
\hline MP2_208 & MP2_208 & 11.54 & 10.28 & 6.41 & 1.12 & 78.2 & 14.16 & HiSeq4000 \\
\hline MP2_211 & MP2_211 & 9.81 & 8.70 & 5.44 & 1.02 & 78.4 & 11.98 & HiSeq4000 \\
\hline MP2_213 & MP2_213 & 10.05 & 8.77 & 5.30 & 1.02 & 78.0 & 11.73 & HiSeq4000 \\
\hline MP2_214 & MP2_214 & 8.64 & 7.69 & 4.64 & 0.96 & 76.1 & 10.53 & HiSeq4000 \\
\hline MP2_215 & MP2_215 & 9.92 & 8.76 & 5.62 & 0.81 & 78.0 & 12.43 & HiSeq4000 \\
\hline MP2_216 & MP2_216 & 9.92 & 8.64 & 5.19 & 1.10 & 75.4 & 11.88 & HiSeq4000 \\
\hline MP2_218 & MP2_218 & 9.62 & 8.52 & 5.24 & 1.10 & 75.4 & 11.99 & HiSeq4000 \\
\hline MP2_219 & MP2_219 & 7.57 & 6.57 & 4.15 & 0.70 & 74.8 & 9.57 & HiSeq4000 \\
\hline MP2_220 & MP2_220 & 7.81 & 6.90 & 4.21 & 0.78 & 76.1 & 9.55 & HiSeq4000 \\
\hline MP2_221 & MP2_221 & 9.33 & 8.28 & 5.13 & 0.92 & 76.2 & 11.63 & HiSeq4000 \\
\hline MP2_222 & MP2_222 & 9.13 & 7.90 & 4.79 & 1.02 & 75.7 & 10.93 & HiSeq4000 \\
\hline MP2_224 & MP2_224 & 11.19 & 9.85 & 6.23 & 1.05 & 77.1 & 13.95 & HiSeq4000 \\
\hline MP2_225 & MP2_225 & 8.97 & 7.74 & 4.41 & 1.09 & 74.2 & 10.25 & HiSeq4000 \\
\hline MP2_227 & MP2_227 & 14.19 & 12.43 & 7.97 & 1.15 & 78.7 & 17.48 & HiSeq4000 \\
\hline MP2_228 & MP2_228 & 9.03 & 7.86 & 4.92 & 0.90 & 76.8 & 11.05 & HiSeq4000 \\
\hline MP2_229 & MP2_229 & 10.39 & 9.13 & 5.71 & 0.97 & 77.5 & 12.73 & HiSeq4000 \\
\hline
\end{tabular}




\begin{tabular}{|c|c|c|c|c|c|c|c|c|c|c|}
\hline MP2_231 & MP2_231 & 10.31 & 8.99 & 5.62 & 0.96 & 77.6 & 12.50 & HiSeq4000 & - & DRR208548 \\
\hline MP2_232 & MP2_232 & 11.06 & 9.64 & 6.00 & 1.04 & 77.1 & 13.41 & HiSeq4000 & - & DRR208549 \\
\hline MP2_233 & MP2_233 & 9.57 & 8.46 & 5.23 & 1.07 & 76.8 & 11.76 & HiSeq4000 & - & DRR208550 \\
\hline MP2_234 & MP2_234 & 6.96 & 6.02 & 3.42 & 0.89 & 73.4 & 8.05 & HiSeq4000 & - & DRR208551 \\
\hline MP2_235 & MP2_235 & 8.71 & 7.54 & 4.21 & 1.25 & 73.9 & 9.82 & HiSeq4000 & - & DRR208552 \\
\hline MP2_237 & MP2_237 & 6.46 & 5.55 & 3.27 & 0.80 & 74.2 & 7.61 & HiSeq4000 & - & DRR208554 \\
\hline MP2_239 & MP2_239 & 7.08 & 6.14 & 3.77 & 0.73 & 75.0 & 8.66 & HiSeq4000 & - & DRR208555 \\
\hline MP2_240 & MP2_240 & 6.92 & 6.00 & 3.70 & 0.78 & 74.4 & 8.59 & HiSeq4000 & - & DRR208556 \\
\hline MP2_241 & MP2_241 & 10.28 & 8.87 & 4.73 & 1.60 & 74.7 & 10.92 & HiSeq4000 & - & DRR208557 \\
\hline MP2_242 & MP2_242 & 8.82 & 7.65 & 4.62 & 0.85 & 75.3 & 10.58 & HiSeq4000 & - & DRR208558 \\
\hline MP2_246 & MP2_246 & 6.86 & 5.98 & 3.77 & 0.70 & 76.6 & 8.50 & HiSeq4000 & - & DRR208560 \\
\hline MP2_247 & MP2_247 & 6.97 & 6.01 & 3.70 & 0.65 & 74.3 & 8.61 & HiSeq4000 & - & DRR208561 \\
\hline MP2_248 & MP2_248 & 6.45 & 5.60 & 3.62 & 0.57 & 76.7 & 8.14 & HiSeq4000 & - & DRR208562 \\
\hline
\end{tabular}


Table SM11. All sequence information of ourgroups.

\begin{tabular}{|c|c|c|c|c|c|c|c|c|c|}
\hline \multicolumn{2}{|c|}{ Sample } & \multicolumn{2}{|c|}{ Fastq size } & \multicolumn{4}{|c|}{ Aligned bam information } & \multirow[b]{2}{*}{ Comment } & \multirow[b]{2}{*}{ Accession No. } \\
\hline Name & Name in Scarcelli et al. 2019 & $\begin{array}{l}\text { Original } \\
\text { (Gbp) }\end{array}$ & $\begin{array}{c}\text { Filtered } \\
\text { (Gbp) }\end{array}$ & $\begin{array}{l}\text { Aligned } \\
\text { (Gbp) }\end{array}$ & $\begin{array}{c}\text { Unmapped } \\
\text { (Gbp) }\end{array}$ & $\begin{array}{c}\text { Coverage } \\
(\%)\end{array}$ & Depth & & \\
\hline alata1 & & 28.11 & 23.95 & 10.73 & 1.24 & 48.0 & 38.59 & D.alata & ERR1019033 \\
\hline alata2 & & 11.58 & 11.15 & 3.88 & 1.37 & 43.1 & 15.54 & D.alata & SRR7062294 \\
\hline ns004_A5689 & A5689 & 4.22 & 4.19 & 3.09 & 0.34 & 75.2 & 7.09 & D.abyssinica:Nigeria & SRR8451439 \\
\hline ns005_A5690 & A5690 & 5.79 & 5.72 & 4.06 & 0.37 & 68.5 & 10.24 & D.abyssinica:Nigeria & SRR8451438 \\
\hline ns006_A5691 & A5691 & 5.53 & 5.49 & 2.85 & 1.73 & 68.4 & 7.20 & D.abyssinica:Nigeria & SRR8451437 \\
\hline ns007_A5693 & A5693 & 5.93 & 5.89 & 4.54 & 0.15 & 78.3 & 10.01 & D.abyssinica:Nigeria & SRR8451434 \\
\hline ns008_A5694 & A5694 & 4.87 & 4.84 & 3.91 & 0.04 & 77.3 & 8.72 & D.abyssinica:Nigeria & SRR8451371 \\
\hline ns009_A5695 & A5695 & 4.55 & 4.52 & 3.35 & 0.42 & 78.4 & 7.37 & D.abyssinica:Nigeria & SRR8451459 \\
\hline ns010_A5696 & A5696 & 4.75 & 4.61 & 3.55 & 0.22 & 74.9 & 8.17 & D.abyssinica:Nigeria & SRR8451458 \\
\hline ns011_A5697 & A5697 & 5.70 & 5.66 & 4.41 & 0.15 & 80.2 & 9.48 & D.abyssinica:Nigeria & SRR8451382 \\
\hline ns012_A5699 & A5699 & 3.25 & 3.22 & 2.45 & 0.15 & 71.8 & 5.89 & D.abyssinica:Nigeria & SRR8451381 \\
\hline ns013_A5700 & A5700 & 4.79 & 4.76 & 3.59 & 0.32 & 77.0 & 8.05 & D.abyssinica:Nigeria & SRR8451384 \\
\hline ns014_A5701 & A5701 & 5.99 & 5.95 & 4.38 & 0.37 & 78.6 & 9.62 & D.abyssinica:Nigeria & SRR8451383 \\
\hline ns015_A5702 & A5702 & 3.96 & 3.93 & 2.95 & 0.29 & 74.9 & 6.79 & D.abyssinica:Nigeria & SRR8451378 \\
\hline ns016_A5703 & A5703 & 4.53 & 4.49 & 3.09 & 0.37 & 65.3 & 8.17 & D.abyssinica:Nigeria & SRR8451377 \\
\hline ns017_A5704 & A5704 & 4.95 & 4.91 & 2.85 & 1.17 & 69.6 & 7.08 & D.abyssinica:Nigeria & SRR8451380 \\
\hline ns018_A5705 & A5705 & 5.54 & 5.49 & 3.75 & 0.67 & 74.5 & 8.68 & D.abyssinica:Nigeria & SRR8451379 \\
\hline ns019_A52 & A52 & 1.66 & 1.63 & 1.44 & 0.02 & 70.8 & 3.52 & D.abyssinica:Benin & SRR8451376 \\
\hline ns020_A62 & A62 & 2.35 & 2.31 & 2.06 & 0.02 & 77.3 & 4.60 & D.abyssinica:Benin & SRR8451375 \\
\hline ns021_A67 & A67 & 7.54 & 7.42 & 6.12 & 0.12 & 85.2 & 12.40 & D.abyssinica:Benin & SRR8451343 \\
\hline ns023_A467 & A467 & 5.72 & 5.64 & 5.08 & 0.06 & 82.0 & 10.69 & D.abyssinica:Benin & SRR8451345 \\
\hline ns024_A537 & A537 & 6.22 & 6.13 & 5.28 & 0.05 & 79.3 & 11.49 & D.abyssinica:Benin & SRR8451346 \\
\hline ns025_A3009 & А3009 & 3.33 & 3.27 & 2.92 & 0.03 & 76.7 & 6.57 & D.abyssinica:Benin & SRR8451347 \\
\hline ns027_A5068 & A5068 & 1.98 & 1.95 & 1.67 & 0.04 & 65.7 & 4.38 & D.abyssinica:Ghana & SRR8451349 \\
\hline ns028_A5045 & A5045 & 2.61 & 2.56 & 2.21 & 0.04 & 74.4 & 5.12 & D.abyssinica:Ghana & SRR8451350 \\
\hline ns029_A5047 & A5047 & 3.32 & 3.27 & 2.80 & 0.04 & 75.0 & 6.46 & D.abyssinica:Ghana & SRR8451351 \\
\hline ns030_A5048 & A5048 & 9.39 & 9.23 & 7.75 & 0.10 & 82.9 & 16.14 & D.abyssinica:Ghana & SRR8451352 \\
\hline ns031_A5059 & A5059 & 10.28 & 10.10 & 7.09 & 1.66 & 82.5 & 14.82 & D.abyssinica:Ghana & SRR8451320 \\
\hline ns032_A5061 & A5061 & 2.81 & 2.77 & 1.91 & 0.54 & 72.4 & 4.55 & D.abyssinica:Ghana & SRR8451319 \\
\hline ns033_A5066 & A5066 & 8.09 & 7.95 & 6.74 & 0.11 & 80.7 & 14.41 & D.abyssinica:Ghana & SRR8451318 \\
\hline ns034_A5067 & A5067 & 7.67 & 7.55 & 6.51 & 0.06 & 82.0 & 13.71 & D.abyssinica:Ghana & SRR8451317 \\
\hline ns035_P5344 & P5344 & 3.33 & 3.30 & 2.46 & 0.10 & 70.6 & 6.02 & D.praehensilis:Cameroon:Cameroonian D.praehensilis & SRR8451316 \\
\hline ns036_P5350 & P5350 & 4.06 & 4.02 & 2.77 & 0.20 & 63.5 & 7.52 & D.praehensilis:Cameroon:Cameroonian D.praehensilis & SRR8451315 \\
\hline ns037_P5358 & P5358 & 4.21 & 4.17 & 3.09 & 0.15 & 73.2 & 7.29 & D.praehensilis:Cameroon:Cameroonian D.praehensilis & SRR8451314 \\
\hline ns038_P5369 & P5369 & 3.10 & 3.08 & 2.17 & 0.32 & 70.2 & 5.34 & D.praehensilis:Cameroon:Cameroonian D.praehensilis & SRR8451313 \\
\hline ns039_P5378 & P5378 & 3.01 & 2.99 & 2.31 & 0.05 & 70.5 & 5.66 & D.praehensilis:Cameroon:Cameroonian D.praehensilis & SRR8451322 \\
\hline ns040_P5381 & P5381 & 3.90 & 3.87 & 2.97 & 0.11 & 72.8 & 7.05 & D.praehensilis:Cameroon:Cameroonian D.praehensilis & SRR8451321 \\
\hline
\end{tabular}




\begin{tabular}{|c|c|c|c|c|c|c|c|c|c|}
\hline ns041_P5404 & P5404 & 4.53 & 4.49 & 3.31 & 0.31 & 74.3 & 7.69 & D.praehensilis:Cameroon:Cameroonian D.praehensilis & SRR8451462 \\
\hline ns042_P5413 & P5413 & 3.78 & 3.75 & 2.82 & 0.16 & 73.5 & 6.62 & D.praehensilis:Cameroon:Cameroonian D.praehensilis & SRR8451463 \\
\hline ns043_P5417 & P5417 & 4.61 & 4.58 & 3.44 & 0.19 & 74.1 & 8.01 & D.praehensilis:Cameroon:Cameroonian D.praehensilis & SRR8451460 \\
\hline ns044_P5420 & P5420 & 2.25 & 2.23 & 1.65 & 0.15 & 65.9 & 4.31 & D.praehensilis:Cameroon:Cameroonian D.praehensilis & SRR8451461 \\
\hline ns045_P5424 & P5424 & 5.30 & 5.26 & 3.74 & 0.42 & 74.4 & 8.68 & D.praehensilis:Cameroon:Cameroonian D.praehensilis & SRR8451466 \\
\hline ns046_P5427 & P5427 & 4.25 & 4.22 & 3.24 & 0.05 & 72.9 & 7.66 & D.praehensilis:Cameroon:Cameroonian D.praehensilis & SRR8451467 \\
\hline ns047_P5430 & P5430 & 3.34 & 3.31 & 2.41 & 0.10 & 63.5 & 6.56 & D.praehensilis:Cameroon:Cameroonian D.praehensilis & SRR8451464 \\
\hline ns048_P5434 & P5434 & 2.80 & 2.77 & 2.10 & 0.06 & 61.8 & 5.86 & D.praehensilis:Cameroon:Cameroonian D.praehensilis & SRR8451465 \\
\hline ns049_P5438 & P5438 & 3.64 & 3.61 & 2.36 & 0.62 & 70.6 & 5.76 & D.praehensilis:Cameroon:Cameroonian D.praehensilis & SRR8451468 \\
\hline ns051_P5448 & P5448 & 4.73 & 4.69 & 3.66 & 0.09 & 73.6 & 8.58 & D.praehensilis:Cameroon:Cameroonian D.praehensilis & SRR8451449 \\
\hline ns054_P5318 & P5318 & 5.04 & 4.99 & 3.07 & 0.62 & 67.7 & 7.83 & D.praehensilis:Cameroon:Cameroonian D.praehensilis & SRR8451450 \\
\hline ns055_P5746 & P5746 & 3.80 & 3.77 & 2.66 & 0.43 & 65.3 & 7.02 & D.praehensilis:Nigeria:Western D.praehensilis & SRR8451453 \\
\hline ns056_P5708 & P5708 & 6.19 & 6.13 & 4.22 & 0.39 & 64.5 & 11.30 & D.praehensilis:Nigeria:Western D.praehensilis & SRR8451452 \\
\hline ns057_P5710 & P5710 & 3.89 & 3.86 & 2.61 & 0.48 & 70.0 & 6.42 & D.praehensilis:Nigeria:Western D.praehensilis & SRR8451455 \\
\hline ns058_P5713 & P5713 & 3.24 & 3.21 & 2.34 & 0.22 & 67.2 & 6.02 & D.praehensilis:Nigeria:Western D.praehensilis & SRR8451454 \\
\hline ns059_P5716 & P5716 & 2.56 & 2.53 & 1.91 & 0.03 & 63.0 & 5.23 & D.praehensilis:Nigeria:Western D.praehensilis & SRR8451457 \\
\hline ns061_P5720 & P5720 & 3.87 & 3.84 & 2.99 & 0.17 & 73.5 & 7.02 & D.praehensilis:Nigeria:Western D.praehensilis & SRR8451430 \\
\hline ns064_P5729 & P5729 & 7.31 & 7.25 & 4.58 & 1.01 & 72.5 & 10.89 & D.praehensilis:Nigeria:Western D.praehensilis & SRR8451433 \\
\hline ns065_P424 & P424 & 3.46 & 3.40 & 3.03 & 0.04 & 79.1 & 6.61 & D.praehensilis:Benin:Western D.praehensilis & SRR8451426 \\
\hline ns066_P425 & P425 & 1.63 & 1.60 & 1.44 & 0.02 & 69.5 & 3.57 & D.praehensilis:Benin:Western D.praehensilis & SRR8451427 \\
\hline ns067_P457 & P457 & 4.21 & 4.13 & 3.46 & 0.12 & 74.5 & 8.01 & D.praehensilis:Benin:Western D.praehensilis & SRR8451428 \\
\hline ns068_P462 & P462 & 4.33 & 4.26 & 3.68 & 0.08 & 79.7 & 7.98 & D.praehensilis:Benin:Western D.praehensilis & SRR8451429 \\
\hline ns069_P323 & P323 & 4.22 & 4.15 & 3.70 & 0.05 & 80.5 & 7.94 & D.praehensilis:Benin:Western D.praehensilis & SRR8451435 \\
\hline ns070_P464 & P464 & 5.29 & 5.21 & 4.65 & 0.05 & 80.6 & 9.96 & D.praehensilis:Benin:Western D.praehensilis & SRR8451436 \\
\hline ns073_P2990 & P2990 & 2.88 & 2.84 & 2.56 & 0.03 & 77.6 & 5.70 & D.praehensilis:Benin:Western D.praehensilis & SRR8451409 \\
\hline ns075_P4918 & P4918 & 2.45 & 2.40 & 1.82 & 0.27 & 72.6 & 4.33 & D.praehensilis:Ghana:Western D.praehensilis & SRR8451415 \\
\hline ns076_P4919 & P4919 & 5.46 & 5.36 & 4.04 & 0.45 & 79.4 & 8.79 & D.praehensilis:Ghana:Western D.praehensilis & SRR8451414 \\
\hline ns077_P4920 & P4920 & 6.04 & 5.93 & 4.63 & 0.53 & 80.3 & 9.95 & D.praehensilis:Ghana:Western D.praehensilis & SRR8451413 \\
\hline ns078_P4921 & P4921 & 4.73 & 4.65 & 3.73 & 0.31 & 79.5 & 8.11 & D.praehensilis:Ghana:Western D.praehensilis & SRR8451412 \\
\hline ns079_P4928 & P4928 & 3.77 & 3.71 & 2.99 & 0.24 & 78.4 & 6.57 & D.praehensilis:Ghana :Western D.praehensilis & SRR8451407 \\
\hline
\end{tabular}

